\title{
EXTENSIONS OF HARDY SPACES AND THEIR USE IN ANALYSIS
}

\author{
BY RONALD R. COIFMAN AND GUIDO WEISS ${ }^{1}$
}

1. Introduction. It is well known that the theory of functions plays an important role in the classical theory of Fourier series. Because of this certain function spaces, the $H^{p}$ spaces, have been studied extensively in harmonic analysis. When $p>1, L^{p}$ and $H^{p}$ are essentially the same; however, when $p \leqslant 1$ the space $H^{p}$ is much better adapted to problems arising in the theory of Fourier series. We shall examine some of the properties of $H^{p}$ for $p \leqslant 1$ and describe ways in which these spaces have been characterized recently. These characterizations enable us to extend their definition to a very general setting that will allow us to unify the study of many extensions of classical harmonic analysis.

The theory of $H^{p}$ spaces on $\mathbf{R}^{n}$ has recently received an important impetus from the work of C. Fefferman and E. M. Stein [29]. Their work resulted in many applications involving sharp estimates for convolution operators. It is not immediately apparent how much of a role the differential structure of $\mathbf{R}^{n}$ plays in obtaining these results. Our purpose is to isolate from this theory some of the measure theoretic and geometric properties that enable us to obtain in a unified form many of these applications as well as other results in harmonic analysis. We shall not deal with those questions involving $H^{p}$ spaces that are not relevant to our purpose. Some general references involving harmonic analysis and $H^{p}$ spaces are [23], [64], [27], [62], [57] and [55].

The main tool in our development is an extension and a refinement of the Calderón-Zygmund decomposition of a function into a "good" and "bad" part. This tool is presented in the proof of Theorem A and is of a somewhat technical nature. It is included here in order to make the presentation of the theory we develop essentially self-contained. In some examples we give applications of this theory that require material not presented here. We do, however, give the necessary references. In this sense, we hope that this exposition is accessible to a general audience.

Before beginning our presentation we would like to thank our colleagues A. Baernstein, Y. Meyer, R. Rochberg and E. M. Stein who read a large part of this manuscript and made many useful suggestions.

Suppose $f$ is a real-valued integrable function on $T$, the perimeter of the unit disc in the plane (which we identify in the usual way with $[-\pi, \pi)$ ). Suppose $f$

\footnotetext{
${ }^{1}$ This paper is based on the material presented by the last-named author in an Invited Address to the American Mathematical Society meeting in Saint Louis on April 11-12, 1975. This lecture, in turn, was based on results obtained in a collaboration by the two authors of this paper; received by the editors January $20,1976$.

AMS (MOS) subject classifications (1970). Primary 30A78, 42A18, 42A40; Secondary 32A07, 42A56, $43 \mathrm{~A} 85$.
} 
has the Fourier series $\sum_{-\infty}^{\infty} c_{k} e^{i k \theta}$. Let $F$ be the analytic function defined by the power series $c_{0}+2 \sum_{1}^{\infty} c_{k} z^{k},|z|<1$. This association of analytic functions in the disc $D=\{z \in \mathbf{C}:|z|<1\}$ with real functions on $T$ is the basis of a general technique, known as the use of complex methods, for obtaining results in the theory of Fourier series. The essential ingredients of this procedure can be described briefly in the following way. Certain basic operations on functions $f$ on $T$ (often realized as multiplication of the Fourier coefficients of $f$ by specific sequences) can be interpreted as operations on the associated analytic functions $F$. Powerful tools from the theory of functions (such as factorization of zeroes, conformal mappings, etc.) can be used to study the properties of such operations on the functions $F$. The properties can then be translated as results about functions on $T$ since $f$ can be recovered from $F$ by taking the almost everywhere existing radial limits

$$
f(\theta)=\lim _{r \rightarrow 1} \operatorname{Re}\left\{F\left(r e^{i \theta}\right)\right\}^{2}
$$

If $f \in L^{p}(T), 1<p<\infty$, the M. Riesz inequality (see [64] for a proof) is satisfied by the analytic function $F$ and the original function $f$ :

$$
\left(\int_{-\pi}^{\pi}\left|F\left(r e^{i \theta}\right)\right|^{p} d \theta\right)^{1 / p} \leqslant A_{p}\|f\|_{p}
$$

where $A_{p}$ is independent of $f$ and $r, 0 \leqslant r<1$. Moreover, the radial limits (1.1) can be taken in the $L^{p}$ norm as well.

In 1915 [33] Hardy considered the means

$$
\mu_{p}(F ; r)=\left(\int_{-\pi}^{\pi}\left|F\left(r e^{i \theta}\right)\right|^{p} d \theta\right)^{1 / p},
$$

$0<p<\infty$, as functions of $r$ and showed that they behave similarly to the "maximum modulus" $\mu_{\infty}(F ; r)=\max \left\{\left|F\left(r e^{i \theta}\right)\right| ; \theta \in[-\pi, \pi)\right\}$ (among other things, he showed that $\mu_{p}(F ; r)$ increases with $r$ and satisfies the conclusion of the "three circle theorem"). In $1923 \mathrm{~F}$. Riesz (see [48]) introduced the class of functions $F$, analytic in $D$, for which

$$
\|F\|_{H^{p}}=\sup _{0 \leqslant r<1} \mu_{p}(F ; r)<\infty,
$$

where $0<p \leqslant \infty$, and denoted this class by the symbol $H^{p}$ in obvious homage to Hardy. Since that time these spaces have become known as Hardy spaces. ${ }^{3}$

\footnotetext{
2 Many examples of this technique can be found in the books of Duren [23], Hoffman [35] and Zygmund [64].

${ }^{3}$ In his 1923 paper F. Riesz established many of the important properties of these spaces (e.g. the Blaschke product factorization, the existence of boundary values and other results). His brother M. Riesz also made important contributions in this field. Thus, it could be argued fairly that the name "Riesz" should also be attached to these spaces. We shall not enter into such an argument but would also like to point out that subsequent contributions by Hardy and Littlewood were most basic to the development of the theory of $H^{p}$ spaces.
} 
For the next few pages we shall assume that $L^{p}(T)$ consists only of realvalued functions and, thus, is a vector space over the real numbers. Though not essential, this assumption will simplify some of the assertions we are going to make.

An immediate consequence of the M. Riesz inequality is that $F$ $\in H^{p}$ whenever $f \in L^{p}(T), 1<p<\infty$. Conversely, if an analytic function $F$ belongs to $H^{p}$ then the limit function $f$ in (1.1) exists (in the $L^{p}$ norm and a.e.); moreover, $F$ can be reconstructed from $f$ (up to an imaginary constant) in the manner described above, provided $1<p<\infty$ (see [64, Chapter VII]). Hence, for these values of $p, L^{p}(T)$ is the space $\Re H^{p}$ consisting of the boundary values of the real parts of functions in $H^{p}$ (in the sense of (1.1)). ${ }^{4}$ The situation is completely different when $p=1$. In fact, for a nonnegative $f \in L^{1}(T), F \in H^{1}$ if and only if $\int_{-\pi}^{\pi} f\left(1+\log ^{+} f\right)<\infty$ (see Chapter VII in [64]). Characterizations of $\Re e H^{1}$ have been obtained only recently; in a sense, this is the origin of this exposition. We shall explain these characterizations in great detail later.

It was observed by Hardy and Littlewood, as well as many others, that there are many results in Fourier analysis that hold for $L^{p}(T), 1<p<\infty$, fail to be true for $L^{1}(T)$ and, yet, remain true for $\operatorname{Re} H^{1}$. Since $L^{q}(T) \varsubsetneqq L^{p}(T)$ $\varsubsetneqq \Re H^{1} \varsubsetneqq L^{1}(T)$ when $1<p<q \leqslant \infty$ we see that $\operatorname{Re} H^{1}$ can be regarded as a good "substitute" for $L^{1}(T)$ that is endowed with many of the properties enjoyed by the $L^{p}$ spaces for $1<p<\infty$ and, moreover, contains all these spaces. Let us examine some specific examples of this situation.

Suppose $f \in L^{p}(T), 1<p \leqslant 2$, has the Fourier series $\sum_{-\infty}^{\infty} c_{k} e^{i k \theta}$. A wellknown result of Paley (see [64, Vol. 2. p. 111]) asserts, in part, that

$$
\sum_{-\infty}^{\infty}\left|c_{k}\right|^{p}|k|^{p-2}<\infty .^{5}
$$

This result is false when $p=1$. In fact, there exists an integrable function whose Fourier series is $\sum_{2}^{\infty} \cos k \theta / \log k$ (see [64, Vol. 1, p. 184]). On the other hand, Hardy has shown that if $f \in G R H^{1}$ we have

$$
\sum_{-\infty}^{\infty} \frac{\left|c_{k}\right|}{|k|}<\infty .
$$

This last inequality is more striking when we realize that no condition on the rate of convergence to 0 can be given for the Fourier coefficients of a function in $L^{1}(T)$ (see [64, Vol. 1, p. 183]); in this sense, the RiemannLebesgue theorem, asserting that these coefficients tend to zero, cannot be improved. Another example of this phenomenon is Paley's result:

$$
\sum_{-\infty}^{\infty}\left|c_{2^{k}}\right|^{2}<\infty
$$

${ }^{4}$ It is not hard to show, making use of (1.2), that the space of complex-valued $L^{p}(T)$ functions and $H^{p}$ (with norm given by (1.3)) are equivalent Banach spaces when $1<p<\infty$ (see Boas [1]).

$5 \mathrm{We}$ are adopting the standard notation: $\Sigma^{\prime}$ means that we are summing over all nonzero integers $k$ : 
is satisfied by the Fourier coefficients of a function $f$ in $R e H^{1}$ but does not hold for an arbitrary function in $L^{1}(T)$.

Perhaps the most basic difference between $L^{1}(T)$ and $\Re e H^{1}$ that was discovered by Hardy and Littlewood involves the Poisson integral of $f$ :

$$
\left(P_{r} * f\right)(\theta)=\frac{1}{2 \pi} \int_{-\pi}^{\pi} \frac{1-r^{2}}{1-2 r \cos (\theta-\psi)+r^{2}} f(\psi) d \psi \equiv u_{f}\left(r e^{i \theta}\right),
$$

$0 \leqslant r<1$. Let

$$
\left(P^{*} f\right)(\theta)=\sup _{\left|w-e^{i \theta}\right| \leqslant 2(1-|w|)}\left|u_{f}(w)\right|
$$

denote the (nontangential) maximal operator associated with this integral. Hardy and Littlewood showed that $P^{*} f \in L^{1}(T)$ when $f \in R e H^{1}$, while this is not true for a general $f \in L^{1}(T)$. It has been understood for a long time that this result represents a basic difference between these two function spaces; however, it was only recently shown by Burkholder, Gundy and Silverstein [2] that we have here a real-variable characterization of $\Re$ e $H^{1}:$ for $f \in L^{1}(T)$, $P^{*} f \in L^{1}(T)$ if and only if $f \in \Re e H^{1}{ }^{6}$

If $f \in \Re e H^{1}$ then, by definition, $f(\theta)=\Re\left(e^{i \theta}\right)$, where $F \in H^{1}$. If we make the restriction that the imaginary part of $F$ vanishes at 0 , the function $F$ is unique. We can then put a norm on $\Re$ e $H^{1}$ by letting $\|f\|_{H^{1}} \equiv\|F\|_{H^{1}}$ (see (1.3)). With this norm $\Re$ e $H^{1}$ is a Banach space.

The next important step toward an understanding of $\Re$ e $H^{1}$ was taken by $C$. Fefferman who characterized the dual, $\left\{\operatorname{Re} H^{1}\right\}^{*}$, of this space as the class of functions of Bounded Mean Oscillation (BMO) originally introduced by John and Nirenberg [38]: a periodic function $l$ (of period $2 \pi$ ) is said to be in the class $B M O$ provided it belongs to $L^{1}(T)$ and

$$
\int_{I}\left|l(\theta)-m_{I}(l)\right| \frac{d \theta}{|I|} \leqslant c
$$

for all finite intervals $I$, where $c$ is independent of $I$ and $m_{I}(l)$ $=(1 /|I|) \int_{I} l(\theta) d \theta$. The space $B M O$ is a Banach space if we let

$$
\|l\|_{B M O}=\left|\frac{1}{2 \pi} \int_{0}^{2 \pi} l\right|+\sup _{I} \frac{1}{|I|} \int_{I}\left|l-m_{I}(l)\right|
$$

be its norm. C. Fefferman showed that the mappings

$$
f \rightarrow \int_{-\pi}^{\pi} f(\theta) l(\theta) d \theta,
$$

induced by the various $l \in B M O$, yield all the bounded linear functionals on $\Re H^{1}$ and their norms as linear functionals are equivalent to $\|\cdot\|_{B M O}$. (The

6 We mentioned before that, for $f \geqslant 0$, being a member of $\operatorname{Re} H^{1}$ is equivalent to the integrability of $f \log ^{+} f$. On the other hand, this last condition is equivalent to the integrability of $P^{*} f$ (in this connection, and for higher dimensional extensions, see E. M. Stein [53]). 
integrals $\int_{-\pi}^{\pi} f l$ have to be interpreted appropriately; we shall consider this point later in $\$ 3.)^{7}$

This characterization of $\left\{\operatorname{Re} H^{1}\right\}^{*}$ is easily seen to be equivalent to the following description of the functions belonging to $R e H^{1}$. We begin by introducing a basic "building block" for the elements of $\Re$ e $H^{1}$ :

Definition. An atom (more precisely, a 1-atom) is either the function $a_{0}(\theta) \equiv 1 / 2 \pi$ or a function $a(\theta),-\pi \leqslant \theta<\pi$, satisfying:

(i) The support of $a$ is contained in an interval $I \subset[-\pi, \pi){ }^{8}$

(ii) $|a(\theta)| \leqslant|I|^{-1}$ for all $\theta \in[-\pi, \pi)$;

(iii) $\int_{0}^{2 \pi} a(\theta) d \theta=0$.

The above-mentioned characterization is, then, simply: a function $f$ belongs to $\operatorname{Re} H^{1}$ if and only if

$$
f=\sum_{j=0}^{\infty} \lambda_{j} a_{j}
$$

where $a_{j}$ is an atom, $0 \leqslant j$, and $\sum_{j=0}^{\infty}\left|\lambda_{j}\right|<\infty$. Moreover, if $F$ is the analytic function corresponding to $f$ (in the manner described above) there exist absolute constants $c_{1}$ and $c_{2}$ such that

$$
c_{1}\|F\|_{H^{1}} \leqslant \inf \sum_{0}^{\infty}\left|\lambda_{j}\right| \leqslant c_{2}\|F\|_{H^{1}},
$$

where the infimum is taken over all decompositions (1.7).

This characterization of $\operatorname{Re} H^{1}$ leads us to a corresponding real-variable characterization of $\operatorname{Re} H^{p}, 0<p \leqslant 1$. The example

$$
F(z)=(1 / 2 \pi)[(1+z) /(1-z)]
$$

of a function in $H^{p}, 0<p<1$, whose real part converges to 0 as $z=r e^{i \theta} \rightarrow e^{i \theta}($ as $r \rightarrow 1)$ for $\theta \in(0,2 \pi)$, indicates that $\Re H^{p}$ is not a function space; rather, it should be defined as a space of distributions (in this example, the boundary value is the Dirac measure concentrated at $\theta=0$ ). When $1 / 2<p \leqslant 1$ these distributions are obtained by a simple renormalization of the atoms we have just introduced: We call a function $a(\theta)$ a $p$-atom if it satisfies properties (i) and (iii) and, instead of (ii), if it satisfies $|a(\theta)|$ $\leqslant|I|^{-1 / p}$.

The characterization of $\operatorname{Re} H^{p}$ can be stated as follows (see [10]):

THEOREM I. A distribution $f$ belongs to $\operatorname{Re} H^{p}, 0<p \leqslant 1$, if and only if it can be represented in the form $f=\sum_{j=0}^{\infty} \lambda_{j} a_{j}$, where $a_{j}$ is a p-atom, $0 \leqslant j$, and $\sum_{0}^{\infty}\left|\lambda_{j}\right|^{p}<\infty$.

It is a common practice of modern mathematics to transform a theorem characterizing a mathematical notion into a definition of that notion. Doing

\footnotetext{
7 This will become evident later on when we discuss the more general setting for the theory of $H^{p}$ spaces.

8 We are really dealing with functions defined on the perimeter of the unit circle and our "intervals" are intervals in this perimeter. Thus, we allow $I$ to have the form $\{\theta:-\pi \leqslant \theta<a$ or $b$ $<\theta \leqslant \pi$, where $a \leqslant b$.

${ }^{9}$ For the cases $0<p<1 / 2$ we also have to modify condition (iii). We shall discuss this matter later.
} 
this is particularly fruitful if the characterization has three basic properties:

(a) Known results follow easily from it.

(b) New results follow easily from it.

(c) The notion being characterized can be extended to new situations and this leads to a theory as useful and powerful as the original one.

Theorem I has these features. First of all, it is clear that the defining properties of an atom make sense in any topological measure space where a basis for open sets (such as the intervals used above) is prescribed. This is one of the major points of this presentation. First, however, we shall give some illustrations of properties (a) and (b).

We begin by establishing Hardy's inequality (1.5). Let us first observe that it suffices to prove (1.5) for the Fourier coefficients of an atom. In fact, we shall show that

$$
\sum_{k=-\infty}^{\infty} \frac{\left|a_{k}\right|}{|k|} \leqslant 4
$$

whenever $a_{k}=(1 / 2 \pi) \int_{-\pi}^{\pi} a(\theta) e^{-i k \theta} d \theta$ are the Fourier coefficients of a 1-atom $a(\theta)$. Hardy's inequality then follows immediately from (1.9) and the representation (1.7) of functions in $\operatorname{Re} H^{1} \cdot{ }^{10}$ Thus, let $a(\theta)$ be a 1 -atom whose supporting interval, $I$, in conditions (i) and (ii) is centered at 0 (there is no loss in generality since $\left|a_{k}\right|$ is unchanged by translations). Using first (iii) and then (ii) we have

$$
\begin{aligned}
\left|a_{k}\right| & =\left|\frac{1}{2 \pi} \int_{I} a(\theta)\left[e^{-i k \theta}-1\right] d \theta\right| \leqslant \frac{1}{2 \pi} \int_{I}|a(\theta)||k \theta| d \theta \\
& \leqslant \frac{|k||I|}{2 \pi} \int_{I}|I|^{-1} d \theta=\frac{|k||I|}{2 \pi} .
\end{aligned}
$$

Moreover, again using (ii) we have

$$
\sum_{k=-\infty}^{\infty}\left|a_{k}\right|^{2}=\frac{1}{2 \pi} \int_{-\pi}^{\pi}|a(\theta)|^{2} d \theta \leqslant \frac{1}{2 \pi} \int_{I}|I|^{-2} d \theta=\frac{1}{2 \pi}|I|^{-1} .
$$

Making use of these two estimates we have

$$
\begin{aligned}
\sum_{k=-\infty}^{\infty} \frac{\left|a_{k}\right|}{|k|} & =\sum_{|k| \leqslant|I|^{-1}} \frac{\left|a_{k}\right|}{|k|}+\sum_{|k|>|I|^{-1}} \frac{\left|a_{k}\right|}{|k|} \\
& \leqslant \frac{1}{\pi} \sum_{|k| \leqslant|I|^{-1}}|I|+\left(\sum_{-\infty}^{\infty}\left|a_{k}\right|^{2}\right)^{1 / 2}\left(\sum_{|k|>|I|^{-1}} k^{-2}\right)^{1 / 2} \leqslant 4
\end{aligned}
$$

and (1.9) is established. In view of (1.8) we obtain

10 This is an important feature of the use of atoms: many problems can be reduced to considerations involving only atoms and, because of this, the resolution of these problems is made simpler. 


$$
\sum_{-\infty}^{\infty}, \frac{\left|a_{k}\right|}{|k|} \leqslant 4 c_{2}\|F\|_{H^{1}}
$$

for any $F \in \operatorname{Re} H^{1}$.

Our next observation is that we can easily identify the dual spaces of gre $H^{p}, 0<p \leqslant 1$, if we know Theorem I. We restrict our attention to the case $1 / 2<p \leqslant 1$, since we have defined a $p$-atom only for this range of $p$. If $L \in\left\{\operatorname{Re} H^{p}\right\}^{*}$ it is easy to show (and we will do so later in a more general context) that the action of this linear functional on atoms $a(\theta)$ is given by an integrable function $l(\theta)$ as follows:

$$
L a=\int_{-\pi}^{\pi} a(\theta) l(\theta) d \theta .
$$

Moreover, the continuity of $L$ is equivalent to the "boundedness" $|L a| \leqslant\|L\|$. We claim that $l$ satisfies

$$
\sup _{I \subset[-\pi, \pi)}|I|^{-1 / p} \int_{I}\left|l(\theta)-m_{I}(l)\right| d(\theta) \leqslant 2\|L\| .
$$

To see this we choose a function $f$ supported in $I$ such that $\|f\|_{\infty} \leqslant 1$. Obviously, if $\chi_{I}$ is the characteristic function of $I$,

$$
a(\theta)=2^{-1}|I|^{-1 / p}\left\{f(\theta)-m_{I}(f) \chi_{I}(\theta)\right\}
$$

is a $p$-atom. We thus have

$$
\begin{aligned}
\left|\int_{I} f(\theta)\left[l(\theta)-m_{I}(l)\right] d \theta\right| & =\left|\int_{I}\left[f(\theta)-m_{I}(f)\right] l(\theta) d \theta\right| \\
& =2|I|^{1 / p}\left|\int_{I} a(\theta) l(\theta) d \theta\right| \leqslant 2|I|^{1 / p}\|L\| .
\end{aligned}
$$

Inequality (1.11) now follows by taking the supremum over all such functions $f$. It is also clear, by retracing our steps, that if the supremum in (1.11) is finite then the integral above this inequality defines a bounded linear functional on Re $H^{p}$.

When $p=1(1.11)$ is the defining condition for the space $B M O$. This condition has been studied extensively for $p<1$ as well (see [7] and [46]). In particular, as we shall show later, when $1 / 2<p<1(1.11)$ is equivalent to

$$
|l(\theta)-l(\psi)| \leqslant c|\theta-\psi|^{\alpha}, \quad \alpha=1 / p-1 .
$$

This yields the characterization of the dual of $\Re e H^{p}$ given by Duren, Romberg and Shields [24]. At this point it is relevant to point out that results involving $H^{p}$ spaces are translated by duality into results on Lipschitz spaces (i.e. spaces of functions satisfying inequalities of the type (1.11) or (1.12)). We shall return to this point after we have introduced our extensions of the Hardy spaces.

We have encountered many other situations where Theorem I can be used directly and efficiently to produce known results in the theory of $H^{p}$ spaces. Let us now illustrate the fact that Theorem I enjoys property (b); that is, new 
results can be obtained from it. Perhaps the most striking development in this direction is that we can obtain a simple and useful characterization of the Fourier coefficients of elements in $\operatorname{Re} H^{p}$. It is well known that such a characterization exists for $L^{2}(T)$ via the Plancherel theorem; that is, a necessary and sufficient condition for a sequence $\left\{a_{k}\right\}, k=0, \pm 1, \pm 2, \ldots$, to be the sequence of Fourier coefficients of an $f \in L^{2}(T)$ is $\sum_{k=-\infty}^{\infty}\left|a_{k}\right|^{2}<\infty$. This condition enables us to easily characterize those linear operators commuting with translations that are bounded on $L^{2}(T)$. In fact, it is easy to see that if $A$ is such an operator then its action is determined by an associated sequence $\left\{\mu_{k}\right\}$ in such a way that if $f$ has Fourier coefficients $\left\{a_{k}\right\}$ then $A f$ has Fourier coefficients $\left\{\mu_{k} a_{k}\right\}{ }^{11}$ It is immediate to check that $\left\{\mu_{k} a_{k}\right\}$ is square summable for all square summable sequences $\left\{a_{k}\right\}$ if and only if $\left\{\mu_{k}\right\}$ is bounded. The study of convolution operators on $\Re \mathrm{e} H^{p}$ is not quite so simple; however, we shall see that the characterization we will give of the Fourier coefficients of the elements of these spaces is very useful for such a study.

We first observe that, if $a(\theta)$ is a real-valued (nonconstant) 1-atom whose supporting interval is centered at 0 , then, making use of (i), (ii) and the estimate $\left|e^{i \theta}-1\right|^{2} \leqslant|\theta|^{2}$,

$$
\left(\int_{-\pi}^{\pi}|a(\theta)|^{2} d \theta\right)^{1 / 2}\left(\int_{-\pi}^{\pi}|a(\theta)|^{2}\left|e^{i \theta}-1\right|^{2} d \theta\right)^{1 / 2} \leqslant \frac{1}{\sqrt{2}} .
$$

Let $\sum_{-\infty}^{\infty} a_{k} e^{i k \theta}$ be the Fourier series of $a(\theta)$ and $A(z)=2 \Sigma_{1}^{\infty} a_{k} z^{k}$ the analytic function associated with $a(\theta)$ described in the beginning of this exposition. It is a well-known fact (see Zygmund's book [64]) that the boundary values $A\left(e^{i \theta}\right)=\lim _{r \rightarrow 1} A\left(r e^{i \theta}\right)$ cannot vanish on a set of positive measure. Thus, $A\left(e^{i \theta}\right)$ could be an atom, or a multiple of one, only if it were bounded by $1 / 2 \pi$; but simple examples (we can choose $a(\theta)=(2 \pi)^{-1} \operatorname{sgn} \theta$, $-\pi \leqslant \theta<\pi)$ show that this cannot be expected. Nevertheless, $A\left(e^{i \theta}\right)$ does inherit the very basic property (1.13). In fact, using Plancherel's theorem and the fact that $a_{-k}=\bar{a}_{k}$ we have

$$
\begin{aligned}
\left(\int_{-\pi}^{\pi}\left|A\left(e^{i \theta}\right)\right|^{2} d \theta\right)^{1 / 2} & \left(\int_{-\pi}^{\pi}\left|A\left(e^{i \theta}\right)\right|^{2}\left|1-e^{i \theta}\right|^{2} d \theta\right)^{1 / 2} \\
& =8 \pi\left(\sum_{k=1}^{\infty}\left|a_{k}\right|^{2}\right)^{1 / 2}\left(\sum_{k=1}^{\infty}\left|a_{k+1}-a_{k}\right|^{2}+\left|a_{1}\right|^{2}\right)^{1 / 2} \\
& =4 \pi\left(\sum_{k=-\infty}^{\infty}\left|a_{k}\right|^{2}\right)^{1 / 2}\left(\sum_{k=-\infty}^{\infty}\left|a_{k+1}-a_{k}\right|^{2}\right)^{1 / 2} \\
& =\left(\int_{-\pi}^{\pi}|a(\theta)|^{2} d \theta\right)^{1 / 2}\left(\int_{-\pi}^{\pi}|a(\theta)|^{2}\left|1-e^{i \theta}\right|^{2} d \theta\right)^{1 / 2} .
\end{aligned}
$$

\footnotetext{
11 The property of commuting with translations easily implies that the functions $e^{i k \theta}, k=0$, $\pm 1, \pm 2, \ldots$, are proper vectors for the operator $A$. The sequence $\left\{\mu_{k}\right\}$ is the corresponding sequence of proper values of $A$. Such operators are called multiplier operators or (more appropriately) convolution operators; $\left\{\mu_{k}\right\}$ is referred to as the sequence of multipliers.
} 
More generally, let us consider (possibly complex-valued) functions $M$ $\in L^{2}(T)$ satisfying

$$
\begin{gathered}
\left(\int_{-\pi}^{\pi}|M(\theta)|^{2} d \theta\right)^{1 / 4}\left(\int_{-\pi}^{\pi}|M(\theta)|^{2}\left|e^{i \theta}-1\right|^{2} d \theta\right)^{1 / 4} \leqslant 1, \\
\int_{-\pi}^{\pi} M(\theta) d \theta=0 .
\end{gathered}
$$

Such functions can be shown to be sums of atoms centered at 0 that are "neatly stacked one on top of the other". We shall return to this point later; we mention this now, however, to justify calling such functions molecules. ${ }^{12}$ It turns out that molecules are practically as basic as atoms in the study of $H^{p_{\text {. }}}$ space theory.

We first observe for any $M$ in $L^{2}(T)$

$$
\int_{-\pi}^{\pi}|M(\theta)| d \theta \leqslant 3 \pi\left(\int_{-\pi}^{\pi}|M(\theta)|^{2} d \theta\right)^{1 / 4}\left(\int_{-\pi}^{\pi}|M(\theta)|^{2}\left|e^{i \theta}-1\right|^{2} d \theta\right)^{1 / 4}
$$

In fact, let $M_{0}(\theta)=\left|1-e^{i \theta}\right| M(\theta)$; then

$$
\begin{aligned}
\int_{-\pi}^{\pi}|M(\theta)| d \theta & =\left(\int_{|\theta| \leqslant A^{2} / 2}+\int_{|\theta|>A^{2} / 2}\right)|M(\theta)| d \theta \\
\leqslant & \|M\|_{2}\left(2 \int_{0}^{A^{2} / 2} d \theta\right)^{1 / 2} \\
& +\left(\int_{-\pi}^{\pi}|M(\theta)|^{2}\left|e^{i \theta}-1\right|^{2} d \theta\right)^{1 / 2}\left(2 \int_{A^{2} / 2}^{\pi}\left|e^{i \theta}-1\right|^{-2}\right)^{1 / 2} \\
\leqslant & \|M\|_{2} A+\left\|M_{0}\right\|_{2} 2 \pi\left(2 \int_{A^{2} / 2}^{\infty} \theta^{-2} d \theta\right)^{1 / 2}=\|M\|_{2} A+2 \pi\left\|M_{0}\right\|_{2} A^{-1} .
\end{aligned}
$$

Letting $A=\left(2 \pi\left\|M_{0}\right\|_{2}\|M\|_{2}^{-1}\right)^{1 / 2}$ we obtain (1.16). Thus, any molecule satisfies $\|M\|_{1} \leqslant 3 \pi$. Incidentally, (1.16) is a version of an inequality of Carlson [9].

It now follows easily that any $f$ satisfying (1.7) does, indeed, belong to Re $H^{1}$. In order to see this it suffices to consider an atom $a(\theta)$ and observe that the associated analytic function $A(z)$ satisfies $\|A\|_{H^{1}} \leqslant \sqrt{2}(1+\pi)$. But this is an immediate consequence of (1.13), (1.14), (1.16) and the well-known fact that $\mu_{1}(F ; r)=\int_{-\pi}^{\pi}\left|F\left(r e^{i \theta}\right)\right| d \theta$ is a nondecreasing function of $r$. Thus, one half of Theorem I is established in case $p=1$.

Similar considerations apply when $p<1$. Note that a $p$-atom is obtained from a 1 -atom $a(\theta)$, when $1 / 2<p \leqslant 1$, by multiplying the latter by $|I|^{1-(1 / p)}$ $\leqslant\|a\|_{2}^{2(1-p) / p}$. Consequently, we define a $p$-molecule (centered at 0$)$, to be a 1 molecule multiplied by its $L^{2}$-norm to the power $2(1-p) / p$. The reader can then check that if $(1.15)$ is replaced by

$$
\|M\|_{2}^{3 p-2}\left\|M\left(1-e^{i \theta}\right)\right\|_{2}^{2-p} \leqslant 1
$$

12 More precisely, $M$ is a 1-molecule centered at the origin; for a molecule centered at $\theta_{0} \in(-\pi, \pi]$ the defining condition is obtained by replacing $\left|e^{i \theta}-1\right|^{2}$ in (1.15) by $\left|e^{i \theta}-e^{i \theta_{0}}\right|^{2}$. 
the above arguments can be easily modified to provide a proof of one of the implications in Theorem I when $2 / 3<p \leqslant 1$.

Let us now return to the study of multiplier operators and the Fourier coefficients of $H^{1}$ functions. We first make the general observation that if $B$ is a linear operator mapping 1-atoms into molecules satisfying (1.15) then, by (1.16), $B$ clearly has a bounded extension as an operator from $\Re e H^{1}$ to $L^{1}(T)$. We can say more, however, by observing that the argument involving the identity (1.14) can be applied to $M=B a(a(\theta)$ being a 1-atom) to show that $M \in \Re e H^{1}$ and its associated analytic function belongs to $H^{1}$ with $H^{1}$ norm not exceeding $2 \pi$ (because of (1.16)). Hence, $B$ maps $\Re$ e $H^{1}$ boundedly into itself. Thus, we see that any linear operator mapping atoms into molecules has an extension that is a bounded operator on $\Re e H^{1}$. In $\$ 3$ we shall show that a molecule belongs to $H^{1}$ in a general setting in which the notion of "analytic $H^{1 "}$ is completely absent.

We are thus led to examine the question: Which operators map atoms into molecules? If the operator is a multiplier two basic properties of the Fourier coefficients $\left\{a_{k}\right\}$ of atoms centered at 0 are particularly useful in trying to resolve this question. The first of these comes from (1.13) and Plancherel's theorem:

$$
\left(\sum_{k=-\infty}^{\infty}\left|a_{k}\right|^{2}\right)^{1 / 2}\left(\sum_{k=-\infty}^{\infty}\left|a_{k+1}-a_{k}\right|^{2}\right) \leqslant \frac{\sqrt{2}}{2 \pi} .
$$

The second one was established in the proof of Hardy's inequality (see the argument following (1.9)) and can be restated as follows:

$$
\left|a_{k}\right| \leqslant \min \left\{1, \frac{k\|a\|_{2}^{-2}}{\pi}\right\}, \quad k=0, \pm 1, \pm 2, \ldots
$$

We shall illustrate the usefulness of these two properties of Fourier coefficients of atoms by establishing the following result:

THEROEM (1.20). Suppose $\left\{\mu_{k}\right\}$ is a bounded sequence satisfying $\left|\mu_{k+1}-\mu_{k}\right|$ $\leqslant c_{0} / k, k=0, \pm 1, \pm 2, \ldots$, then the multiplier operator associated with this sequence maps $\Re$ R $H^{1}$ boundedly into itself. ${ }^{13}$

All we have to show is that if $a(\theta)=\sum_{-\infty}^{\infty} a_{k} e^{i k \theta}$ is an atom then $M(\theta)=\sum_{-\infty}^{\infty} \mu_{k} a_{k} e^{i k \theta}$ satisfies (1.15) independently of $a$. We have:

$$
\|M\|_{2}\left\|M\left(e^{i \theta}-1\right)\right\|_{2}=2 \pi\left(\sum_{-\infty}^{\infty}\left|\mu_{k} a_{k}\right|^{2}\right)^{1 / 2}\left(\sum_{-\infty}^{\infty}\left|\mu_{k+1} a_{k+1}-\mu_{k} a_{k}\right|^{2}\right)^{1 / 2} .
$$

Let $\|\mu\|_{\infty}$ be the $\sup _{k}\left\{\left|\mu_{k}\right|\right\}$ and observe that

$$
\mu_{k+1} a_{k+1}-\mu_{k} a_{k}=\left(\mu_{k+1}-\mu_{k}\right) a_{k+1}+\mu_{k}\left(a_{k+1}-a_{k}\right) .
$$

13 The condition $\left|\mu_{k+1}-\mu_{k}\right| \leqslant c_{0} / k$ implies $2^{N} \sum_{2^{N-1}}^{2^{N}}\left\{\left|\mu_{k+1}-\mu_{k}\right|^{2}+\left|\mu_{-k+1}-\mu_{-k}\right|^{2}\right\} \leqslant c_{0}^{\prime}$ for all $N>0$. Such a condition is of ten called a Hörmander condition (see [36]). The proof we give can easily be changed to show that the Hörmander condition yields the conclusion of $(1.20)$. Similar theorems are also announced in $[29$, p. 150]. 
Thus the product involving the two series above is dominated by

$$
\begin{aligned}
& \left(\sum_{-\infty}^{\infty}\left|\mu_{k} a_{k}\right|^{2}\right)^{1 / 2}\left(\sum_{-\infty}^{\infty}\left|\mu_{k}\right|^{2}\left|a_{k+1}-a_{k}\right|^{2}\right)^{1 / 2} \\
& +\left(\sum_{-\infty}^{\infty}\left|\mu_{k} a_{k}\right|^{2}\right)^{1 / 2}\left(\sum_{-\infty}^{\infty}\left|\mu_{k+1}-\mu_{k}\right|^{2}\left|a_{k+1}\right|^{2}\right)^{1 / 2} .
\end{aligned}
$$

By (1.18) we see that the first summand does not exceed $\sqrt{2}\|\mu\|_{\infty}^{2} / 2 \pi$. Using (1.19), on the other hand, we have

$$
\begin{aligned}
\left(\sum_{-\infty}^{\infty}\left|\mu_{k} a_{k}\right|^{2}\right) & \left(\sum_{-\infty}^{\infty}\left|\mu_{k+1}-\mu_{k}\right|^{2}\left|a_{k+1}\right|^{2}\right) \\
& \leqslant\|\mu\|_{\infty}^{2}\left(\sum_{-\infty}^{\infty}\left|a_{k}\right|^{2}\right)\left(\frac{c_{0}^{2}}{\pi^{2}} \sum_{|k| \leqslant\|a\|_{2}} \frac{1}{k^{2}} k^{2}\|a\|_{2}^{-4}+\sum_{|k| \geqslant\|a\|_{2}^{2}} \frac{c_{0}^{2}}{k^{2}}\right) .
\end{aligned}
$$

But this last expression is clearly less than a constant depending only on $\|\mu\|_{\infty}$ and $c_{0}$ and Theorem (1.20) is proved.

When $p<1, \operatorname{Re} H^{p}$ is not naturally converted into a Banach space. Nevertheless, we can introduce a "norm" by letting $\|f\|_{H^{p}} \equiv\|F\|_{H^{p}}$, where $F$ is the analytic function associated with the distribution $f$ and $\operatorname{Im} F(0)=0$ (see (1.13)). This is not a norm; however, $d_{p}(f, g)=\|f-g\|_{p}^{p}$ defines a metric. Thus, we can discuss continuity questions concerning operators on $\operatorname{Re} H^{p}$. In particular, a linear operator mapping $p$-atoms into $p$-molecules is continuous on $\operatorname{Re} H^{p}, 1 / 2<p \leqslant 1$. We can translate (1.17) into a condition on the Fourier coefficients of a $p$-atom that is analogous to (1.18). Condition (1.19) also has a simple analog. All this can be stated by saying that the Fourier coefficients $\left\{a_{k}\right\}$ of a $p$-atom $a(\theta), 1 / 2<p \leqslant 1$, are such that $\left\{\|a\|_{2}^{p /(2-p)-1} a_{k}\right\}$ are the Fourier coefficients of a 1-atom. Using these facts one can show that the assumptions in Theorem (1.20) imply that the multiplier operator is bounded on $\operatorname{Re} H^{p}$ as long as $2 / 3<p \leqslant 1$.

Several examples of multipliers satisfying the hypotheses of Theorem (1.20) can be given. The case where $\mu_{k}=|k|^{i \gamma}, \gamma$ real, has been studied by several people. The operator associating to $f \in \operatorname{Re} H^{1}$ the boundary value $F$ of the analytic function in $H^{1}$ such that $R e F=f$ is a multiplier operator given by $\mu_{k}=0$ if $k<0, \mu_{0}=1$ and $\mu_{k}=2$ if $k>0$. The fact that $H^{1}$ is stable under the action of certain convolution operators was first pointed out by $\mathrm{E}$. M. Stein. (See [54] and [55]; there this situation is discussed in higher dimensional settings. We shall do the same later on.)

Several considerations are in order once the continuity of such operators on the spaces $\operatorname{Re} H^{p}, p \leqslant 1$, is established. First, we remark that a duality argument shows that the corresponding Lipschitz spaces (see (1.12)) are preserved.

Secondly, the question of interpolating the boundedness of these operators becomes a natural one. We have already remarked that any such multiplier operator is bounded on $L^{2}(T)$. At this point we would like to point out that here, again, the atomic characterization of $\Re e H^{p}$ is particularly well suited for obtaining a variety of results on interpolation of operators. We shall develop 
this topic later in a more general setting. For the moment, however, we shall illustrate the simplicity of some of the methods involved by examining some aspects of the following theorem:

THeorem (1.21). Fix $p_{0} \leqslant 1$. Let $B$ be a bounded linear operator on $L^{2}(T)$ satisfying the condition

$$
\|B a\|_{p_{0}} \leqslant c
$$

for all $p_{0^{-}}$atoms. Then, $\|B f\|_{p} \leqslant c_{p}\|f\|_{H^{p}}$ for $p_{0} \leqslant p \leqslant 1$, and $\|B f\|_{p} \leqslant a_{p}\|f\|_{p}$ for $1<p \leqslant 2$, where $c_{p}$ and $a_{p}$ are independent of $f \in H^{p}$ or $f \in L^{p}(T)$ (though they do depend on $c$ in (1.22) and $p_{0}$ ).

To illustrate our point we shall only consider the case $1 / 2<p_{0}<1$ and we shall deduce the conclusion for $p=1$. As we have already seen on several occasions it suffices to obtain the result for $f(\theta)=a(\theta)$ a 1-atom. Then $|I|^{1-\left(1 / p_{0}\right)} a$ is a $p_{0}$-atom ( $I$ being the supporting interval of $\left.a\right)$. Thus, by (1.22) and the $L^{2}$-boundedness of $B$ we obtain

$$
\|B a\|_{p_{0}} \leqslant c|I|^{\left(1 / p_{0}\right)-1} \text { and }\|B a\|_{2} \leqslant c^{\prime}|I|^{-1 / 2} .
$$

Using Hölder's inequality and (1.23) we obtain the desired conclusion:

$$
\begin{aligned}
\int|B a| & =\int|B a|^{p_{0} /\left(2-p_{0}\right)}|B a|^{\left(2-2 p_{0}\right) /\left(2-p_{0}\right)} \\
& \leqslant\left(\int|B a|^{p_{0}}\right)^{1 /\left(2-p_{0}\right)}\left(\int|B a|^{2}\right)^{\left(1-p_{0}\right) /\left(2-p_{0}\right)} \\
& \leqslant c c^{\prime}|I|^{\left(1-p_{0}\right) /\left(2-p_{0}\right)}|I|^{\left(p_{0}-1\right) /\left(2-p_{0}\right)}=c c^{\prime} .
\end{aligned}
$$

We thus see that the boundedness of many operators on $H^{p}$, or merely on atoms, for some $p<1$, implies its continuity as an operator on $L^{q}$ for certain $q>1$. In particular, the familiar results of Calderon-Zygmund (see [5]) have a natural setting in this atomic approach to $H^{p}$ spaces. In fact, let $k(\theta)$ be integrable for $0<\varepsilon \leqslant|\theta| \leqslant \pi$ for all $\varepsilon>0$ and satisfy the two conditions

(a) the sequence $\hat{k}(j)=\lim _{\varepsilon \rightarrow 0} \int_{\varepsilon<|\theta| \leqslant \pi} e^{-i j \theta} \cdot k(\theta) d \theta$ exists and is bounded by $c_{1}$;

(b) $\int_{2|\theta|<|\psi| \leqslant \pi}|k(\psi-\theta)-k(\psi)| d \psi \leqslant c_{2}$.

Let us also assume that the convolution $\int_{-\pi}^{\pi} k(\theta-\varphi) f(\varphi) d \varphi$ is suitably defined for $f \in L^{2}(T)$. We shall show that

$$
\|k * a\|_{1}=\int_{-\pi}^{\pi}\left|\int_{-\pi}^{\pi} k(\theta-\varphi) a(\varphi) d \varphi\right| d \theta \leqslant c_{1}+c_{2}
$$

for all 1-atoms $a$. From this it will follow that there exists a constant $c_{p}$ such that

$$
\|k * a\|_{p} \leqslant c_{p}\|f\|_{p}
$$

whenever $1<p<\infty$.

Before doing this we would like to point out that these considerations 
provide us yet another method for showing that the atomic $\operatorname{Re} H^{1}$ space is, indeed, included in the real part of the classical $H^{1}$ space. This follows from the fact that the kernel $k(\theta)=\left(1+e^{i \theta}\right) /\left(1-e^{i \theta}\right) \sim 1+2 \sum_{j=1}^{\infty} e^{i j \theta}$ obviously maps (as a convolution operator) $f$ into the boundary value of the analytic function having real part $f$ (for any reasonably "nice" $f$ ) and, moreover, the mean value theorem yields the inequality

$$
|k(\psi-\theta)-k(\psi)| \leqslant c|\theta| /|\psi|^{2}, \text { if }|\psi|>2|\theta|,
$$

which certainly implies (b).

To establish (1.24) we argue as follows: First observe that condition (a) tells us that $f \rightarrow k * f$ is a bounded operator on $L^{2}(T)$. Now let $a(\theta)$ be a 1-atom supported in an interval $I$ centered at 0 . Since the result is trivial for the constant atom, we can assume $\int_{-\pi}^{\pi} a=0$. Then,

$$
\begin{aligned}
\|k * a\|_{1} & =\int_{-\pi}^{\pi}|(k * a)(\psi)| d \psi \\
& =\int_{2|I|<|\psi| \leqslant \pi}|(k * a)(\psi)| d \psi+\int_{2|I| \geqslant|\psi|}|(k * a)(\psi)| d \psi .
\end{aligned}
$$

To estimate the first of these integrals we make use of the fact that $a$ has mean 0 and, thus, the integral equals

$$
\begin{aligned}
& \int_{2|I|<|\psi| \leqslant \pi}\left|\int_{-\pi}^{\pi}[k(\psi-\theta)-k(\psi)] a(\theta) d \theta\right| d \psi \\
& \leqslant \int_{|I|}|a(\theta)|\left(\int_{2|I|<|\psi| \leqslant \pi}|k(\psi-\theta)-k(\psi)| d \psi\right) d \theta .
\end{aligned}
$$

But, by condition (b), $\int_{2|I|<|\psi| \leqslant \pi}|k(\psi-\theta)-k(\psi)| d \psi \leqslant c_{2}$ and we obtain the inequality

$$
\int_{2|I|<|\psi| \leqslant \pi}|(k * a)(\psi)| d \psi \leqslant c_{2} \int_{I}|a(\theta)| d \theta \leqslant c_{2} .
$$

To estimate the second integral we use Schwarz's inequality and the $L^{2}$. boundedness of the convolution operator

$$
\int_{|\psi| \leqslant 2|I|}|k * a| \leqslant\|k * a\|_{2} 2|I|^{1 / 2} \leqslant c_{1}\|a\|_{2}|I|^{1 / 2} \leqslant c_{1}
$$

and (1.24) is established. Inequality (1.25) then follows by interpolation and duality (since convolution operators are the negative of their adjoints).

Let us summarize very briefly some of the points we have made favoring the study of $H^{p}$ spaces, $p \leqslant 1$, and, in particular, the atomic approach to this study:

(1) Many results that are valid for $L^{q}(T), 1<q<\infty$, fail for $L^{1}(T)$ but remain valid for $\Re e H^{1}$ (or $\Re e H^{p}$ ).

(2) Several estimates on operators (in particular, convolution operators) are easily made on atoms and imply corresponding estimates for these operators applied to general functions. 
(3) We can characterize the Fourier coefficients of the distributions $f$ $\in \Re$ e $H^{p}$ and from this obtain results on multiplier operators. ${ }^{14}$

(4) The boundedness of some operator on $\Re e H^{p}, p \leqslant 1$, implies (via interpolation theorems) the boundedness of this operator on certain $L^{q}(T)$ spaces, $1<q$. Moreover, by duality we see that such an operator must preserve certain smoothness properties. Atoms are very useful for obtaining such results easily.

Let us now describe briefly the Hardy spaces associated with the real line $\mathbf{R}$ and their atomic characterization. The classical definition of these spaces is the following one: $F \in H^{p}(\mathbf{R}), 0<p$, if and only if $F$ is an analytic function defined on the upper half-plane $\mathbf{R}_{+}^{2}=\left\{z=x+i y \in \mathbf{R}^{2} ; x \in \mathbf{R}, y>0\right\}$ satisfying

$$
\sup _{y>0}\left(\int_{-\infty}^{\infty}|F(x+i y)|^{p} d x\right)^{1 / p} \equiv\|F\|_{H^{p}}<\infty .
$$

Their theory was developed by Krylov [44]. In particular, it is known that the boundary values

$$
\lim _{y \rightarrow 0+} F(x+i y)=F(x)
$$

exist almost everywhere and in the $L^{p}$ norm $(p>0)$. As was the case in the circle, these boundary values should be considered as distributions when $p<1$ and, in this case, their real parts can be characterized in terms of atoms that can be defined in a manner that is completely analogous to the one we gave above. This characterization applies to the case $p=1$ as well. We shall now, however, introduce a variant of the notion of an atom that has the advantage of having a completely characterizable Fourier transform:

Definition. A function $a \in L^{2}(\mathbf{R})$ is a $(p, 2)$-atom, $p \leqslant 1$, if

(i) it is supported in a finite interval $I$;

(ii) $\left(\int_{I}|a(x)|^{2} d x /|I|\right)^{1 / 2} \leqslant|I|^{-1 / p}$;

(iii) $\int_{-\infty}^{\infty} x^{k} a(x) d x=0$ for $k=0,1, \ldots,[1 / p]-1$, where $[\alpha]$ denotes the largest integer less than or equal to $\alpha .^{15}$

With this definition the statement of Theorem I on $\mathbf{R}$ remains unchanged and the $H^{p}(\mathbf{R})$ analog of (1.8) holds.

Recall that we had made the assumption that $L^{p}(T)$ consisted of real-valued functions and $\Re e H^{p}$ consisted of the boundary values of the real parts of $H^{p}$ functions on the unit disc. We shall abandon this convention and we allow our atoms to be complex-valued. $H^{p}(\mathbf{R})$ will denote the complexification of the

14 We are aware of the fact that despite announcing a characterization of the Fourier coefficients of functions $f \in \Re$ Re $H^{1}$ we have not yet given a characterization. Actually, for our purposes, we needed only to know properties (1.18) and (1.19) for Fourier coefficients of atoms. The reader can easily verify that $\sum c_{k} e^{i k \theta}$ is a Fourier series of $f \in \operatorname{Re} H^{\mathbf{1}}$ if and only if $c_{k}=\sum \lambda_{n} e^{i \alpha_{n} k} a_{k}^{(n)}$ where $\sum\left|\lambda_{n}\right|<\infty$; each sequence $a^{(n)}=\left\{\ldots, a_{-2}^{(n)}, a_{-1}^{(n)}, a_{0}^{(n)}, a_{1}^{(n)}, a_{2}^{(n)}, \ldots\right\}$ satisfies (1.18) and (1.19); the numbers $\alpha_{n}$ reflect the fact that we translated the atoms in the decomposition (1.7) so that their supporting interval is centered at 0 .

15 That is, as $p$ tends to zero we impose the condition that more moments of $a$ are 0 . Similar conditions on the $p$-atoms on $T$ are the ones that are needed for Theorem I when $p \leqslant 1 / 2$ (see [10]). 
space $R H^{p}(\mathbf{R})$. This space (of distributions) should not be confused with the space of boundary values of analytic functions satisfying (1.26).

Let us now suppose $a(t)$ is a $(p, 2)$-atom supported on an interval $I$ which is centered about the origin. There is no loss in generality if we assume $\|a\|_{2}^{2}=|I|^{(p-2) / p}$. Then the function $A(z)=(1 / 2 \pi) \int_{-\infty}^{\infty} e^{-i z t} a(t) d t$ is an entire function in the complex plane C. Moreover, $|A(z)| \leqslant e^{|z||I|}\|a\|_{1}$; that is, $A$ is of exponential type. The Plancherel theorem and (ii) yield $\int_{-\infty}^{\infty}|A(x)|^{2} d x$ $\leqslant|I|^{1-(2 / p)}$; from (iii) we also have the fact that the derivatives $A^{(k)}(0)$ $=0$ for $k=0,1, \ldots,[1 / p]-1$. It follows from the Paley-Wiener theorem (see (7.2) in Vol. II of [64]) that the converse of all this is also true: If $A \in L^{2}(\mathbf{R})$ is the restriction of an entire function of exponential type $\sigma$, is normalized by $\|A\|_{2}^{2}=\sigma^{1-(2 / p)}$ and satisfies $A^{(k)}(0)=0$ for $k=0,1, \ldots$, $[1 / p]-1$, then $A$ is the Fourier transform of a $(p, 2)$-atom. This gives us the following characterization of the Fourier transforms of the elements of $H^{p}(\mathbf{R})$ :

THEOREM II. $\hat{f}$ is the Fourier transform of a member of $H^{p}(\mathbf{R}), p \leqslant 1$, if and only if

$$
\hat{f}=\sum_{j=0}^{\infty} \lambda_{j} e^{i \alpha_{j} x} A_{j}(x)
$$

where $\sum\left|\lambda_{j}\right|^{p}<\infty, \alpha_{j}$ are real numbers and $A_{j}(x)$ are the restrictions of entire functions of exponential type $\sigma_{j}$ such that $\left\|A_{j}\right\|_{2}^{2}=\sigma_{j}^{1-(2 / p)} ;$ moreover, $A_{j}^{(k)}(0)$ $=0$ for $0 \leqslant k \leqslant[1 / p]-1$.

In complete analogy with $(1.13)$ any $(1,2)$-atom $a(t)$ satisfies $\|a\|_{2}\|t a(t)\|_{2}$ $\leqslant 1$. Since the Fourier transform of $t a(t)$ is $-i A^{\prime}(x)$ it follows from Plancherel's theorem that $\|A\|_{2}\left\|A^{\prime}\right\|_{2} \leqslant 1$. We are led, as before, to introduce the notions of a molecule and of a Fourier-molecule centered at 0 . We define the latter to be a function $\mathfrak{M}$ on $\mathbf{R}$ satisfying
(i) $\|\mathfrak{M}\|_{2}\left\|\mathfrak{M}^{\prime}\right\|_{2} \leqslant 1$,
(ii) $|\mathfrak{M}(x)| \leqslant|x| /\|\mathfrak{M}\|_{2}^{2}$.

We leave it to the reader to verify that $A=\hat{a}$ satisfies (ii) when $a$ is a $(2,1)$ atom centered at 0 , that $\breve{\mathfrak{R}}+i(\operatorname{sgn} \mathfrak{M})^{\swarrow} \in L^{1}$ with uniformly bounded $L^{1}$ norm (thus, $\breve{\mathfrak{M}}$ is in $H^{1}(\mathbf{R})$ ) and, finally, that Theore $\mathrm{m}$ II holds if we replace the functions $A_{j}$ by Fourier-molecules.

For $2 / 3<p \leqslant 1$ we say that $\mathfrak{M}$ is a Fourier-molecule for $H^{p}(\mathbf{R})$ (centered at 0$)$ if $\sigma^{1-(1 / p)} \mathfrak{M}$ is a Fourier-molecule for $H^{1}(\mathbf{R})$, where $\|\mathfrak{M}\|_{2}^{2}=\sigma^{(2 / p)-1}$. Again, it can be easily verified that Theorem II can be restated in terms of Fourier-molecules (similar definitions can be given for $p<2 / 3$ ).

Theorem (1.20) has the following analog:

THEOREM (1.29). The multiplier operator mapping $f \in H^{p}(\mathbf{R})$ into $(\mu \hat{f})^{\searrow}$ is bounded on $H^{p}(\mathbf{R})$ when $2 / 3<p$, provided $\mu \in L^{\infty}(\mathbf{R})$ satisfies $\sup _{R>0} R \int_{R \leqslant|x|<2 R}\left|\mu^{\prime}(x)\right|^{2} d x<\infty$.

This result follows immediately from the observation that the conditions we just imposed on $\mu$ are necessary and sufficient for the multiplier to preserve the defining properties (1.28) of the Fourier-molecules in question (the slight alteration of the inequalities (i) and (ii) by fixed constants depending only on $\mu$ are of minor importance). 
Another application of Theorem II stated in terms of Fourier-molecules follows from the simple general inequality $\left.|| \mathfrak{M}\right|^{\prime}|\leqslant| \mathfrak{M}^{\prime} \mid$ valid for any differentiable function $\mathfrak{M}$. Since this inequality implies that $|\mathfrak{M}|$ is a Fouriermolecule whenever $\mathfrak{M}$ is one we have

THEOREM (1.30). Suppose $f \in H^{1}(\mathbf{R})$. Then there exists $g \in H^{1}(\mathbf{R})$ such that $|\hat{f}| \leqslant \hat{g}$ and $\|g\|_{H^{1}} \leqslant c\|f\|_{H^{1}}$, where $c$ is independent of $f$.

(See p. 287 of Vol. I of [64] for the version of this result on T.) We shall restrict ourselves to these remarks concerning $H^{p}(\mathbf{R})$. It will become clear in the sequel that the atomic approach plays an important role here as well: duality, the study of other operators commuting with translations, maximal operators and other subjects can be studied from this point of view.

We shall now extend these notions so that we can see how they apply to the study of convolution operators on $n$-dimensional Euclidean space $\mathbf{R}^{n}$. Let us begin by describing an extension of the theory of Hardy spaces to $\mathbf{R}^{n}$ developed in [59]. The first observation that motivated this extension is that the Cauchy-Riemann equations admit the following natural generalization to higher dimensions: A vector-valued function $F(x, y)=\left(u_{0}(x, y), u_{1}(x, y), \ldots\right.$, $\left.u_{n}(x, y)\right)$ defined on the upper half-space $\mathbf{R}_{+}^{n+1}=\left\{(x, y) \in \mathbf{R}^{n+1}: x \in \mathbf{R}^{n}, y\right.$ $>0\}$ is called a generalized analytic function or a Riesz system if and only if $u_{j}, j=0,1, \ldots, n$, are harmonic functions satisfying:

$$
\sum_{j=0}^{n} \frac{\partial u_{j}}{\partial x_{j}}=0 \text { and } \frac{\partial u_{j}}{\partial x_{k}}=\frac{\partial u_{k}}{\partial x_{j}}
$$

where $\partial / \partial x_{0}$ is defined to be $\partial / \partial y$.

Such a generalized analytic function $F$ is said to belong to $H^{p}\left(\mathbf{R}^{n}\right), p>0$, provided

$$
\begin{aligned}
\|F\|_{H^{p}} & \equiv \sup _{y>0}\left(\int_{\mathbf{R}^{n}}|F(x, y)|^{p} d x\right)^{1 / p} \\
& \equiv \sup _{y>0}\left(\int_{\mathbf{R}^{n}}\left[\sum_{j=0}^{n}\left|u_{j}(x, y)\right|^{2}\right]^{p / 2} d x\right)^{1 / p}<\infty .
\end{aligned}
$$

It can be shown that if $p>(n-1) / n$ then $\lim _{y \rightarrow 0} F(x, y)=\left(f_{0}(x), f_{1}(x), \ldots\right.$, $\left.f_{n}(x)\right)=F(x)$ exists a.e. and in the $L^{p}$-norm. As is the case in one dimension,when $p<1$ one should consider these boundary values in the sense of distributions. When $p=1$, however, $F(x)$ is a bona fide vector-valued function in $L^{1}\left(\mathbf{R}^{n}\right)$ and its components are related by the Riesz transforms. In terms of the Fourier transform these relations can be expressed in the following way:

$$
\hat{f}_{j}(x)=c_{n}\left(x_{j} /|x|\right) \hat{f}_{0}(x), \quad j=1,2, \ldots, n .
$$

Moreover,

$$
\left(P^{*} F\right)(x)=\sup _{|t-x|<y}|F(t, y)|
$$

belongs to $L^{p}\left(\mathbf{R}^{n}\right)$ whenever $F \in H^{p}\left(\mathbf{R}^{n}\right),(n-1) / n<p$ (see [59] for all these results). 
C. Fefferman and E. M. Stein [29] recently proved that this property actually characterizes the distributions that are boundary values of functions in $H^{p}\left(\mathbf{R}^{n}\right)$. More precisely, a distribution $f$ is the boundary value of the first component of a Riesz system $F \in H^{p}$ if and only if

$$
\left(P^{*} f\right)(x)=\sup _{|t-x|<y}\left|\left(P_{y}^{*} f\right)(t)\right|
$$

belongs to $L^{p}\left(\mathbf{R}^{n}\right)$, where $P_{y}^{*} f$ is the Poisson integral of $f$ :

$$
c_{n} \int_{\mathbf{R}^{n}} f(s) \frac{y}{\left[|s-t|^{2}+y^{2}\right]^{(n+1) / 2}} d s .
$$

We shall call such an $f$ a boundary distribution of $H^{p}\left(\mathbf{R}^{n}\right)$. Other maximal operators, besides the maximal Poisson integral (1.33) can be used for this characterization. For example, if $\varphi \geqslant 0$ is a $C^{\infty}$ function with compact support and $\varphi_{\varepsilon}(x)=\varepsilon^{-n} \varphi\left(\varepsilon^{-1} x\right)$ then $\sup _{\varepsilon}>0\left|\left(f * \varphi_{\varepsilon}\right)(x)\right|$ belongs to $L^{p}$ if and only if $f$ is a boundary distribution of $H^{p}\left(\mathbf{R}^{n}\right)$. The discovery of this "maximal function" characterization of $H^{p}\left(\mathbf{R}^{n}\right)$ was an important breakthrough in the real-variable understanding of these spaces as well as in the study of operators on them.

An interesting application of the Fefferman-Stein results is the observation made by $\mathrm{L}$. Carleson [8] that $H^{p}\left(\mathbf{R}^{n}\right)$ can also be defined in terms of holomorphic functions of several complex variables. In fact, let $\Gamma_{j}, j=1,2$, $\ldots, n+1$, be open convex cones with vertex at the origin such that their union covers $\mathbf{R}^{n}$, except for the origin. Assume that none of the $\Gamma_{j}$ contains an entire straight line. Then $f$ is a boundary distribution for $H^{p}\left(\mathbf{R}^{n}\right)$ if and only if

$$
f=f_{1}+f_{2}+\cdots+f_{n+1}
$$

where each $f_{j}$ is a boundary distribution of a holomorphic function $F_{j}$ in the $H^{p}$ space associated with the tube domain $T_{\Gamma_{i}^{*}} .{ }^{16}$

Briefly, we obtain this representation in the following way: Suppose $f$ is a boundary distribution of $H^{p}\left(\mathbf{R}^{n}\right)$. We can construct $n+1$ functions $\varphi_{j}$ that are homogeneous of degree 0 such that $\sum_{j=1}^{n} \varphi_{j}(x) \equiv 1(x \neq 0)$, the support of $\varphi_{j}$ lies in $\Gamma_{j}^{*}$ and each $\varphi_{j}$ belongs to the class $C^{\infty}\left(\mathbf{R}^{n}-\{0\}\right)$. It follows from the Fefferman-Stein theory that $f_{j}=\left(\varphi_{j} \hat{f}\right)^{\sim}$ is also a boundary distribution of $H^{p}\left(\mathbf{R}^{n}\right)$. The integral representation of $F_{j}$ in terms of $\hat{f}_{j}$ (see footnote 16) is then in $H^{p}\left(T_{\Gamma_{j}^{*}}\right)$. Conversely, if $f_{j}$ is the boundary value of a function in $H^{p}\left(T_{\Gamma_{j}^{*}}\right)$ it follows from the theory of Hardy spaces on tubes that a Poisson-type maximal function of $f_{j}$ belongs to $L^{p}\left(\mathbf{R}^{n}\right)$. This, in turn, forces $f_{j}$ to be a boundary distribution of $H^{p}\left(\mathbf{R}^{n}\right)$ because of the Fefferman-Stein theory.

There are several other extensions of Hardy spaces in terms of generalized Cauchy-Riemann equations (see [6] and [60]). ${ }^{17}$ Fefferman and Stein have

16 These are yet another extension of Hardy spaces to the $n$-dimensional case. We shall not consider them further in this paper. For their definition and properties see Chapter III of [57]. In this case $\Gamma_{j}^{*}$ is the cone dual to $\Gamma_{j}$ and $T_{\Gamma_{j}^{*}}=\left\{z=x+i y \in \mathbf{C}^{n}: x \in \mathbf{R}^{n}\right.$ and $\left.y \in \Gamma_{j}^{*}\right\} . f_{j}$ and $F_{j}$ are related as follows: the support of $\hat{f}$ lies in $\Gamma_{j}$,

$$
F_{j}(z)=\int_{\Gamma_{j}} e^{i z \cdot t} \hat{f}_{j}(t) d t \text { and } \sup _{y \in \Gamma_{j}^{*}} \int_{\mathbf{R}^{n}}|F(x+i y)|^{p} d x<\infty .
$$


shown that their maximal function characterization remains valid for these extensions. Moreover, they show that the dual of $H^{1}\left(\mathbf{R}^{n}\right)$ is the space $B M O\left(\mathbf{R}^{n}\right)$ of functions of bounded mean oscillation on $\mathbf{R}^{n}$ (the definition of this space is the same as in the one dimensional case except that intervals are replaced by solid spheres). Atoms on $\mathbf{R}^{n}$ can also be introduced as before (using solid spheres instead of intervals). Thus, the atomic $\mathfrak{S}_{\mathcal{G}}^{1}\left(\mathbf{R}^{n}\right)$ space is well defined on $\mathbf{R}^{n}$ : it consists of all functions $f=\sum_{j=1}^{\infty} \lambda_{j} a_{j}$, where $\sum\left|\lambda_{j}\right|<\infty$ and each $a_{j}$ is an atom (either a 1-atom, defined similarly to those introduced when discussing $T$, or a $(1,2)$-atom. We shall show later that this makes no difference). If we define the norm of $f,\|f\|_{\mathfrak{S}^{1}\left(\mathbf{R}^{n}\right)} \equiv\|f\|_{\mathfrak{S}_{1}}$, to be the infimum of all the sums $\Sigma\left|\lambda_{j}\right|$ for which $f=\sum \lambda_{j} a_{j}$ we obtain a Banach space. Similarly the atomic $\mathfrak{S}^{p}\left(\mathbf{R}^{n}\right)$ space can be introduced by making use of $p$ atoms. We shall do all this carefully in a much more general setting. We end this section by making some observations concerning these spaces. The first one is that the elements of $\mathfrak{S}^{p}\left(\mathbf{R}^{n}\right)$ are always boundary distributions of $H^{p}\left(\mathbf{R}^{n}\right)$ when $n /(n+1)<p \leqslant 1$. This follows easily from the general theory of $H^{p}\left(\mathbf{R}^{n}\right)$ spaces developed in [59] once the following inequality is established:

$$
\text { If } a \text { is a p-atom, } n /(n+1)<p \leqslant 1 \text {, then }\left\|R_{j} a\right\|_{p} \leqslant c_{p, n},
$$

where $R_{j}$ is the $j$ th Riesz transform of $a$ and $c_{p, n}$ is a constant independent of the atom $a$. This is as easy to show as was (1.24): $R_{j}$ is the integral operator with kernel $k_{j}(x)=x_{j} /|x|^{n+1}, j=1,2, \ldots, n$ (up to a multiplicative constant). $\left(R_{j} a\right)(x)$ is, then, the principal value integral

$$
\lim _{\varepsilon \rightarrow 0+} \int_{|x-y| \geqslant \varepsilon} k_{j}(x-y) a(y) d y \equiv \text { P.V. } \int_{\mathbf{R}^{n}} k_{j}(x-y) a(y) d y .
$$

An immediate calculation using the mean value theorem yields

$$
\left|k_{j}(x-y)-k_{j}(x)\right| \leqslant(n+2) 2^{n} \frac{|y|}{|x|^{n+1}} \text { if } 2|y|<|x| .
$$

Because of the translation invariance of the convolution operator $R_{j}$ there is no loss in generality if we assume $a$ is supported in the sphere $S_{r}$ about the origin of radius $r>0$ and $|a(x)| \leqslant\left|S_{r}\right|^{-\mathrm{I} / p}=c r^{-n / p}$ (from here on let us agree that $c$ denotes a constant, not always the same one, that is independent of the atom $a$ ). We have

$$
\begin{aligned}
\left\|R_{j} a\right\|_{p}^{p} & =\int_{\mathbf{R}^{n}} \mid \text { P.V. }\left.\int_{|y|<r} k_{j}(x-y) a(y) d y\right|^{p} d x \\
& =\left(\int_{|x| \leqslant 2 r}+\int_{|x|>2 r}\right) \mid \text { P. V. }\left.\int_{|y|<r} k_{j}(x-y) a(y) d y\right|^{p} d x \equiv \text { I + II . }
\end{aligned}
$$

We estimate I by using Hölder's inequality and the $L^{2}$-boundedness of $R_{j}$ :

$$
\begin{aligned}
\mathrm{I} & =\int_{|x| \leqslant 2 r} 1 \cdot\left|\left(R_{j} a\right)(x)\right|^{p} d x \leqslant c\left(r^{n}\right)^{(2-p) / 2}\left(\int_{\mathbf{R}^{n}}\left|\left(R_{j} a\right)(x)\right|^{2} d x\right)^{p / 2} \\
& \leqslant c r^{n(2-p) / 2}\|a\|_{2}^{p} \leqslant c r^{n(2-p) / 2} r^{n(p-2) / 2}=c .
\end{aligned}
$$

17 In fact, this approach can be carried out even in the setting of compact Lie groups [16]. 
We estimate II by making use of the fact that $a$ has mean 0 and property (1.36):

$$
\begin{aligned}
\mathrm{II} & =\int_{|x|>2 r}\left|\int_{|y|<r}[k(x-y)-k(x)] a(y) d y\right|^{p} d x \\
& \leqslant c_{n}^{p} \int_{|x|>2 r}|x|^{-p(n+1)}\left\{\int_{|y|<r}|y||a(y)| d y\right\}^{p} d x \\
& \leqslant c \int_{2 r}^{\infty} s^{n-1-p(n+1)} d s\left(\int_{|y|<r}|y| r^{-n / p} d y\right)^{p} \leqslant c r^{n-p(n+1)} r^{-n+p(n+1)}=c .
\end{aligned}
$$

This establishes (1.35).

Let us examine some of the consequences of (1.35) when $p=1$. By a simple extension of the reasoning used to establish (1.11) it follows that $B M O\left(\mathbf{R}^{n}\right)$ represents the dual of $\mathfrak{S}^{1}\left(\mathbf{R}^{n}\right)$. In particular, $\|f\|_{\mathfrak{Q}^{1}\left(\mathbf{R}^{n}\right)}$ is equivalent to the norm obtained by considering the maximum of all values $L f$ where $L$ is a bounded linear functional on $\mathfrak{S}^{1}\left(\mathbf{R}^{n}\right)$ represented by a BMO function having BMO norm 1. But, as we stated above, Fefferman and Stein showed that this last norm is equivalent to that of $H^{1}\left(\mathbf{R}^{n}\right)$. Thus, (1.35) shows that $\mathfrak{S}^{1}\left(\mathbf{R}^{n}\right)$ is not only contained in $H^{1}\left(\mathbf{R}^{n}\right)$ but the two norms are equivalent. Moreover, it follows from the work of Fefferman and Stein that $B M O$ represents $\left(H^{1}\left(\mathbf{R}^{n}\right)\right)^{*}$ in the same manner it represents $\left(\mathscr{S}_{\mathcal{E}}^{1}\left(\mathbf{R}^{n}\right)\right)^{*}$ (see $\left.\S 3\right)$. The Hahn-Banach theorem can be invoked to argue that $\mathfrak{S}^{1}\left(\mathbf{R}^{n}\right)=H^{1}\left(\mathbf{R}^{n}\right)$.

For $p<1$ the identification of $\mathfrak{S}^{p}$ with $H^{p}$ cannot be done using duality. The decomposition theorem obtained in [10] has recently been extended by $\mathrm{R}$. Latter to $\mathbf{R}^{n}$ and provides a direct proof that any $f \in H^{p}$ admits an atomic decomposition; that is, $\mathfrak{S}^{p}=H^{p}{ }^{18}$

2. Hardy spaces associated with spaces of homogeneous type. In [17] we introduced certain topological measure spaces having properties that permitted us to extend the Calderón-Zygmund theory of singular integrals to these spaces. It turns out that these properties can also be used to obtain a very useful theory if we employ Theorem I in order to define Hardy spaces. As can be seen from $\$ 1$, by doing this we completely avoid the problem of finding an appropriate system of partial differential equations generalizing the CauchyRiemann equations. Nevertheless, we shall see that in certain examples, where the structure of the space allows us to introduce natural extensions of the Cauchy-Riemann equations, the spaces introduced here are closely related to Hardy spaces that could be defined in terms of such partial differential equations.

We begin by introducing the notion of a space of homogeneous type: this is a topological space $X$ endowed with a Borel measure $\mu$ and a quasi-metric (or quasi-distance) $d$. The latter is a mapping $d: X \times X \rightarrow \mathbf{R}^{+}=\{t \in \mathbf{R}: t \geqslant 0\}$ satisfying (a) $d(x, y)=d(y, x)$, (b) $d(x, y)>0$ if and only if $x \neq y$ and (c) there exists a constant $K$ such that $d(x, y) \leqslant K[d(x, z)+d(z, y)]$ for all $x, y, z$ in $X$. We postulate that the spheres $B_{r}(x)=\{y \in X: d(x, y)<r\}$ centered at

${ }^{18}$ Latter's proof was communicated to us by a letter from J. Garnett while this manuscript was still being written. 
$x$ and of radius $r>0$ form a basis of open neighborhoods of the point $x$ and, also, $\mu\left(B_{r}(x)\right)>0$ whenever $r>0$. Our basic assumption relating the measure and the quasi-distance is the existence of a constant $A$ such that

$$
\mu\left(B_{r}(x)\right) \leqslant A \mu\left(B_{r / 2}(x)\right)
$$

Before developing the theory of Hardy spaces in this setting we shall give several examples of spaces of homogeneous type.

(1) $X=\mathbf{R}^{n}, d(x, y)=|x-y|=\left(\sum_{j=1}^{n}\left(x_{j}-y_{j}\right)^{2}\right)^{1 / 2}$ and $\mu$ equals Lebesgue measure;

(2) $X=\mathbf{R}^{n}, d(x, y)=\sum_{j=1}^{n}\left|x_{j}-y_{j}\right|^{\alpha_{j}}$, where $\alpha_{1}, \alpha_{2}, \ldots, \alpha_{n}$ are positive numbers, not all equal, and $\mu$ is Lebesgue measure (we shall refer to such distances as nonisotropic);

(3) Example (1) can be further generalized by letting $X$ and $d$ be as in (2) and $\mu$ a measure for which (2.1) holds. An important class of such measures is obtained by letting $d \mu(x)=[d(x, 0)]^{\beta} d x, \beta>0$, where $d x$ is the element of Lebesgue measure;

(4) $X=[0,1), \mu$ is Lebesgue measure and $d(x, y)$ is the length of the smallest dyadic interval containing $x$ and $y$ (we consider the intervals to be closed on the left and open on the right);

(5) $X=[-1,1], \mu$ is Lebesgue measure and $d \mu(x)=(1-x)^{\alpha}(1+x)^{\beta} d x$, where $\alpha, \beta>-1$ (this space is associated with the study of Jacobi polynomials);

(6) $X=\mathbf{R}_{+}=\{r \in \mathbf{R} ; r \geqslant 0\}, d \mu(r)=r^{n-1} d r, d$ is the usual distance (this space is important for the study of radial functions in $\mathbf{R}^{n}$ );

(7) Compact Riemannian manifolds with natural distances and measures furnish us with a wide variety of these spaces. Particular examples are compact Lie groups $G$ and the homogeneous spaces $G / K$ obtained from the closed subgroups of $G$. We shall study in detail the case of the surface $\Sigma_{n-1}$ of the unit sphere in $\mathbf{R}^{n}$ (which can be identified with $S O(n) / S O(n-1)$ ). The special case when $n=4$ is especially interesting since $\Sigma_{3}$ can be identified with $S U(2)$ (see [17]). For $\Sigma_{n-1}, d(x, y)=|x-y|$ and $\mu$ is the Lebesgue measure;

(8) $X$ is the boundary of a Lipschitz domain in $\mathbf{R}^{n}, \mu$ is harmonic measure and $d$ is Euclidean distance;

(9) When $X$ is the boundary of a smooth and bounded pseudo-convex domain in $\mathbf{C}^{n}$ one can introduce a nonisotropic quasi-distance that is related to the complex structure (see [42], [56] and example (13)) in such a way that we obtain a space of homogeneous type by using the Lebesgue surface measure. A concrete example that will be studied here is the surface of the unit sphere $\Sigma_{2 n-1}=\left\{z \in \mathbf{C}^{n}: z \cdot \bar{z}=\sum_{j=1}^{n} z_{j} \bar{z}_{j}=1\right\}$. The nonisotropic distance is given by $d(z, w)=|1-z \cdot \bar{w}|^{1 / 2}=\left|1-\sum_{j=1}^{n} z_{j} \bar{w}_{j}\right|^{1 / 2}$ (this is not equivalent to the distance given in the seventh example);

(10) Similarly, a quasi-distance can be introduced on the closure of a bounded, smooth pseudo-convex domain in order to obtain an example of one of our spaces (again, see [56] and example (13)). In the solid unit ball $B_{2 n}=\left\{z \in \mathbf{C}^{n}:|z|^{2}=z \cdot \bar{z} \leqslant 1\right\}$ the quasi-distance can be expressed in the form 


$$
d(z, w)=|| z|-| w||+\frac{|| z|| w|-\bar{z} \cdot w|}{|z||w|}
$$

when $z$ and $w$ are close (in the Euclidean sense) to the boundary. We shall consider this situation in greater detail later when we discuss the Bergman kernel associated with $B_{2 n}$;

(11) Other nonisotropic distances, besides those given in the second example, arise naturally on Euclidean spaces. For each $x=\left(x_{1}, x_{2}, x_{3}\right) \in \mathbf{R}^{3}$ consider the matrix

$$
\left(\begin{array}{ccc}
1 & x_{1} & x_{3}+\left(x_{1} x_{2} / 2\right) \\
0 & 1 & x_{2} \\
0 & 0 & 1
\end{array}\right)
$$

which we shall also denote by $x$. Matrix multiplication gives us a group structure on $\mathbf{R}^{3}$ and Haar measure coincides with Lebesgue measure. The reader can verify that the product of $x=\left(x_{1}, x_{2}, x_{3}\right)$ and $y=\left(y_{1}, y_{2}, y_{3}\right)$ is the point $\left(x_{1}+y_{1}, x_{2}+y_{2}, x_{3}+y_{3}+\left(x_{1} y_{2}-x_{2} y_{1}\right) / 2\right)$. The dilations $T_{\lambda} x$ $=\left(\lambda x_{1}, \lambda x_{2}, \lambda^{2} x_{3}\right), \lambda \in \mathbf{R}_{+}$, obviously preserve this operation; in fact they are automorphisms of this group of $3 \times 3$ matrices. In studying operators that commute with these automorphisms it is useful to employ the quasi-distance induced by the "norm" $\|x\|=\left|x_{1}\right|+\left|x_{2}\right|+\left|x_{3}\right|^{1 / 2}$. That is,

$$
d(x, y)=\left\|x y^{-1}\right\|=\left|x_{1}-y_{1}\right|+\left|x_{2}-y_{2}\right|+\left|x_{3}-y_{3}+\left(y_{1} x_{2}-x_{1} y_{2}\right) / 2\right|^{1 / 2} .
$$

This usefulness stems from the fact that $d$ is homogeneous with respect to $T_{\lambda}$; that is, $d\left(T_{\lambda} x, T_{\lambda} y\right)=\lambda d(x, y)$ for all $\mathbf{R}_{+}$. This is just a special case of a class of groups having such dilations (see [40]). This group is an example of a Heisenberg group. It can also be identified with the boundary of a "generalized half-space" that is the image of the ball $B_{4} \subset C^{2}$ under an appropriate Cayley transform (see [42]).

(12) Another class of examples is obtained if $X$ is a locally compact group for which there exists a countable base $\left\{U_{j}\right\}$ of open neighborhoods of the identity $1, j=0, \pm 1, \pm 2, \ldots$ (if $G$ is compact we consider only the indices $j \leqslant 0$ ) satisfying (a) $U_{j}=U_{j}^{-1}$, (b) $U_{j} U_{j} \subset U_{j+1}$, (c) $0<\mu\left(U_{j+1}\right)<c \mu\left(U_{j}\right)$, where $\mu$ is Haar measure and $c$ is a constant independent of $j$, (d) $\cup_{j} U_{j}=X$. We can then introduce a quasi-distance having the property that the solid spheres of radius $r>0$ have a measure that is essentially $r$. This is done in the following way: We first define a distance $m$ from 1 by letting $m(x)$ $=\inf \left\{\mu\left(U_{j}\right): x \in U_{j}\right\}$; the desired quasi-distance is then $d_{m}(x, y)=m\left(x y^{-1}\right)$ (see [17, p. 78], for a proof of this fact). If $X$ is the group of $3 \times 3$ matrices we considered in example (11) we obtain a countable base of open neighborhoods of the identity by letting $U_{j}=T_{2^{j}} B^{0}, j=0, \pm 1, \pm 2, \ldots$, where $B^{0}$ is the interior of the ordinary solid sphere of radius 1 in $\mathbf{R}^{3}$. The distance $d_{m}$ is then equivalent to the distance $d$ introduced in the previous example. Another interesting special case is furnished by the integers (or, more generally, by the group $\mathbf{Z}^{n}$ of lattice points in $\mathbf{R}^{n}$ ). Observe that in this case we have no differentiable structure. A similar situation arises when $X$ is the field of $p$-adic numbers. For example, we discuss briefly the 3-adic field $Q_{3}$. Formally the 
nonzero elements of $Q_{3}$ are represented by the sums $x=\sum_{l=k}^{\infty} a_{l} 3^{l}$, where $a_{l}=0,1$ or 2 , with $a_{k} \neq 0$. In this case the norm of $x$ is denoted by $\|x\|$ and is equal to $3^{-k}$. A neighborhood system for the origin is given by the "fractional ideals" $P^{k}=\left\{x \in Q_{3}:\|x\| \leqslant 3^{-k}\right\} . P^{0} \equiv R$ is the ring of integers in $Q_{3}$. If Haar measure (with respect to addition) $\mu$ is normalized so that $\mu(R)=1$ then it can be shown that $\mu\left(P^{k}\right)=3^{-k}$. It follows that $U_{j}=P^{-j}$ satisfy properties (a), (b), (c) and (d).

(13) In most of the examples we have given $X$ was either a group or had associated with it a natural group acting on it. A general method for obtaining spaces of homogeneous type that does not involve a group structure is the following. (Actually we can define the concept of a space of homogeneous type by this method.) Let $X$ be a topological space on which there is defined a Borel measure $\mu$. Suppose that at each point $x \in X$ there exists a basis of open neighborhoods $\left\{U_{x}^{\alpha}\right\}, \alpha \in \mathfrak{A}$, such that (a) $\cup_{\alpha} U_{x}^{\alpha}=X$, (b) $\cap_{\alpha} U_{x}^{\alpha}$ $=\{x\}$, (c) either: $U_{x}^{\alpha} \subset U_{x}^{\beta}$ or $U_{x}^{\beta} \subset U_{x}^{\alpha}$, (d) there exists a constant $c$ such that if $y \in U_{x}^{\alpha}$ then there exists $\beta \in \mathfrak{A}$ such that $U_{x}^{\alpha} \subset U_{y}^{\beta}$ and $\mu\left(U_{y}^{\beta}\right)$ $\leqslant c \mu\left(U_{x}^{\alpha}\right)$. It is easy to see that the spheres of a space of homogeneous type satisfy these properties. An appropriate quasi-distance $d$ is obtained by letting

$$
d(x, y)=\inf \left\{\mu\left(U_{x}^{\alpha}\right): y \in U_{x}^{\alpha}\right\}+\inf \left\{\mu\left(U_{y}^{\beta}\right): x \in U_{y}^{\beta}\right\}
$$

for all $x, y \in X$. If, for $r>0$, there exist neighborhoods $U_{x}^{\alpha} \subset U_{x}^{\beta}$ such that $c_{1} r \leqslant \mu\left(U_{x}^{\alpha}\right) \leqslant \mu\left(U_{x}^{\beta}\right) \leqslant c_{2} r$, where $c_{1}, c_{2}$ are independent of $x$ and $r$, then the spheres $B_{r}(x)$ defined by the quasi-distance $d$ have measure comparable to $r$. This situation occurs often and provides a technically convenient fact enabling one to obtain certain normalizations independent of the dimension.

When $X$ is the boundary of a smooth and bounded strongly pseudo-convex domain in $\mathbf{C}^{n}$ one can construct a quasi-distance by this method. A system of open neighborhoods at a point $x$ can be obtained by considering the surface of the osculating sphere $\Sigma$ at $x$ : The distance introduced in example (9) gives us a natural basis of open neighborhoods of $x$ as a point of $\Sigma$. These neighborhoods can then be projected onto $X$ and they, in turn, can be used to define a quasi-distance by the method we have just described.

If $\mathscr{D}$ is a bounded domain in $\mathbf{C}^{n}$ with smooth boundary $\partial \mathscr{D}$ define a smooth mapping $x \rightarrow \bar{x}$ of $\bar{D}$ to $\partial \mathcal{D}$ with the property that whenever $x$ is close to $\partial \mathcal{D}$ then $\bar{x}$ is the normal projection of $x$ to $\partial \mathscr{D}$. Let $\nu_{\bar{x}}$ be the unit vector in the direction of the outward normal to $\partial D^{2}$ at $\bar{x}$. Denote by $T_{x}^{2}$ the 1-dimensional complex vector space generated by $\nu_{\bar{x}}$. Let $T_{x}^{1}$ be the orthogonal complement of $T_{x}^{2}$. The sets

$$
U_{x}^{r}=\left\{z \in \mathscr{D}: z=x+z_{1}+z_{2}, z_{1} \in T_{x}^{1}, z_{2} \in T_{x}^{2},\left|z_{1}\right|^{2}+\left|z_{2}\right|<r\right\},
$$

$r>0$, form a system of neighborhoods at $x$ satisfying (a), (b), (c) and (d). ${ }^{19}$

19 The pseudo-convexity assumption is not necessary when we consider boundaries of smooth domains in $\mathbf{C}^{n}$. In fact, if we restrict the quasi-metric just defined on $\bar{D}$ to $\partial \mathcal{D}$ we obtain a distance that is equivalent to the one defined in the previous paragraph. We can make use of pseudoconvexity if we wish to avail ourselves of certain estimates for Szegö and Bergman kernels in order to study the operators they define on $H^{p}$ spaces and $L^{p}$ spaces associated with these domains (see [31], [56]). 
Before discussing the $H^{p}$-space theory associated with a space of homogeneous type we make some additional bibliographical comments. As we mentioned at the beginning of this section, spaces of homogeneous type were introduced in [12], [17] as a geometric setting for the basic real variable theory of singular integrals; at the same time Korányi and Vági [42] and N. Rivière [49] independently introduced this theory in the setting of example (12). The basic motivating examples were (9) and (11). The quasi-distances for pseudoconvex domains in $\mathbf{C}^{n}$ were given by Stein [56] who also proved that they give a space of homogeneous type. Various generalizations of these distances are in Rothschild and Stein [50].

The example discussed in the last part of (12) is a particular case of the martingale setup for $H^{p}$. It is in this setting that the atomic characterization of the $H^{p}$ space was obtained first by C. Herz [34]. For a discussion of $B M O$ and $H^{1}$ for martingales see Garsia's book Martingale inequalities, Benjamin, 1973.

We are now ready to introduce the Hardy spaces associated with a space of homogeneous type $X$. If $0<p<q, p \leqslant 1 \leqslant q \leqslant \infty$, we say that a function $a(x)$ is a $(p, q)$-atom if

(i) the support of $a$ is contained in a sphere $B_{r}\left(x_{0}\right)$;

(ii) $\left\{\left[1 / \mu\left(B_{r}\left(x_{0}\right)\right)\right] \int|a(x)|^{q} d \mu(x)\right\}^{1 / q} \leqslant\left\{\mu\left(B_{r}\left(x_{0}\right)\right)\right\}^{-1 / p}$;

(iii) $\int a(x) d \mu(x)=0$.

In case $\mu(X)<\infty$ the constant function having value $[\mu(X)]^{-1 / p}$ is also considered to be an atom. (In order to simplify certain calculations we shall henceforth assume $\mu(X)=1$ whenever $X$ has finite measure.) Observe that a $(p, q)$-atom is in $L^{1}(X, \mu)$ and is normalized in such a way that its $L^{p}(X, \mu)$ norm does not exceed 1 .

Recall that when we discussed $H^{p}$ spaces associated with $T$ or $\mathbf{R}$, when $p<1$, we pointed out that these were not spaces of functions defined on $T$ or R. In fact we considered the elements of $H^{p}$ to be distributions. In the present setting we are, again, forced to introduce an appropriate space of linear functionals in order to define the Hardy spaces. In order to do this we introduce the Lipschitz (or Hölder) spaces $\mathfrak{L}_{\alpha}, \alpha>0$, consisting of those functions $l$ on $X$ for which

$$
|l(x)-l(y)| \leqslant C[\mu(S)]^{\alpha},
$$

where $S$ is any sphere containing both $x$ and $y$ and $C$ depends only on $l .^{20}$ Let $\mathfrak{R}^{(\alpha)}(l)$ be the infimum of all $C$ for which (2.2) holds. If we define

${ }^{20}$ Unless $\alpha$ is sufficiently small it can happen that the only functions satisfying (2.2) are the constants. This is true for $T$ and $\mathbf{R}$ if $\alpha>1$. On the other hand if $X$ is the space described in example (4) (or is a p-adic field) any finite sum $\sum a_{i} \chi_{S_{i}}$, where the $\chi_{S_{i}}$ 's are characteristic functions of disjoint spheres, belongs to $\mathscr{L}_{\alpha}$ for all $\alpha>0$. In any case, for each of our examples, there is an interval of positive $\alpha$ 's where $\mathfrak{L}_{\alpha}$ is nontrivial. Condition (2.2) can also be written in the more familiar form $|l(x)-l(y)| \leqslant C m(x, y)^{\alpha}$ if $m$ is a quasi-distance such that the spheres of radius $r$ have measure comparable to $r$ (this can be done quite generally; see p. 78 of [17]). When $\mu(X)=\infty, \mathfrak{L}_{\alpha}$ really consists of equivalence classes of such functions: $l_{1}$ and $l_{2}$ are equivalent if $l_{1}-l_{2}$ is constant. We will consider this point in greater detail in $\$ 3$. 


$$
\|l\|^{(\alpha)}= \begin{cases}\mathfrak{N}^{(\alpha)}(l), & \text { if } \mu(X)=\infty, \\ \mathfrak{N}^{(\alpha)}(l)+\left|\int_{X} l(x) d \mu(x)\right|, & \text { if } \mu(X)=1,\end{cases}
$$

then $\|\cdot\|^{(\alpha)}$ is a norm. A straightforward argument shows that $\mathfrak{L}_{\alpha}$, so normed, is a Banach space.

If $a$ is a $(p, q)$-atom, $0<p<1$ and $\alpha=1 / p-1$, then using (i), (iii), (2.2) and (ii), in this order, we have

$$
\begin{aligned}
\left|\int_{X} a l d \mu\right| & =\left|\int_{B_{r}\left(x_{0}\right)} a l d \mu\right|=\left|\int_{B_{r}\left(x_{0}\right)} a(x)\left[l(x)-l\left(x_{0}\right)\right] d \mu(x)\right| \\
& \leqslant\|l\|^{(\alpha)}\left[\mu\left(B_{r}\left(x_{0}\right)\right)\right]^{1 / p-1} \int_{B_{r}\left(x_{0}\right)}|a(x)| d \mu(x) \leqslant\|l\|^{(\alpha)} .
\end{aligned}
$$

That is, the mapping $l \rightarrow \int_{X}$ ald $d \mu$ is a bounded linear functional on $\mathfrak{L}_{\alpha}$ with norm not exceeding 1 .

We now define the space $H^{p, q}$, for $0<p<1 \leqslant q$, to be the subspace of the dual $\mathfrak{L}_{\alpha}^{*}$ of $\mathfrak{L}_{\alpha}$, where $\alpha=1 / p-1$, consisting of those linear functionals admitting an atomic decomposition

$$
h=\sum_{j=0}^{\infty} \lambda_{j} a_{j}
$$

where the $a_{j}$ 's are $(p, q)$-atoms, $\Sigma_{0}^{\infty}\left|\lambda_{j}\right|^{p}<\infty$. (This last inequality implies that the series in (2.3) converges in the norms of $\mathfrak{L}_{\alpha}^{*}$ and we consider $h$ to be the limit, in this norm, of the partial sums of this series.) The infimum of the numbers $\Sigma_{0}^{\infty}\left|\lambda_{j}\right|^{p}$ taken over all such representations of $h$ will be denoted by the symbol $\|h\|_{p, q}$. The mapping $h \rightarrow\|h\|_{p, q}$ is clearly not a norm (it is homogeneous of order $p<1)$; But $d_{p, q}(h, g)=\|h-g\|_{p, q}$ is a metric. When $p=1<q \leqslant \infty$ the sums (2.3) are convergent in the $L^{1}$ norm whenever $\sum_{0}^{\infty}\left|\lambda_{j}\right|<\infty$ and the $a_{j}$ 's are $(1, q)$-atoms; hence, they define a subspace $H^{1, q}$ of $L^{1}(X)$. In this case the mapping $h \rightarrow\|h\|_{1, q}$ is a norm.

A straightforward argument shows that $H^{p, q}$ is complete. In particular $H^{1, q}$ is a Banach space. It also follows easily from the above definitions that

$$
H^{p, \infty} \subset H^{p, q_{2}} \subset H^{p, q_{1}}
$$

whenever $1<q_{1}<q_{2}<\infty$ and $0<p \leqslant 1$ ( $q_{1}$ may equal 1 when $\left.p<1\right)$. A basic result concerning these spaces is that the converses of the set-theoretic inclusions (2.4) hold:

Theorem A. $H^{p, q}=H^{p, \infty}$ whenever $p<q<\infty$. Moreover, the metrics $d_{p, q}$ and $d_{p, \infty}$ are equivalent; that is, there exists a constant $c$ (depending on $p$ and q) such that

$$
d_{p, q} \leqslant d_{p, \infty} \leqslant c d_{p, q}
$$

The proof of this theorem is rather technical. In order not to disrupt the flow of this presentation we shall give it later. 
Theorem A enables us to define the space $H^{p}=H^{p}(X)$ for $p \leqslant 1$ to be any one of the spaces $H^{p, q}$ for $p<q \leqslant \infty, 1 \leqslant q$. Any one of the metrics $d_{p, q}$ can be used in order to turn $H^{p}$ into a topological space. It is often useful to choose the space $H^{p, 2}$ and the metric $d_{p, 2}$ to work with when the space $X$ has a "Fourier transform" associated with it, since the $(p, 2)$-atoms are $L^{2}$. functions.

In order to characterize the dual of $H^{1}(X)$ we introduce the function spaces $B M O_{q}^{21}$ : a function $l$ on $X$ is said to belong to $B M O_{q}$ provided it is locally in $L^{q}(X)$ and

$$
\left[\frac{1}{\mu(S)} \int_{S}\left|l(x)-m_{S}(l)\right|^{q} d \mu(x)\right]^{1 / q} \leqslant C
$$

for all spheres $S$, where

$$
m_{S}(l)=\frac{1}{\mu(S)} \int_{S} l(y) d \mu(y)
$$

and $C$ is independent of $S$. Let $\mathfrak{R}_{q}(l)$ denote the infimum of all constants $C$ for which (2.5) holds. We can then introduce a "norm" on $B M O_{q}$ by defining

$$
\|l\|^{(q)}=\|l\|_{B M O}^{(q)}= \begin{cases}\mathfrak{N}_{q}(l), & \text { if } \mu(X)=\infty, \\ \mathfrak{N}_{q}(l)+\left|\int_{X} l(x) d \mu(x)\right|, & \text { if } \mu(X)=1 .\end{cases}
$$

When the measure of $X$ is finite, $B M O_{q}$, together with this norm, is a Banach space. If $\mu(X)=\infty$ then we consider the set of equivalence classes of functions defined by the relation " $l_{1}$ and $l_{2}$ in $B M O_{q}$ are equivalent if and only if $l_{1}-l_{2}$ is constant". If $l_{1}$ and $l_{2}$ are equivalent then, clearly, $\left\|l_{1}\right\|^{(q)}=\left\|l_{2}\right\|^{(q)}$. Thus, we can define the norm of each equivalence class to be the norm of any of its members and we obtain a Banach space which we also denote by $B M O_{q}$ (as is usually done when dealing with $L^{p}$-spaces we call the members of $B M O_{q}$ "functions" even when we really are talking about equivalence classes).

We shall show (in §3)

THeORem B. If $p<1$ and $\alpha=(1 / p)-1$ then $\mathfrak{Q}_{\alpha}$ is the dual of $H^{p}(X)$. That is, each continuous linear functional on $H^{p}$ is a mapping of the form $h$ $\rightarrow \sum \lambda_{j} \int a_{j} l d \mu$, where $l \in \mathfrak{L}_{\alpha}$ and $h=\sum \lambda_{j} a_{j} \in H^{p}($ see (2.3)). If $p=1$ then $B M O$ is the dual of $H^{1}(X)$. More precisely, if $h=\sum \lambda_{j} a_{j} \in H^{1}$ then

$$
\lim _{n \rightarrow \infty} \sum_{1}^{n} \lambda_{j} \int l a_{j} d \mu
$$

is a well-defined continuous linear functional $h \rightarrow\langle h, l\rangle$, for each $l \in B M O$, whose norm is equivalent to $\|l\|_{B M O}$; moreover, each continuous linear functional on $H^{1}$ has this form.

In the proof of Theorem $B$ it will be shown that $B M O_{q_{1}}$ and $B M O_{q_{2}}$,

21 "BMO" stands for bounded mean oscillation. These functions were introduced by John and Nirenberg [38]. We shall of ten write $B M O$ instead of $B M O_{1}$. 
$1 \leqslant q_{1}, q_{2}<\infty$, are equal as vector spaces and the norms \|\|$\left(q_{1}\right)$ and \|\|$\left(q_{2}\right)$ are equivalent. ${ }^{22}$ This will follow from the fact that $B M O_{q^{\prime}}$ characterizes $\left(H^{1, q}\right)^{*}$.

We introduced the notion of a molecule in the first section and showed how molecules can be used in order to obtain multiplier theorems. This notion extends to spaces of homogeneous type and, as we shall see, is quite useful for studying operators associated with some of the particular spaces we have described above. Let $\varepsilon>0$. We say that $M$ is an $\varepsilon$-molecule for $H^{1}=H^{1}(X)$ centered at $x_{0}$ if and only if

$$
\left\{\int_{X}|M(x)|^{2} d \mu(x)\right\}\left\{\int_{X}|M(x)|^{2}\left[m\left(x, x_{0}\right)\right]^{1+\varepsilon} d \mu(x)\right\}^{1 / \varepsilon} \leqslant 1,
$$

where $m\left(x, x_{0}\right)$ is the infimum of the measures of the spheres containing both $x$ and $x_{0}$, and

$$
\int_{X} M(x) d \mu(x)=0
$$

The function $m\left(x, x_{0}\right)$, defined on $X \times X$, will be called the measure distance. It can be shown quite generally that it is, indeed, a quasi-metric such that, if we consider it together with $X$ and $\mu$, we have a space of homogeneous type that is "equivalent" to $(X, \mu, d)$ in particular, the spaces $H^{p}(X)$ defined in terms of $d$ are the same as those defined in terms of $m$. Assuming this to be the case we shall now show

THEOREM C. If $M$ is a molecule for $H^{1}$ centered at $x_{0}$ then $M \in H^{1}$. Moreover, $\|M\|_{H^{1}}$ depends only on the constant $\varepsilon$ in (2.7).

The proof of this result illustrates again the importance of Theorem A since we shall show that $M$ is decomposable into a sum of $(1,2)$ - and $(1, \infty)$-atoms. To fix our ideas let us assume $X$ has infinite measure and that if $B_{r, m}\left(x_{0}\right)$ $=\left\{x: m\left(x, x_{0}\right)<r\right\}$ then $\mu\left(B_{r, m}\left(x_{0}\right)\right)$ is between $C_{1} r$ and $C_{2} r$, where $0<C_{1}$ $<C_{2}$ are constants independent of $x_{0}$ and $r$. We write $\mu\left(B_{r, m}\left(x_{0}\right)\right) \sim r$ to denote this relation between the radius of a sphere and its measure. ${ }^{23}$

Set $\sigma=\|M\|_{2}^{-2}$, let $\chi_{0}$ denote the characteristic function of $B_{\sigma}\left(x_{0}\right)$ $\equiv B_{\sigma, m}\left(x_{0}\right)$ and, for $i \geqslant 1$, let $\chi_{i}$ be the characteristic function of $\left\{x: \sigma 2^{i-1}\right.$ $\left.\leqslant m\left(x, x_{0}\right)<\sigma 2^{i}\right\}$. Define $M_{i}$ to be the function $M \chi_{i}-\left(\left(1 / \int \chi_{i}\right) \int M \chi_{i} d \mu\right)$ for $i=0,1,2, \ldots$ We shall show that (up to an unimportant multiplicative constant) $2^{i(\varepsilon / 2)} M_{i}$ is a $(1,2)$-atom. Clearly, $\int M_{i} d \mu=0$ for $i \geqslant 0$. Moreover,

$$
\left(\frac{1}{\sigma} \int\left|M_{0}\right|^{2} d \mu\right)^{1 / 2} \leqslant\left(\frac{1}{\sigma} \int_{B_{\sigma}\left(x_{0}\right)}|M|^{2} d \mu\right)^{1 / 2}+\left(\frac{1}{\sigma} \int_{B_{\sigma}\left(x_{0}\right)}|M| d \mu\right) \leqslant \frac{2}{\sigma} .
$$

22 This last assertion extends only part of a result of John and Nirenberg [38]. They show that BMO is equivalent to "exponential BMO" as well. Their proof can be adapted to spaces of homogeneous type.

23 It can be shown that, in general, $\mu\left(B_{r, m}\left(x_{0}\right)\right)$ is of the same order of magnitude as $r$ provided we take into account certain obvious nonessential difficulties (if $\mu(X)<\infty$, we cannot allow $r$ to be too large; if $\mu\left(\left\{x_{0}\right\}\right)>0$ we cannot allow $r$ to be too small). These are technical points that we do not wish to elaborate on at the present time. 
Thus, $(1 / 2) M_{0}$ is a $(1,2)$-atom. If $i \geqslant 1$ we make the following estimate in which (2.7) is used:

$$
\begin{aligned}
\left(\int\left|M_{i}\right|^{2} d \mu\right)^{1 / 2} & \leqslant 2\left(\int|M|^{2} \chi_{i}\right)^{1 / 2} \\
& =2\left(\int|M(x)|^{2}\left[m\left(x, x_{0}\right)\right]^{1+\varepsilon}\left[m\left(x, x_{0}\right)\right]^{-1-\varepsilon} \chi_{i}(x) d \mu(x)\right)^{1 / 2} \\
& \leqslant\left(\sigma 2^{i-1}\right)^{-(1+\varepsilon) / 2} C^{\varepsilon / 2} \sigma^{\varepsilon / 2}=C^{\prime}\left(2^{i} \sigma\right)^{-1 / 2}\left(2^{i \varepsilon}\right)^{-1 / 2} .
\end{aligned}
$$

It follows that

$$
\left(\int\left|M_{i}\right|^{2} \frac{d \mu}{\mu\left(B_{2 i}\left(x_{0}\right)\right)}\right)^{1 / 2} \leqslant \text { (const) } 2^{-i} \sigma 2^{-i \varepsilon / 2}
$$

and, thus, $2^{i \varepsilon / 2} M_{i}$ is a constant multiple of an $(1,2)$-atom. We have $M=\sum_{i=0}^{\infty} M_{i}+\sum_{i=0}^{\infty} m_{i} \chi_{i}^{\prime}$, where $m_{i}=\int M \chi_{i} d \mu$ and $\chi_{i}^{\prime}=\chi_{i} / \int \chi_{i}$. The argument we just gave shows that $\sum_{i=0}^{\infty} M_{i} \in H^{1}$ and the $H^{1}$ norm of this function is, up to a constant, less than or equal to $2^{\varepsilon / 2} /\left(2^{\varepsilon / 2}-1\right)$. Let $N_{j}=\sum_{i=j}^{\infty} m_{i}$. Then, summing by parts and using $\sum_{0}^{\infty} m_{i}=\int M d \mu=0$ we have

$$
\sum_{i=0}^{\infty} m_{i} \chi_{i}^{\prime}=\sum_{j=0}^{\infty}\left(N_{j}-N_{j+1}\right) \chi_{j}^{\prime}=\sum_{j=0}^{\infty} N_{j+1}\left(\chi_{j+1}^{\prime}-\chi_{j}^{\prime}\right) .
$$

Since $\int \chi_{j}^{\prime} d \mu=1$ for all $j$ we have $\int\left(\chi_{j+1}^{\prime}-\chi_{j}^{\prime}\right) d \mu=0$. Moreover, the support of $\chi_{j+1}^{\prime}-\chi_{j}^{\prime}$ lies within $B_{2^{j+1} \sigma}$ which has measure $\sim 2^{j+1} \sigma$. From the definition of $\chi_{j}^{\prime}$ we also have $\left|\chi_{j+1}^{\prime}-\chi_{j}^{\prime}\right| \leqslant 1 / \int \chi_{j+1}+1 / \int \chi_{j} \leqslant 2 / \int \chi_{j}$. Since $\mu\left(B_{2^{j} \sigma}\left(x_{0}\right)\right) \sim \sigma^{2^{j}}$ it follows that $\int \chi_{j} \sim \mu\left(B_{2^{j} \sigma}\left(x_{0}\right)\right)-\mu\left(B_{2^{j-1} \sigma}\left(x_{0}\right)\right) \sim 2^{j} \sigma$ $-2^{j-1} \sigma=2^{j-1} \sigma=2^{j \sigma} / 2 .{ }^{24}$ This shows that, up to a multiplicative constant $\chi_{j+1}^{\prime}-\chi_{j}^{\prime}$ is a $(1, \infty)$-atom. Furthermore, making use of (2.7) again we have

$$
\begin{aligned}
\left|N_{j}\right| & \leqslant \sum_{i=j}^{\infty} \int\left|M \chi_{i}\right| d \mu \lesssim \sum_{i=j}^{\infty}\left(\int|M|^{2} \chi_{i}\right)^{1 / 2}\left(\sigma 2^{j}\right)^{1 / 2} \\
& \left.\leqslant C \sum_{i=j}^{\infty} \dot{(} \sigma 2^{i}\right)^{-1 / 2} 2^{-i \varepsilon / 2}\left(\sigma 2^{i}\right)^{1 / 2}=C_{\varepsilon}\left(2^{-\varepsilon / 2}\right)^{j} .
\end{aligned}
$$

This shows that $\sum_{i=0}^{\infty} m_{i} \chi_{i}^{\prime}$ belongs to $H^{1}\left(=H^{1, \infty}\right)$ and, up to a multiplicative constant, its norm does not exceed $2^{\varepsilon / 2} /\left(2^{\varepsilon / 2}-1\right)$. This establishes Theorem C.

Before developing the general theory further let us make three observations. First, recall that immediately following (1.17) we pointed out that any linear operator mapping atoms into molecules satisfying (1.15) has a bounded extension mapping $H^{1}$ into itself. The argument we gave, however, used the fact that the conjugate function operator maps $H^{1}$ boundedly into itself.

24 Recall that the notation $\mu\left(B_{r}\left(x_{0}\right)\right) \sim r$ meant that for appropriate constants $0<C_{1}<C_{2}$ we have $C_{1} r \leqslant \mu\left(B_{r}\left(x_{0}\right)\right) \leqslant C_{2} r$. We are tacitly assuming that $2 C_{1}>C_{2}$. If this were not the case then $q C_{1}>C_{2}$ would hold for a sufficiently large $q$. The argument we are giving would still be valid if $q^{j}$ is used when $2^{j}$ occurs. 
Theorem $\mathrm{C}$ shows that this was not necessary and that, in general, a linear operator mapping atoms into molecules satisfying $(2.7)$ and $\left(2.7^{\prime}\right)$ has a bounded extension mapping $H^{1}$ into itself.

Secondly, it should be remarked that any $(1, \infty)$ - (or $(1,2)-$ ) atom is a molecule for $H^{1}$. If we define such an atom in terms of the measure distance this follows from a straightforward computation.

Thirdly, when we introduced inequality (1.17) we did so in order to indicate the role played by molecules for $\boldsymbol{H}^{p}$ for some values of $p$ less than 1 . This can be done in general. We leave it for the reader to verify that appropriate molecules for $\boldsymbol{H}^{p}$ can be defined and that the values of $\boldsymbol{p}$ for which this can be done depend on $\varepsilon$.

We shall now discuss two interpolation theorems for operators acting on $H^{p}(X)$ and $L^{q}(X)$. The first result is a Marcinkiewicz-type interpolation theorem (see [64]). In order to state it we need to introduce the notion of weaktype operators. Suppose $B$ is a map from a vector space into measurable functions on a measure space $(Y, v)$; we say that $B$ is sublinear provided $|B(f+g)| \leqslant|B f|+|B g|$ and $|B a f|=|a||B f|$ a.e. whenever $f$ and $g$ belong to the domain of $B$ and $a$ is a scalar. If $B$ is defined on $H^{p}(X)$, for some $p \leqslant 1$, we say that it is of weak-type $\left(H^{p}, p\right)$ provided $\nu(\{y \in Y:|(B f)(y)|>\lambda\})$ $\leqslant(M / \lambda)^{p}\|f\|_{H^{p}}$ for all $f \in H^{p}$, where $\|f\|_{H^{p}}$ denotes any one of the "norms" $\|f\|_{p, q}{ }^{25}$ If $B$ is defined on $L^{p}(X), 0<p<\infty$, we say that it is of weak-type $(p, p)$ provided $\nu(\{y \in Y:|(B f)(y)|>\lambda\}) \leqslant\left(M\|f\|_{p} / \lambda\right)^{p}$ for all $f \in L^{p}$; we define weak-type $(\infty, \infty)$ to be the same as boundedness: $\|B f\|_{\infty} \leqslant M\|f\|_{\infty}$ for all $f \in L^{\infty}(X)$. For $p$ fixed, the infimum of all constants $M$ for which any one of these inequalities hold (independently of $f$ ) is called the weak-type norm of $B$.

We can now announce the first interpolation theorem:

THeOREM D. Suppose $0<p_{1} \leqslant 1 \leqslant p_{2} \leqslant \infty, p_{1}<p_{2}$, and $B$ is a sublinear operator of weak-types $\left(H^{p_{1}}, p_{1}\right)$ and $\left(p_{2}, p_{2}\right)$ having weak-type norms $M_{1}$ and $M_{2}$. If $1<p<p_{2}$ then $B$ is defined on $L^{p}(X)$ and

$$
\|B f\|_{p} \leqslant M\|f\|_{p}
$$

where $M$ depends on $M_{1}, M_{2}, p_{1}$ and $p_{2}$ but is independent of $f \in L^{p}$. If $p_{1}<p \leqslant 1$ then $B$ is defined on $H^{p}(X)$ and

$$
\|B f\|_{p} \leqslant M\|f\|_{H^{p}}^{1 / p}
$$

where $M$ depends on $M_{1}, M_{2}, p_{1}$ and $p_{2}$ but is independent of $f \in H^{p}$.

This result is proved in the Ph.D. thesis of one of our students, R. Macias [45]. It was obtained in the classical situation by Igari [37] (for Riesz-systems when $p_{1}, p_{2} \geqslant 1$ ) and C. Fefferman, Rivière and Sagher [30].

The original theorem of Marcinkiewicz dealt only with $L^{p}$-spaces and was proved by truncating the function $f$ at heights $\lambda \in(0, \infty)$ and making

${ }^{25}$ Of course, when we vary $q$ the constant $M$ changes accordingly. The important fact is that \|\|$_{p, q_{1}}$ and \|\|$_{p, q_{2}}$ are equivalent. We could have defined the notion of weak type $\left(H^{p_{1}}, p_{2}\right)$ with $p_{1} \neq p_{2}$ as well. For simplicity we restrict ourselves to the case $p_{1}=p_{2}$. 
appropriate estimates. In our case a substitute for these truncations is obtained by a variant of the Calderón-Zygmund decomposition. We shall give more details about this proof in the next section.

The second interpolation theorem involves an adaptation of what is known as the "complex method" and extends a result on interpolation of analytic families of operators on classical $H^{p}$ spaces (see [58]). Before stating this theorem we need to make some definitions. Let $D=\{z=x+i y \in \mathbf{C}: 0$ $<x<1\}$. A complex-valued function $F$ defined on $\bar{D}$ is said to be of admissible growth if there exists a constant $a<\pi$ such that $e^{-a|y|} \log |F(z)|, z$ $=x+i y$, is uniformly bounded from above in the strip $D$. Let us first consider a space of homogeneous type $X$ such that $\mu(X)=\infty$. Suppose that, for each $z \in \bar{D}, B_{z}$ is a linear operator defined on $L^{\infty}(X)$-functions having bounded support and mean 0 (we shall denote this class of functions by $L_{0}^{\infty}(X)$ ); we assume that the range of $B_{z}$ consists of measurable functions on a measure space $(Y, \nu)$ and that $\left(B_{z} f\right) g \in L^{1}(Y)$ whenever $f \in L_{0}^{\infty}(X)$ and $g$ is a simple function on $Y$. The family $\left\{T_{z}\right\}$ is called an analytic family of operators on $\bar{D}$ provided $F(z)=\int_{Y}\left(T_{z} f\right) g d v$ is continuous on $\bar{D}$, analytic in $D$ and of admissible growth whenever $f \in L_{0}^{\infty}(X)$ and $g$ is a simple function on $Y$. If $X$ has finite measure we replace $L_{0}^{\infty}(X)$ by $L^{\infty}(X)$ in the definitions above.

THEOREM E. Suppose $\left\{B_{z}\right\}$ is an analytic family of operators satisfying

$$
\left\|B_{i y} f\right\|_{p_{0}} \leqslant A_{0}(y)\|f\|_{H_{p_{0}}}^{1 / p_{0}} \text { and }\left\|B_{1+i y} f\right\|_{p_{1}} \leqslant A_{1}(y)\|f\|_{p_{1}}
$$

for all $y \in(-\infty, \infty)$ and $f \in L_{0}^{\infty}(X)\left(f \in L^{\infty}(X)\right.$ if $\left.\mu(X)<\infty\right)$, where $\log A_{j}(y) \leqslant C_{j} e^{d_{j}|y|}, C_{j}>0, \pi>d_{j}>0, j=1,2$, and $p_{0} \leqslant 1 \leqslant p_{1}$. If $1 / p$ $=t / p_{0}+(1-t) / p_{1}<1(0<t<1)$ then

$$
\left\|B_{t} f\right\|_{p} \leqslant M\|f\|_{p}
$$

while, if $1 / p=t / p_{0}+(1-t) / p_{1} \geqslant 1 \quad(0<t<1)$,

$$
\left\|B_{t} f\right\|_{p} \leqslant M\|f\|_{H^{p}}^{1 / p}
$$

where $M$ depends on $C_{0}, C_{1}, d_{0}, d_{1}, p_{0}, p_{1}$ but is independent of $f$ $\in L_{0}^{\infty}(X)\left(L^{\infty}(X)\right.$ if $\left.\mu(X)<\infty\right)$.

The proof of this theorem (to be found in the thesis of R. Macias [45]) uses techniques developed by Stein and Fefferman (see [29]) in order to obtain inequality (2.8), and a modification of an argument found in [58] in order to obtain inequality (2.9). We shall give details of this proof in $\$ 3$.

We shall now illustrate how these results involving the atomic $H^{p}$ spaces we have introduced can be applied to problems in harmonic analysis associated with the spaces of homogeneous type we described above.

Let us begin with example (1) where $X=\mathbf{R}^{n}, \mu$ is Lebesgue measure and $d$ is Euclidean distance. In $\$ 1$ we discussed the Riesz transforms and some of their properties. These operators are particular examples of the convolution operator 


$$
\tilde{f}(x)=\lim _{\varepsilon \rightarrow 0+} \int_{\varepsilon<|y|<1 / \varepsilon} k(y) f(x-y) d y \equiv(k * f)(x),
$$

where

$$
k(y)=\Omega\left(y^{\prime}\right) /|y|^{n}, \quad y \neq 0, \quad y^{\prime}=y /|y|, \quad \Omega \in L^{1}\left(\Sigma_{n-1}\right)
$$

and

$$
\int_{\Sigma_{n-1}} \Omega\left(y^{\prime}\right) d y^{\prime}=0
$$

(here $d y^{\prime}$ denotes the element of Lebesgue surface measure). If $\Omega$ satisfies the Lipschitz condition $\left|\Omega\left(x^{\prime}\right)-\Omega\left(y^{\prime}\right)\right| \leqslant C^{\prime}\left|x^{\prime}-y^{\prime}\right|$ then the kernel $k$ satisfies inequality (1.36)

$$
|k(x-y)-k(x)| \leqslant C|y| /|x|^{n+1}
$$

for an appropriate constant $C$ whenever $2|y|<|x|$. But we showed above that this property and $L^{2}$-boundedness was all that we need in order to conclude that the operator defined by $(2.10)$ is bounded from $H^{1}$ into $L^{1}$ (or, for that matter, from $H^{p}$ for $n /(n+1)<p$ to $L^{p}$-see (1.35)). Theorem $\mathrm{D}$ can now be used to conclude that this operator is bounded on $L^{p}\left(\mathbf{R}^{n}\right)$ for $1<p \leqslant 2$ (since the adjoint of this operator is its negative, this operator is bounded on $L^{p}$ for $\left.1<p<\infty\right) .26$

It is not hard to show $f \rightarrow k * f$ maps $H^{1}$ boundedly into itself. Because of Theorem $\mathrm{C}$ it suffices to show that $\tilde{a}=k * a$ is a constant multiple of a molecule whenever $a$ is an atom. To see this let $a(x)$ be a $(1,2)$-atom supported in a sphere of radius $r$ about $x_{0} \in \mathbf{R}^{n}$. It is clear that $\tilde{a}$ has mean 0 . Since $a$ has mean 0 we have

$$
\tilde{a}(x) \equiv \lim _{\varepsilon \rightarrow 0} \int_{\varepsilon<|x-y|<1 / \varepsilon} k(x-y) a(y) d y=\int_{\mathbf{R}^{n}}\left[k(x-y)-k\left(x-x_{0}\right)\right] a(y) d y .
$$

Thus, using (2.11), we have, for $\left|x-x_{0}\right|>2 r$,

$$
\begin{aligned}
|\tilde{a}(x)| & \leqslant \int_{\left|y-x_{0}\right|<r} \frac{\left|y-x_{0}\right|}{\left|x-x_{0}\right|^{n+1}}|a(y)| d y \\
& \leqslant \frac{r}{\left|x-x_{0}\right|^{n+1}} \int|a(y)| d y \leqslant \frac{r}{\left|x-x_{0}\right|^{n+1}} .
\end{aligned}
$$

It follows immediately from property (ii) of a $(1,2)$-atom that $\int|a(x)|^{2} d x$ $\leqslant$ (const) $r^{-n}$. Making use of these estimates on $\tilde{a}(x)$ and $\|a\|_{2}^{2}$ as well as the $L^{2}$-boundedness of the operator $a \rightarrow \tilde{a}$ we have that a constant multiple of

$$
\left(\int|\tilde{a}(x)|^{2} d x\right)\left(\int|\tilde{a}(x)|^{2}\left|x-x_{0}\right|^{n+1} d x\right)^{n}
$$

26 The $L^{2}$-boundedness of such operators can be established easily by calculating the Fourier transform of $k * f($ see [57]). In other situations where the Fourier-transform is not available one can use a general method developed by Cotlar, Knapp and Stein that is adaptable to spaces of homogeneous type (see [40]). 
is less than or equal to

$$
\begin{aligned}
& r^{-n}\left[\left(\int_{\left|x-x_{0}\right|<2 r}|\tilde{a}(x)|^{2}\left|x-x_{0}\right|^{n+1} d x\right)^{n}\right. \\
& \left.\quad+\left(\int_{\left|x-x_{0}\right| \geqslant 2 r}|\tilde{a}(x)|^{2}\left|x-x_{0}\right|^{n+1} d x\right)^{n}\right] \\
& \leqslant r^{-n}\left(r^{n+1}\|\tilde{a}\|_{2}^{2}\right)^{n}+r^{-n}\left(\int_{\left|x-x_{0}\right| \geqslant 2 r}\left(\frac{r}{\left|x-x_{0}\right|^{n+1}}\right)^{2}\left|x-x_{0}\right|^{n+1} d x\right)^{n} \\
& \leqslant(\text { const }) r^{-n}\left(r^{n+1}\|a\|_{2}^{2}\right)^{n}+r^{-n} r^{2 n}\left(\int_{\left|x-x_{0}\right| \geqslant 2 r} \frac{d x}{\left|x-x_{0}\right|^{n+1}}\right)^{n} \\
& \leqslant \text { (const) }\left[r^{-n} r^{n}+r^{n} r^{-n}\right] .
\end{aligned}
$$

This shows that, up to a multiplicative constant independent of $a$,

$$
\left(\int|\tilde{a}(x)|^{2} d x\right)\left(\int|\tilde{a}(x)|^{2}\left(\left|x-x_{0}\right|^{n}\right)^{(1+(1 / n))} d x\right)^{n}
$$

satisfies condition (2.7) with $\varepsilon=1 / n$ (observe that $m\left(x, x_{0}\right)=\left|x-x_{0}\right|^{n}$ is a measure distance in this case).

Inequality (2.11) implies the weaker condition

$$
\int_{|x|>2|y|}|k(x-y)-k(x)| d x \leqslant C .
$$

In establishing (1.24) we gave an argument that is easily modified to show that this condition, together with the boundedness of $\hat{k}$, implies that the operator $f \rightarrow k * f$ maps $H^{1}$ boundedly into $L^{1}$. Both (2.11) and (2.12) have natural extensions to spaces of homogeneous type. In fact, it is easy to see that the argument we have just given shows that if an integral operator

$$
(B f)(x)=\int_{X} f(y) k(x, y) d \mu(y)
$$

is bounded on $L^{2}(X), B f$ has mean 0 when $f$ is an atom and has a kernel satisfying, for $m\left(x, y_{0}\right)>C m\left(y, y_{0}\right)$,

$$
\left|k(x, y)-k\left(x, y_{0}\right)\right| \leqslant C\left[\frac{m\left(y, y_{0}\right)}{m\left(x, y_{0}\right)}\right]^{\varepsilon} \frac{1}{m\left(x, y_{0}\right)}
$$

then it maps $H^{1}(X)$ boundedly into itself. (In fact it maps $H^{p}$ into itself for $p$ close enough to 1 .) If the kernel satisfies

$$
\int_{d\left(x, x_{0}\right)>C_{1} d\left(y, x_{0}\right)}\left|k(x, y)-k\left(x, x_{0}\right)\right| d \mu(x) \leqslant C_{2}
$$

and $B$ is bounded on $L^{2}(X)$ then $B$ maps $H^{1}$ boundedly into $L^{1}(X)$ with a norm that depends on the constants $C_{1}, C_{2}$ and the $L^{2}$-operator norm of $B$. This follows from a simple modification of the argument used to establish (1.24). 
We remark that molecules can be defined in terms of other norms besides the $L^{2}$ norm. For example, a function $M \in L^{1+\delta}, \delta>0$, satisfying

$$
\int M d \mu=0 \text { and }\left(\int|M|^{1+\delta} d \mu\right)^{1 / \delta}\left(\int|M(x)|^{1+\delta}\left[m\left(x, x_{0}\right)\right]^{\delta+\varepsilon} d \mu(x)\right)^{1 / \varepsilon} \leqslant 1
$$

can easily be shown to belong to $H^{1}$ (or, with a change in normalization, to $H^{p}$, for some $p<1$ ). Suppose

$$
(B f)(x)=\int k(x, y) f(y) d \mu(y)
$$

is a bounded operator on $L^{2}$, then a sufficient condition on $k(x, y)$ that implies that atoms are mapped by $B$ onto these (more general) molecules (and, thus, $B$ is a bounded transformation of $H^{p}$ into itself for some $p<1$ ) is

$$
\begin{gathered}
\int_{m\left(x, y_{0}\right)>C m\left(y, y_{0}\right)}\left|k(x, y)-k\left(x, y_{0}\right)\right|^{1+\delta}\left[m\left(x, y_{0}\right)\right]^{\delta+\varepsilon} d \mu(x) \\
\leqslant C\left[m\left(y, y_{0}\right)\right]^{\varepsilon} .
\end{gathered}
$$

This condition holds whenever (2.13) is valid and reduces to (2.14) if $\delta=\varepsilon=0$.

These observations illustrate how the atomic theory of $H^{p_{\text {-spaces }}}$ can be used to study certain Calderón-Zygmund singular integrals. In order to obtain the boundedness of these operators on $L^{p}\left(\mathbf{R}^{n}\right), 1<p<\infty$, much less stringent conditions are needed. In fact, if $\Omega$ is an odd function in $L^{1}\left(\Sigma_{n-1}\right)$ one obtains this boundedness (see [57]). When $\Omega$ is even we need to know more; Calderón-Zygmund [4] show that if $\Omega \in L \log L\left(\Sigma_{n-1}\right)$ then $B$ is bounded on $L^{p}\left(\mathbf{R}^{n}\right)$.

If $n=2$ then this boundedness follows from the assumption that $\Omega$ $\in H^{1}\left(\Sigma_{1}\right)=H^{1}(T)$ (see p. 160 of [21]). We shall now show that this class of operators together with the identity forms an algebra. Let us begin by characterizing the Fourier transforms of the operators having a kernel $k(y)=\Omega\left(y^{\prime}\right) /|y|^{2}$ with $\Omega \in H^{1}(T)$. In terms of polar coordinates any such operator has the form

$$
(B f)(x)=\lim _{\varepsilon \rightarrow 0+} \int_{\varepsilon}^{1 / \varepsilon} \int_{0}^{2 \pi} \frac{\Omega(\theta)}{r} f\left(x-r e^{i \theta}\right) d r d \theta .
$$

If we write $y=r e^{i \psi}$ then it can be shown (see p. 162 of [57]) that if $k_{j}\left(r e^{i \theta}\right)=\Omega_{j}(\theta) / r^{2}=e^{i j \theta} / r^{2}$ then

$$
\hat{k}_{j}(y)=\lim _{\varepsilon \rightarrow 0} \int_{\varepsilon}^{1 / \varepsilon} \int_{0}^{2 \pi} \frac{e^{i j \theta}}{r} e^{-i y \cdot r e^{i \theta}} d r d \theta=\frac{2 \pi}{|j|} e^{i j \psi_{i}-|j|} .
$$

It follows from Hardy's inequality (1.5) that if

$$
\Omega(\theta) \sim \sum_{j \neq 0} a_{j} e^{i j \theta}
$$

belongs to $H^{1}(T)$ then 


$$
\hat{k}(y)=2 \pi \sum_{j \neq 0} \frac{i^{-|j|} a_{j}}{|j|} e^{i j \psi}
$$

and this last series converges absolutely. Let

$$
\Omega=\Omega_{\mathrm{o}}+\Omega_{\mathrm{e}} \text { where }[\Omega(\theta+\pi)+\Omega(\theta)] / 2=\Omega_{\mathrm{e}}(\theta)
$$

( $\Omega_{\mathrm{e}}$ and $\Omega_{\mathrm{o}}$ are the even and odd parts of $\Omega$ as a function on $\mathbf{R}^{2}$ ). Then the function $\hat{k}\left(e^{i \psi}\right)=\hat{k}(y)$ has the property

$$
((d / d \psi) \hat{k})\left(e^{i \psi}\right)=2 \pi\left[\Omega_{\mathrm{o}}(\psi-\pi / 2)+\tilde{\Omega}_{\mathrm{e}}(\psi-\pi / 2)\right],
$$

where $\tilde{\Omega}_{\mathrm{e}}$ is the conjugate function of $\Omega_{\mathrm{e}}$ (if we consider the imaginary part $Q(\theta)$ of the kernel $\left(1+e^{i \theta}\right) /\left(1-e^{i \theta}\right)$ discussed in the paragraph following (1.25), then $\tilde{\Omega}_{\mathrm{e}}$ is the generalized convolution $\left.\Omega_{\mathrm{e}} * Q\right)$. It follows that $(d / d \psi) \hat{k}$ $\in H^{1}(T)$. Conversely, if $\hat{k}(y)$ is a function on $\mathbf{R}^{2}$ that is homogeneous of degree 0 and satisfies

$$
\frac{d}{d \psi} \hat{k} \in H^{1} \text { and } \int_{T} \hat{k}\left(e^{i \psi}\right) d \psi=0
$$

then there exists a function $k$ on $\mathbf{R}^{2}$ of the form $\Omega(\theta) / r^{2}$ with $\Omega \in H^{1}$ such that $\hat{k}$ is the Fourier transform of $k$.

We shall now show that the vector space generated by these operators together with the identity form an algebra by showing that $\hat{k} \hat{h}$ has a derivative (with respect to $\psi$ ) in $H^{1}$ whenever $\hat{h}$ and $\hat{k}$ are homogeneous of degree 0 and have derivatives in $H^{1}$. Suppose, then, that $\hat{h}\left(e^{i \psi}\right)=\sum \lambda_{j} A_{j}(\psi)$ and $\hat{k}\left(e^{i \psi}\right)$ $=\sum \mu_{l} B_{l}(\psi)$ where $A_{j}^{\prime}, B_{l}^{\prime}$ are atoms, $\Sigma\left|\lambda_{j}\right|<\infty$ and $\sum\left|\mu_{l}\right|<\infty$. Obviously, $\sum_{j, l}\left|\lambda_{j} \mu_{l}\right|<\infty$ and we only have to show that $(1 / 2)(A B)^{\prime}$ is an atom whenever $A^{\prime}$ and $B^{\prime}$ are nonconstant atoms. Suppose $A$ has a supporting interval $I$ and $B$ has a supporting interval $J$ (since the atoms have mean zero $A$ and $A^{\prime}$ have the same supporting interval). The support of $A B$ is contained in $I \cap J$. Moreover, $\left|(A B)^{\prime}\right|=\left|A^{\prime} B+A B^{\prime}\right| \leqslant 1 /|I|+1 /|J|$ (the absolute value of the integral of an atom is always less than or equal to 1). Thus, $\left|(A B)^{\prime}\right| \leqslant 2 /|I \cap J|$ and our claim is established.

It is not hard to show that in dimension $n>2$ the condition $\Omega \in H^{1}\left(\Sigma_{n-1}\right)$ implies the boundedness of the associated singular integral operator on $L^{p}\left(\mathbf{R}^{n}\right), 1<p<\infty$. It is natural to raise the question whether such operators, together with the identity, generate an algebra.

Let us now turn to example (2) in which we introduced nonisotropic distances on $\mathbf{R}^{n}$. We shall show how the theory we have described, applied to this situation, can be used in order to obtain a priori estimates for certain partial differential equations. Let us consider the special case of the heat equation

$$
\partial u / \partial x_{2}-\partial^{2} u / \partial x_{1}^{2}=g\left(x_{1}, x_{2}\right)
$$

where $x=\left(x_{1}, x_{2}\right) \in \mathbf{R}^{2}$. It will become clear that the arguments presented here apply to a wide variety of equations. Suppose $g \in L^{p}\left(\mathbf{R}^{2}\right)$, for an 
appropriate $p$, can we find a solution $u$, such that $\partial u / \partial x_{2}$ and $\partial^{2} u / \partial x_{1}^{2}$ also belong to $L^{p}\left(\mathbf{R}^{2}\right)$ ? By applying (formally) the Fourier transform we see that our equation is equivalent to

$$
\left(y_{1}^{2}+i y_{2}\right) \hat{u}\left(y_{1}, y_{2}\right)=\hat{g}\left(y_{1}, y_{2}\right)
$$

thus,

$$
\begin{aligned}
& \left(\frac{\partial u}{\partial x_{2}}\right)^{\wedge}\left(y_{1}, y_{2}\right)=\frac{i y_{2}}{y_{1}^{2}+i y_{2}} \hat{g}\left(y_{1}, y_{2}\right) \quad \text { and } \\
& \left(\frac{\partial^{2} u}{\partial x_{1}^{2}}\right)^{\wedge}\left(y_{1}, y_{2}\right)=\frac{-y_{1}^{2}}{y_{1}^{2}+i y_{2}} \hat{g}\left(y_{1}, y_{2}\right) .
\end{aligned}
$$

We are, therefore, led to consider the operator assigning to $g$ the function

$$
(M g)(x)=\left[\frac{i y_{2}}{y_{1}^{2}+i y_{2}} \hat{g}\left(y_{1}, y_{2}\right)\right]^{\nu}(x)
$$

(as well as the operator induced by the multiplier $\left.y_{1}^{2} /\left(y_{1}^{2}+i y_{2}\right)\right)$. $M$ is obviously a bounded operator on $L^{2}\left(\mathbf{R}^{2}\right)$. We claim that by choosing an appropriate distance on $\mathbf{R}^{2}$ we will find it easy to show that $M$ preserves the $H^{p}$ spaces associated with this distance, for certain values of $p$; consequently, $M$ is bounded on the corresponding Lipschitz spaces and the $L^{q}$ spaces for $1<q<\infty$.

A natural way for introducing this distance is obtained by considering the family of "dilations" on $\mathbf{R}^{2}$ mapping $x$ into $T_{\lambda} x=\left(\lambda^{1 / 3} x_{1}, \lambda^{2 / 3} x_{2}\right), \lambda>0$. Let us also write $\left(T_{\lambda} g\right)(x) \equiv g\left(T_{\lambda} x\right)$. It is then clear that $M\left(T_{\lambda} g\right)=T_{\lambda}(M g)$ for all $\lambda>0$. That is, the operators $M$ and $T_{\lambda}$ commute.

We now introduce the "norm" $\|x\|=\left|x_{1}\right|^{3}+\left|x_{2}\right|^{3 / 2}$ in order to obtain families of spheres in $\mathbf{R}^{2}$ that are invariant under the action of these dilations. Observe that

$$
\left\|T_{\lambda} x\right\|=\lambda\|x\|
$$

for all $\lambda>0$. By letting $B_{\lambda}=\left\{x \in \mathbf{R}^{2}:\|x\|<\lambda\right\}, \lambda>0$, we obtain the family of spheres about the origin associated with this norm. Since the Jacobian of the transformation $T_{\lambda}$ is $\lambda$ we see that $B_{\lambda}=T_{\lambda} B_{1}$ has measure $c \lambda$, where $c$ is an absolute constant.

Suppose that $M$ is an operator given (formally) by $g \rightarrow M g=(m \hat{g})^{\nu}$, where the multiplier $m$ is bounded and satisfies

$$
\begin{aligned}
m\left(T_{\lambda} y\right)=m(y), \quad\left(\Delta_{h}^{2} m\right)(y) & \equiv m(y+h)-2 m(y)+m(y-h) \\
& =O\left[\left(\frac{\|h\|}{\|y\|}\right)^{2 / 3}\right]
\end{aligned}
$$

for $\lambda>0$ and $y, h \in \mathbf{R}^{2}$. We claim that $M$ is a bounded operator on $H^{1}$ as well as on $H^{p}$ if $p$ is close to but less than 1. (Using the fact that $|h|^{2} \leqslant c\|h\|^{2 / 3}$ for $|h| \leqslant 1$ we see that condition (2.18) is satisfied by the two multipliers 
$i y_{2} /\left(y_{1}^{2}+i y_{2}\right)$ and $y_{1}^{2} /\left(y_{1}^{2}+i y_{2}\right)$ we are considering here.) This claim follows from

Lemma (2.19). Suppose $a$ is a 1-atom supported in the sphere $B_{1}$, then $M a=(m \hat{a})$ is a molecule centered at 0 . More precisely, $\int M a=0$ and

$$
\left(\int|(M a)(x)|^{2} d x\right)\left(\int|(M a)(x)|^{2}\|x\|^{1+\eta} d x\right)^{1 / \eta} \leqslant c_{\eta},
$$

where $0<\eta<1 / 3$ and $c_{\eta}$ is independent of $a$.

Once this lemma is established we see from Theorem $\mathrm{C}$ that $M a$ belongs to $H^{1}$. The fact that the operator $M$ commutes with the translations of $\mathbf{R}^{2}$ and the dilations $T_{\lambda}$ shows that it is well defined and is bounded on $H^{1}$.

In order to establish (2.19) we shall need the following result:

Lemma (2.20). Suppose $f \in L^{2}\left(\mathbf{R}^{2}\right)$ satisfies

$$
\int_{\mathbf{R}^{2}}|\hat{f}(x+h)-2 \hat{f}(x)+\hat{f}(x-h)|^{2} d x=\int_{\mathbf{R}^{2}}\left|\left(\Delta_{h}^{2} \hat{f}\right)(x)\right|^{2} d x \leqslant C\|h\|^{2 \alpha}
$$

for $2 \alpha>1$ then $\int_{\mathbf{R}^{2}}|f(x)|^{2}\|x\|^{\beta} d x<\infty$ when $0<\beta<2 \alpha$.

Proof. Suppose $R>1$ and $h=T_{1 / R} h^{\prime}$ where $\left|h^{\prime}\right|=\sqrt{\left(h_{1}^{\prime}\right)^{2}+\left(h_{2}^{\prime}\right)^{2}}=1$. If $R / 2<\|x\| \leqslant R$ then $1 / 2<\left\|T_{1 / R} x\right\| \leqslant 1$. It follows that $\left|e^{i \theta}-2+e^{-i \theta}\right|$ $\geqslant C_{0} \theta^{2}$ for an appropriate constant $C_{0}$, where $\theta=\left(T_{1 / R} h^{\prime}\right) \cdot x=h \cdot x$ $=\left(T_{1 / R} x\right) \cdot h^{\prime}$. Thus, by Plancherel's theorem and the assumption made in this lemma

$$
\begin{aligned}
C_{0} \int_{R / 2<\|x\| \leqslant R}|x \cdot h|^{4}|f(x)|^{2} d x & \leqslant \int_{R / 2<\|x\| \leqslant R}\left|e^{i x \cdot h}-2+e^{-i x \cdot h}\right|^{2}|f(x)|^{2} d x \\
& \leqslant C\|h\|^{2 \alpha}=C R^{-2 \alpha}\left\|h^{\prime}\right\| .
\end{aligned}
$$

We can now integrate both sides of this inequality with respect to $h^{\prime}$ over the unit circle in $\mathbf{R}^{2}$. Keeping in mind that $x \cdot h=\left(T_{1 / R} x\right) \cdot h^{\prime}$ is (essentially) the inner product of two unit vectors we obtain

$$
\int_{R / 2<\|x\| \leqslant R}|f(x)|^{2} d x \leqslant \text { (const) } R^{-2 \alpha} .
$$

Thus,

$$
\begin{aligned}
\int_{1<\|x\|}|f(x)|^{2}\|x\|^{\beta} d x & \leqslant \sum_{1}^{\infty} 2^{k \beta} \int_{2^{k-1}<\|x\| \leqslant 2^{k}}|f(x)|^{2} d x \\
& \leqslant \text { (const) } \sum_{1}^{\infty} 2^{k(\beta-2 \alpha)}<\infty .
\end{aligned}
$$

Since $\int_{\|x\| \leqslant 1}|f(x)|^{2}\|x\|^{\beta} d x$ is finite (since $f \in L^{2}\left(\mathbf{R}^{2}\right)$ ) the lemma is established.

Lemma (2.19) is a consequence of the result we have just obtained if we can establish that $\hat{f}=m \hat{a}$ satisfies the hypothesis of Lemma (2.20) with $\alpha=2 / 3$. In fact, 


$$
\begin{aligned}
\left(\Delta_{h}^{2} m \hat{a}\right)(y)= & m(y+h)\left(\Delta_{h}^{2} \hat{a}\right)(y)+\hat{a}(y)\left(\Delta_{h}^{2} m\right)(y) \\
& +[m(y+h)-m(y-h)][\hat{a}(y)-\hat{a}(y-h)] .
\end{aligned}
$$

To estimate the first term we use the fact that $a$ has support in $B_{1}$ and $m$ is bounded. Thus,

$$
\begin{aligned}
\int\left|m(y+h)\left(\Delta_{h}^{2} \hat{a}\right)(y)\right|^{2} d y & \leqslant\|m\|_{\infty} \int\left|e^{i x \cdot h}-2+e^{-i x \cdot h}\right|^{2}|a(x)|^{2} d x \\
& \leqslant(\text { const })|h|^{4} \leqslant(\text { const })\|h\|^{4 / 3}
\end{aligned}
$$

when $|h| \leqslant 1$ (this is clearly the only case we need to consider since $\|a\|_{2} \leqslant 1$ ).

In order to estimate the second term we first observe that $|\hat{a}(y)| \leqslant$ (const) $\cdot \min \{1,|y|\}$. Thus,

$$
\begin{aligned}
& \text { (const) } \int|\hat{a}(y)|^{2}\left|\left(\Delta_{h}^{2} m\right)(y)\right|^{2} d y \\
& \leqslant\|h\|^{4 / 3}\left\{\int_{\|y\| \leqslant 1} \frac{|y|^{2}}{\|y\|^{4 / 3}} d y+\int_{\|y\|>1}\|y\|^{-4 / 3} d y\right\} \\
& \leqslant(\text { const })\|h\|^{4 / 3} .
\end{aligned}
$$

Finally, in order to estimate the last term we observe that the assumption on the second difference $\Delta_{h}^{2} m$ in (2.18) implies

$$
|m(y+h)-m(y)|=O\left[(\|h\| /\|y\|)^{1 / 3}\right] .
$$

This last estimate follows from the argument preceding (3.5) on p. 44 of [64] applied to each variable separately. Secondly, we observe that

$$
|\hat{a}(y+h)-\hat{a}(y)|=O(|h|) \text { and } \int|\hat{a}(y+h)-\hat{a}(y)|^{2} d y=O\left(|h|^{2}\right) .
$$

Thus,

$$
\begin{aligned}
\int \mid m(y+h)- & \left.m(y-h)\right|^{2}|\hat{a}(y)-\hat{a}(y-h)|^{2} d y \\
& \leqslant C\|h\|^{2 / 3}\left(\int_{\|y\|<1} \frac{|h|^{2}}{\|y\|^{2 / 3}} d y+\int_{\|y\| \geqslant 1}|\hat{a}(y)-\hat{a}(y-h)|^{2} d y\right) .
\end{aligned}
$$

Using, again, the fact that $|h|$ is dominated by a constant times $\|h\|^{1 / 3}$ when $|h|$ $\leqslant 1$, we see that the last expression is $O\left(\|h\|^{4 / 3}\right.$ ) when $\|h\| \leqslant 1$. Lemma (2.19) is thus established.

Recall the question we raised originally when we introduced the heat equation: given $g \in L^{p}\left(\mathbf{R}^{2}\right)$ can we find a solution $u$ such that $\partial u / \partial x_{2}$ and $\partial^{2} u / \partial x_{1}^{2}$ belong to $L^{p}\left(\mathbf{R}^{2}\right)$ ? We then proceeded formally and exhibited a solution by means of equality (2.16). It is not clear, however, that such a solution is a function belonging to some natural function space associated with $L^{p}\left(\mathbf{R}^{2}\right)$, nor is it clear that the multiplier operators we considered act naturally on $L^{p}\left(\mathbf{R}^{2}\right)$. Another useful feature of the atomic 
theory of $H^{p}$ spaces is that if $g \in H^{1}\left(H^{1}=H^{1}\left(\mathbf{R}^{2},\|\|\right)\right.$ being defined with respect to the quasi-distance induced by \|\|$)$ then the solution

$$
u=\left[\left(1 /\left(y_{1}^{2}+i y_{2}\right)\right) \hat{g}\left(y_{1}, y_{2}\right)\right]^{\circ}
$$

belongs to $L^{3}\left(\mathbf{R}^{2}\right)$. This is easily seen in the following way: Suppose $a$ is an atom supported in $B_{1}$. Then from the estimates $|\hat{a}(x)| \leqslant($ const $) \min \{1,|x|\}$ $\leqslant$ (const) $\min \left\{1,\|x\|^{1 / 3}\right\}$ we obtain

$$
\text { (const) } \int \frac{|\hat{a}(x)|}{\|x\|} d x \leqslant \int_{\|x\| \leqslant 1}\|x\|^{-2 / 3} d x+\|a\|_{2}\left(\int_{\|x\|>1}\|x\|^{-2} d x\right)^{1 / 2} \leqslant C \text {, }
$$

where $C$ depends only on the dimension. Since

$$
\int\left|\hat{a}\left(T_{\lambda} x\right)\right| \frac{d x}{\|x\|}=\int|\hat{a}(x)| \frac{d x}{\|x\|} \leqslant C \text { for all } \lambda>0
$$

it follows that we have a nonisotropic version of Hardy's inequality

$$
\int_{\mathbf{R}^{2}} \frac{|\hat{g}(x)|}{\|x\|} d x<\infty
$$

whenever $g \in H^{1}\left(\mathbf{R}^{2},\|\|\right)$. Since $|\hat{a}(x)| \leqslant 1$ whenever $a$ is an atom in $H^{1}\left(\mathbf{R}^{2},\|\|\right)$ we must have, if $1 \leqslant p$,

$$
\int \frac{|\hat{a}(x)|^{p}}{\|x\|} d x \leqslant \int \frac{|\hat{a}(x)|}{\|x\|} d x \leqslant C .
$$

Thus, if $g=\sum \alpha_{i} a_{i} \in H^{1}\left(\mathbf{R}^{2},\|\|\right)$ then

$$
\left(\int_{\mathbf{R}^{2}}|\hat{g}(x)|^{p} \frac{d x}{\|x\|}\right)^{1 / p} \leqslant \Sigma\left|\alpha_{i}\right|\left(\int_{\mathbf{R}^{2}}\left|\hat{a}_{i}(x)\right|^{p} \frac{d x}{\|x\|}\right)^{1 / p} \leqslant C \sum\left|\alpha_{i}\right| .
$$

Consequently,

$$
\left(\int_{\mathbf{R}^{2}}|\hat{g}(x)|^{p} \frac{d x}{\|x\|}\right)^{1 / p} \leqslant C\|g\|_{H^{1}}
$$

whenever $g \in H^{1}\left(\mathbf{R}^{2},\|\|\right)$.

Equation (2.16) gives us a solution $u$ satisfying

$$
|\hat{u}(y)|=\left|\frac{\hat{g}(y)}{y_{1}^{2}+i y_{2}}\right| \leqslant \text { (const) } \frac{|\hat{g}(y)|}{\|y\|^{2 / 3}} .
$$

Applying (2.23) with $p=3 / 2$ we see that $\hat{u} \in L^{3 / 2}\left(\mathbf{R}^{2}\right)$. It now follows from the Hausdorff-Young theorem that $u \in L^{3}\left(\mathbf{R}^{2}\right)$. Furthermore, we have shown that the derivatives $\partial u / \partial x_{2}$ and $\partial^{2} u / \partial x_{1}^{2}$ (which certainly exist in the sense of distributions) belong to $H^{1}$. The fact that the operator defined by (2.21) maps $H^{1}$ into $L^{3}\left(\mathbf{R}^{2}\right)$ implies, by duality, that it maps $L^{3 / 2}\left(\mathbf{R}^{2}\right)$ into $B M O\left(\mathbf{R}^{2},\|\|\right)$. By applying an appropriate interpolation argument it can be shown that this operator maps $L^{p}$ into $L^{q}$, where $1 / p-1 / q=2 / 3$ and $1<p<3 / 2$. 
Singular integrals associated with the heat equation were studied first by Jones [39] and later by Fabes and Rivière [26]. Perhaps some of the results described here for $H^{p}$ spaces are new. Multipliers that are homogeneous relative to generalized dilations were studied by Rivière [49] and Krée [43].

In example (3) we considered other measures on $\mathbf{R}^{n}$, besides Lebesgue measure, which, together with the Euclidean distance, satisfied the basic inequality (2.1). Such measures arise naturally in several ways. For example radial functions in $L^{1}\left(\mathbf{R}^{n}\right)$ give rise to the study of the integrable functions on $(0, \infty)$ with respect to the measure $r^{n-1} d r$. The study of central functions on certain Lie groups also gives rise to measures $\omega(x) d x$ satisfying (2.1), where $\omega$ is an appropriate weight function. The case $\omega(x)=e^{b(x)}$, where $b$ is a BMO function, arises in factorization problems we shall discuss later. In all these cases we are dealing with weights belonging to the class $A^{\infty}$ of Muckenhoupt (see [13]) characterized by the inequality

$$
\frac{\omega(E \cap Q)}{\omega(Q)} \leqslant C\left(\frac{|E \cap Q|}{|Q|}\right)^{\delta},
$$

where $C$ and $\delta$ are constants depending only on $\omega,|E|$ denotes the Lebesgue measure of $E \subset \mathbf{R}^{n}, \omega(E)=\int_{E} \omega(x) d x$ and $Q$ is a cube with sides parallel to the axes.

It is an immediate consequence of $(2.24)$ that $\omega(\bar{Q}) \leqslant C \omega(Q)$ whenever $\bar{Q}$ is the cube concentric with $Q$ having sides twice the length of the latter (we shall use the letter $C$ to denote a constant, not always the same, that depends only on the dimension $n$ and the weight $\omega)$. Thus, $\left(\mathbf{R}^{n}, \omega\right)$, together with the Euclidean distance, is an example of a space of homogeneous type. Let $H^{1}\left(\mathbf{R}^{n}, \omega d x\right)$ denote the corresponding atomic Hardy space.

A basic general result relating these spaces to the more classical space $H^{1}\left(\mathbf{R}^{n}, d x\right)$ is the following:

Theorem (2.25). $H^{1}\left(\mathbf{R}^{n}, \omega d x\right)=\left\{f \omega^{-1}: f \in H^{1}\left(\mathbf{R}^{n}, d x\right)\right\}$.

In other words, the map $f \rightarrow f \omega$ is an isomorphism between the "weighted" Hardy space $H^{1}\left(\mathbf{R}^{n}, \omega d x\right)$ and the "ordinary" Hardy space $H^{1}\left(\mathbf{R}^{n}, d x\right)$.

In order to prove this theorem we make use of the following properties of weights satisfying the $A^{\infty}$ condition (2.24) (see [13]):

(a) there exists $\eta, C>0$ such that

$$
\left(\frac{1}{|Q|} \int_{Q} \omega^{1+\eta} d x\right)^{1 /(1+\eta)} \leqslant C \frac{\omega(Q)}{|Q|}
$$

(b) there exists $p>1$ and $C>0$ such that

$$
\frac{\omega(Q)}{|Q|}\left(\frac{1}{|Q|} \int_{Q} \omega^{-1 /(p-1)} d x\right)^{p-1} \leqslant C
$$

for all cubes $Q$. Suppose, now, that $a$ is a $(1, \infty)$-atom in $H^{1}\left(\mathbf{R}^{n}, \omega d x\right)$; that is, $a$ is supported in a cube $Q,|a(x)| \leqslant 1 / \omega(Q)$ and $\int a(x) \omega(x) d x=0$. We claim that $A=a \omega$ is (up to a multiplicative constant independent of $a$ ) a $(1,1+\eta$ )atom in $H^{1}\left(\mathbf{R}^{n}, d x\right)$. The fact that $A$ has mean zero is obvious; moreover, from (2.26) (a) we have 


$$
\left(\frac{1}{|Q|} \int|A(x)|^{1+\eta} d x\right)^{1 /(1+\eta)} \leqslant \frac{1}{\omega(Q)}\left(\int[\omega(x)]^{1+\eta} \frac{d x}{|Q|}\right)^{1 /(1+\eta)} \leqslant \frac{C}{|Q|} .
$$

Conversely, (2.26) (b) shows that if $A$ is a $(1, \infty)$-atom in $H^{1}\left(\mathbf{R}^{n}, d x\right)$ then $a=A / \omega$ is a $(1, p)$-atom in $H^{1}\left(\mathbf{R}^{n}, \omega d x\right)$. Theorem (2.25) now follows from Theorem A.

A consequence of (2.25) is the following characterization of radial functions belonging to $H^{1}\left(\mathbf{R}^{n}, d x\right)$ :

Corollary (2.27). Suppose $f$ is defined on $[0, \infty)$ then $x \rightarrow f(|x|)$ is a function in $H^{1}\left(\mathbf{R}^{n}, d x\right)$ if and only if $r \rightarrow f(|r|)|r|^{n-1}$ is a function in $H^{1}\left(\mathbf{R}^{1}, d r\right)$.

In order to show this result let us first assume that $f(|x|)=\sum \alpha_{i} a_{i}(x)$, where $a_{i}$ is a $(1, \infty)$-atom on $\mathbf{R}^{n}$ (not necessarily radial) and $\Sigma\left|\alpha_{i}\right|<\infty$. We claim that

$$
A_{i}(|r|)=\int_{|x|=1} a_{i}(|r| x) d \sigma(x),
$$

where $d \sigma(x)$ is the element of surface measure on the unit sphere in $\mathbf{R}^{n}$, is an atom in $H^{1}\left(\mathbf{R}^{1},|r|^{n-1} d r\right)$ times a constant that depends only on the dimension. If the support of $a=a_{i}$ contains the origin this is obviously true..$^{27}$ If the support of $a$ is at a distance $R>0$ from the origin and $d$ is the diameter of this support, then there are $N \geqslant C[R / d]^{n-1}$ rotations $\rho_{1}, \rho_{2}, \ldots, \rho_{N}$ such that $a\left(\rho_{j} x\right), j=1, \ldots, N$, have disjoint supports. Hence,

$$
A(|r|)=\frac{1}{N} \int_{|x|=1}\left(\sum_{j=1}^{N} a\left(r \rho_{j} x\right)\right) d \sigma(x)
$$

is bounded by $(1 / N)\left(1 / d^{n}\right) \leqslant C\left(1 / d R^{n-1}\right)$ (since the supports of the functions appearing in the sum are disjoint). But the last expression is dominated by a constant times

$$
1 /\left(\int_{R}^{R+d} r^{n-1} d r\right)
$$

In order to obtain the converse we start out with a $(1, \infty)$-atom $A$ in $H^{1}\left(\mathbf{R}^{1},|r|^{n-1} d r\right)$ and observe that if its support contains 0 then the radial function it defines is (essentially) an atom in $H^{1}\left(\mathbf{R}^{n}, d x\right)$. If the support of $A$ is the interval $(R, R+d), R>0$, we can "reverse" the construction we just gave in order to obtain an appropriate atomic decomposition in $\mathbf{R}^{n}$ of the radial function defined by $A$. More generally, many results concerning weighted $H^{p}$ spaces on $\mathbf{R}^{n}$ were developed in the thesis of one of our Ph.D. students, J. Garcia-Cuerva [32]. The class of weights considered here on $\mathbf{R}^{n}$ admits a useful straightforward extension to spaces of homogeneous type.

The notion of weighted $H^{1}$ spaces has interesting applications in the compact case as well. The following result, as we shall see, can be obtained by using this notion:

\footnotetext{
27 We remark, once and for all, that when we talk about the support of an atom we really mean the smallest sphere containing the support of an atom.
} 
THEOREM (2.28). Suppose $f \in L^{\mathrm{I}}(\mathbf{R})$ has mean 0 and is supported in the interval $[-1,1]$. Then $f=\sum \alpha_{j} a_{j}$, where $\sum\left|\alpha_{j}\right|<\infty$ and the $a_{j}$ 's are $(1, \infty)$ atoms supported in $[-1,1]$, if and only if $\int_{-1}^{1}|\tilde{f}(x)| d x<\infty$, where

$$
\tilde{f}(x)=\text { P. V. } \frac{1}{\pi} \int \frac{f(t)}{x-t} d t .
$$

Since the Hilbert transform $\tilde{a}$ of a $(1, \infty)$-atom satisfies $\int_{-\infty}^{\infty}|\tilde{a}(x)| d x=\|\tilde{a}\|_{1}$ $\leqslant C, C$ independent of $a$ (see the argument following (2.12)), we have

COROllary (2.29). If $f \in L^{1}(\mathbf{R})$ is supported in an interval I and has mean zero then $\tilde{f} \in L^{1}(I)$ implies $\tilde{f} \in L^{1}(\mathbf{R})$.

Using Theorem (2.28) and the same argument we gave to establish Theorem (2.25) we obtain a characterization of an $H^{1}$ space associated with the space of homogeneous type we introduced in example (5). In order to state this result let us write $\omega_{\alpha, \beta}(x)=(1-x)^{\alpha}(1+x)^{\beta},-1 \leqslant x \leqslant 1,-1<\alpha, \beta$,

$$
\left(H_{\alpha, \beta} f\right)(x)=\frac{1}{\omega_{\alpha, \beta}(x)} \text { P.V. } \int_{-1}^{1} \frac{f(t) \omega_{\alpha, \beta}(t)}{x-t} d t
$$

and let $H^{1}\left([-1,1], \omega_{\alpha, \beta}(t) d t\right)$ be the atomic Hardy space generated by the $(1, \infty)$-atoms defined in terms of the measure $\omega_{\alpha, \beta}(t) d t$. We then have

Corollary (2.30). $f \in H^{1}\left([-1,1], \omega_{\alpha, \beta}(t) d t\right)$ if and only if $f$ and $H_{\alpha, \beta}$ f belong to $L^{1}\left(\omega_{\alpha, \beta}(t) d t\right)$.

We now pass to the proof of (2.28). Let $f$ satisfy the hypotheses of this theorem; then, $f_{\mathrm{e}}(\theta)=f(\cos \theta)|\sin \theta|$ is a function in $L^{1}([-\pi, \pi], d \theta)$. If $x=\cos \psi$ then

$$
\begin{aligned}
\tilde{f}(x) & =\frac{1}{\sin \psi} \text { P.V. } \frac{1}{\pi} \int_{0}^{\pi} f_{\mathrm{e}}(\theta) \frac{\sin \psi}{\cos \psi-\cos \theta} d \theta \\
& \equiv-\frac{1}{2 \sin \psi} \text { P.V. } \frac{1}{\pi} \int_{-\pi}^{\pi} f_{\mathrm{e}}(\theta) \cot \frac{\psi-\theta}{2} d \theta
\end{aligned}
$$

But

$$
f_{\mathrm{e}}^{c}(\psi)=\frac{1}{\pi} \text { P.V. } \int_{-\pi}^{\pi} f_{\mathrm{e}}(\theta) \cot \frac{\psi-\theta}{2} d \theta
$$

is the conjugate function of $f_{\mathrm{e}}$. That is, $f_{\mathrm{e}}+i f_{\mathrm{e}}^{c}$ is the boundary value of the analytic function associated with $f_{0}$ we described at the beginning of $\$ 1$. Moreover, it follows from the change of variables we made above (and the fact that $f_{\mathrm{e}}^{c}$ is an odd function) that $\int_{-1}^{1}|\tilde{f}(x)| d x=C \int_{-\pi}^{\pi}\left|f_{\mathrm{e}}^{c}(\psi)\right| d \psi$. Consequently, $f_{\mathrm{e}}$ belongs to $H^{1}(T)\left(=\operatorname{Re} H^{1}(T)\right)$ and, therefore, has an atomic decomposition

$$
f_{\mathrm{e}}(\theta)=\sum \alpha_{j} a_{j}(\theta)
$$

where $\sum\left|\alpha_{j}\right|<\infty$ and $a_{j}$ is a $(1, \infty)$-atom of mean 0 supported in the interval $\boldsymbol{I}_{j}$. We now claim that it can be assumed that if $0 \leqslant \theta \leqslant \pi$ the sum in (2.31) 
involves only atoms $a_{j}$ whose support $I_{j}$ lies in $[0, \pi]$. To see this we first observe that since $f_{\mathrm{e}}$ is even

$$
f_{\mathrm{e}}(\theta)=\sum \alpha_{j} \frac{a_{j}(\theta)+a_{j}(-\theta)}{2} .
$$

If $0 \in I_{j}$ then $a_{j}^{\prime}(\theta)=\left(a_{j}(\theta)+a_{j}(-\theta)\right) / 2$ is supported in $I_{j}^{\prime}=\left\{\theta: \theta \in I_{j}\right.$ or $\left.-\theta \in I_{j}\right\}$. We also have $\int_{-\pi}^{\pi} a_{j}^{\prime}=0$ and, since $a_{j}^{\prime}$ is even, $\int_{0}^{\pi} a_{j}^{\prime}=0$. Moreover, $\left|a_{j}^{\prime}(\theta)\right| \leqslant 1 /\left|I_{j}\right| \leqslant 2 /\left|[0, \pi) \cap I_{j}^{\prime}\right|$. Thus, $a_{j}^{\prime} / 2$, restricted to $[0, \pi]$ is a $(1, \infty)$ atom. A similar argument applies to the case $\pi \in I_{j}$ (see footnote 8). If neither 0 nor $\pi$ belong to $I_{j}$ then either $a_{j}(\theta)$ or $a_{j}(-\theta)$ is 0 for $0 \leqslant \theta \leqslant \pi$ and there is nothing to prove.

Having made this restriction of the representation $(2.31)$ to $[0, \pi]$ we shall now show that $A_{j}$, defined on $[-1,1]$ by

$$
A_{j}(\cos \theta) \sin \theta=a_{j}(\theta)
$$

is a $(1, p)$-atom in the space $H^{1}([-1,1], d t)$ for certain values of $p>1$ (up to an inessential multiplicative constant). In fact, let $J_{j}$ be the image of $I_{j}$ under the map $\theta \rightarrow \cos \theta=t$. Then $A_{j}$ is supported by $J_{j},\left|J_{j}\right|=\int_{I_{j}} \sin \theta d \theta$ and $\int_{J_{j}} A_{j}(t) d t=\int_{I_{j}} a_{j}(\theta) d \theta=0$. Thus, we need only show that

$$
\left(\int_{J_{j}}\left|A_{j}(t)\right|^{p} \frac{d t}{\left|J_{j}\right|}\right)^{1 / p} \leqslant \frac{C}{\left|J_{j}\right|} .
$$

We can rewrite this inequality in the form:

$$
\left(\int_{I_{j}}\left|A_{j}(\cos \theta) \sin \theta\right|^{p} \frac{(\sin \theta)^{1-p}}{\left|J_{j}\right|} d \theta\right)^{1 / p} \leqslant \frac{C}{\left|J_{j}\right|} .
$$

Since $\left|A_{j}(\cos \theta) \sin \theta\right|=\left|a_{j}(\theta)\right| \leqslant 1 /\left|I_{j}\right|$ the desired inequality follows from the easily derived condition

$$
\left(\frac{1}{\left|I_{j}\right|} \int_{I_{j}} \sin \theta d \theta\right)^{1-(1 / p)}\left(\frac{1}{\left|I_{j}\right|} \int_{I_{j}}(\sin \theta)^{1-p} d \theta\right)^{1 / p} \leqslant C,
$$

for $1<p<2$. (In fact, this is the $A^{q}$ condition of Muckenhoupt [13] for the weight $\sin \theta$ valid for $q>2$, where $1 / p+1 / q=1$.) Theorem (2.28) now. follows from Theorem A.

It would be of interest to obtain higher dimensional extensions of Theorem (2.28) and Corollary (2.29).

Corollary (2.30) is relevant to the study of Jacobi polynomial expansions. These are polynomials, $P_{n}^{\alpha, \beta}$, of order $n$ that are eigenfunctions of the differential operator

$$
D_{\alpha, \beta}=\left(1-x^{2}\right) \frac{d^{2}}{d x^{2}}+(\beta-\alpha-(\alpha+\beta+2) x) \frac{d}{d x}
$$

corresponding to the eigenvalue $-n(n+\alpha+\beta+1), \alpha, \beta>-1$. The Jacobi functions, $Q_{n}^{\alpha, \beta}$, of the second kind are also eigenfunctions of this differential 
operator corresponding to the same eigenvalue; moreover (see p. 171 of [25]), they are related to the Jacobi polynomials by the formula

$$
Q_{n}^{\alpha, \beta}(x)=\left(H_{\alpha, \beta} P_{n}^{\alpha, \beta}\right)(x) .
$$

Thus, expansions in terms of Jacobi polynomials are transformed into expansions in terms of Jacobi functions of the second kind. Corollary (2.30) gives us some information about these expansions when the former represents $H^{1}\left([-1,1], \omega_{\alpha, \beta}(t) d t\right)$.

When $\alpha=\beta$ the $P_{n}^{\alpha, \alpha}$ are the ultraspherical polynomials. Muckenhoupt and Stein [47] have developed a theory of $H^{p}$ spaces involving expansions in terms of these polynomials. When $\alpha=(n-2) / 2, n$ an integer, it is well known that these expansions arise from the study of spherical harmonics. Using this geometrical interpretation and the techniques used to establish Theorem (2.25) one can see that the space $H^{1}\left([-1,1], \omega_{\alpha, \alpha}(t) d t\right)$ introduced here and the $H^{1}$ space studied by Muckenhoupt and Stein are identical when $\alpha=(n-2) / 2$. It would be of interest to see what is the relation between these two spaces for other indices $\alpha$. The analogous problem involving Hankel transforms has been studied by J. Garcia-Cuerva [32].

An important topic involving expansions in terms of Jacobi polynomials is the study of the multiplier operators associated with these expansions. These operators can be defined in a manner completely analogous to the way they are defined in the theory of Fourier series. We recall that the polynomials $P_{n}^{\alpha, \beta}$ can be obtained by the Gram-Schmidt orthonormalization process from the monomials (relative to the natural inner product defined by the weight $\omega_{\alpha, \beta}$ ). Thus, if $f \in L^{2}\left([-1,1], \omega_{\alpha, \beta}(x) d x\right)$ then

$$
f=\sum_{n=0}^{\infty} \hat{f}_{n} P_{n}^{(\alpha, \beta)},
$$

where $\hat{f}_{n}=\int_{-1}^{1} f(x) P_{n}^{\alpha, \beta}(x) \omega_{\alpha, \beta}(x) d x$. Hence, if $\left\{m_{n}\right\}$ is a bounded sequence the operator $M$ mapping $f$ into the function having expansion $\sum_{n=0}^{\infty} m_{n} \hat{f}_{n} P_{n}^{\alpha, \beta}$ is well defined and maps into $L^{2}\left([-1,1], \omega_{\alpha, \beta}(x) d x\right)$. These are the operators commuting with the differential operator $D_{\alpha, \beta}$. As is the case in the study of Fourier series and integrals it is natural to inquire what properties of $f$ are preserved under the action of $M$. Since $[-1,1]$, together with the measure induced by the weight $\omega_{\alpha, \beta}$, is a space of homogeneous type, the theory of singular integrals connected with these spaces can be applied to obtain sufficient conditions under which $M$ is a bounded operator on

$$
L^{p}\left([-1,1], \omega_{\alpha, \beta}(x) d x\right), \quad 1<p<\infty
$$

(see [17] and [20]). A careful reading of these results and the methods used will show that these conditions also imply the boundedness of $M$ on the corresponding atomic $H^{p}$ spaces when $p(\leqslant 1)$ is close to 1 (compare with the discussion preceding and following conditions (2.14) and (2.15)). Because of Corollary B it then follows that $M$ preserves the "natural" Lipschitz condition associated with this space of homogeneous type: 


$$
|f(x)-f(y)| \leqslant C\left|\int_{x}^{y}(1-t)^{\alpha}(1+t)^{\beta} d t\right|^{1 / p-1}
$$

Specifically, one can establish the following extension of a multiplier theorem of Connett and Schwartz [20]:

THeOREM (2.32). Suppose $\alpha \geqslant \beta>-1 / 2$ and $k$ is the largest integer not exceeding $\alpha+2$, then if $\left\{m_{n}\right\}$ is a bounded sequence satisfying

$$
\sum_{R \leqslant n<2 R}\left|\Delta^{k} m_{n}\right|^{2} \leqslant c R^{1-2 k},
$$

where $\Delta^{k}$ is the kth difference operator, the multiplier operator $M$ associated with $\left\{m_{n}\right\}$ is bounded on $L^{p}\left([-1,1], \omega_{\alpha, \beta}(x) d x\right)$ for $1<p<\infty$ and on $H^{1}\left([-1,1], \omega_{\alpha, \beta}(x) d x\right)$.

Let us now pass to the situation encountered when $X=[0,1), \mu$ is Lebesgue measure and the quasi-distance is given in terms of dyadic intervals as described in example (4). We shall refer to this situation as "the dyadic case". Many aspects of harmonic analysis become particularly simple and elegant in this dyadic case. In particular, the atomic $H^{1}$ space can easily be seen to coincide with the space of all $L^{1}$ functions whose Hardy-Littlewood maximal function $f^{*}$ is integrable. That is, if we let $f^{*}(x)=\sup _{I}(1 /|I|)\left|\int_{I} f\right|$, where the supremum is taken over all dyadic intervals $I$ containing $x$, then $f$ is in atomic $H^{1}$ if and only if $f^{*} \in L^{1} .^{28}$

In order to establish this equivalence we first observe that it is easy to see that $\left\|a^{*}\right\|_{1} \leqslant 1$ whenever $a$ is a $(1, \infty)$-atom. In fact, if $I$ and $J$ are two nondisjoint dyadic intervals in $[0,1)$ then either $I \subset J$ or $I \supset J$. From this it follows immediately that if $a$ is supported in $I$ then $a^{*}$ is also supported in $I$ and satisfies $a^{*}(x) \leqslant 1 /(|I|)$; therefore, $\left\|a^{*}\right\|_{1} \leqslant 1$ and we see that

$$
\left\|f^{*}\right\|_{1} \leqslant\|f\|_{H^{1}}
$$

whenever $f$ is in atomic $H^{1}$. Conversely, if $f$ and $f^{*}$ are in $L^{1}$ we shall show that $f$ has an atomic decomposition $\sum \alpha_{j} a_{j}$ with $\sum\left|\alpha_{j}\right| \leqslant 8\left\|f^{*}\right\|_{1}$. Without loss of generality we can assume $\int_{0}^{1} f=0$. We shall now obtain the desired representation of $f$ by means of the Calderón-Zygmund decomposition of $f$. We first consider $U_{k}^{\prime}=\left\{x \in[0,1): f^{*}(x)>2^{k}\right\}$ for $k$ an integer and we let $U_{k}^{\prime}$ $=\cup_{i} I_{k}^{i}$ where the $I_{k}^{i}$ s $\mathrm{s}$ are maximal dyadic open subintervals of $U_{k}^{\prime}$ (and, thus, for $k$ fixed, they are disjoint). Now, each $I_{k}^{i}$ is contained in a (unique) dyadic interval, $J_{k}^{i}$, of twice its length. Since each $I_{k}^{i}$ is maximal, $J_{k}^{i}$ contains a point outside $U_{k}^{\prime}$ and, thus, $m_{J_{k}}(f)=\left(1 /\left|J_{k}^{i}\right|\right) \int_{J_{k}^{i}} f$ has absolute value less than or equal to $2^{k}$. Let $U_{k}=\cup_{i} J_{k}^{i}$; we may assume that $J_{k}^{i}$, for $k$ fixed, are mutally disjoint (recall that two intersecting dyadic intervals have the property that one must contain the other). The Calderón-Zygmund decomposition is then, $f=g_{k}+b_{k}$, where $g_{k}=\left(1-\chi_{U_{k}}\right) f+\sum_{i} m_{J_{k}^{i}}(f) \chi_{J_{k}}$. Clearly, $\left|g_{k}\right| \leqslant 2^{k}$ and the $b_{k}$ 's are supported in $U_{k}$ and satisfy $\int_{J^{k}} b_{k}=0$. As $k \rightarrow \infty$ we have $g_{k} \rightarrow f$ (trivially in the a.e. sense and, also, in the $L^{1}$-sense since the convergence is

${ }^{28} f^{*}$ is the natural analog of the nontangential maximal operator $P^{*}$ we introduced in the beginning of $\$ 1$ (see [2]). 
dominated by $\left.2 f^{*}\right)$. Also, $\lim _{k \rightarrow-\infty}\left\|g_{k}\right\|_{1}=0$. Thus, $f=\sum_{-\infty}^{\infty}\left(g_{k+1}-g_{k}\right)$ $=\sum_{-\infty}^{\infty}\left(b_{k}-b_{k+1}\right)$. Moreover, $\left|b_{k}-b_{k+1}\right|=\left|g_{k+1}-g_{k}\right| \leqslant 2^{k+1}$. Since $U_{k+1} \subset U_{k}$ and each interval $J_{k+1}^{j}$ must be a subinterval of some $J_{k}^{j}$ we have $\int\left(b_{k}-b_{k+1}\right) \chi_{J_{k}^{i}}=0$. Therefore, $a_{k}^{i}=\left(1 / 2^{k+1}\left|J_{k}^{i}\right|\right)\left[b_{k}-b_{k+1}\right] \chi_{J_{k}^{i}}$ is a $(1, \infty)$ atom and $f=\sum_{k=-\infty}^{\infty} 2^{k+1} \sum_{i}\left|J_{k}^{i}\right| a_{k}^{i}$. Furthermore,

$$
\begin{aligned}
\left\|f^{*}\right\|_{1} & =\int_{0}^{\infty}\left|\left\{x:[0,1): f^{*}(x)>\lambda\right\}\right| d \lambda \geqslant \sum_{k=-\infty}^{\infty}\left|U_{k+1}^{\prime}\right| 2^{k} \\
& \geqslant \sum_{k=-\infty}^{\infty}\left|U_{k}\right| 2^{k} 2^{-2}=\sum_{k=-\infty}^{\infty} 2^{k+1} 2^{-3} \sum_{i}\left|J_{k}^{i}\right| .
\end{aligned}
$$

But this shows that $\|f\|_{H^{1}} \leqslant 8\left\|f^{*}\right\|_{1}$ and the claim is established.

Let us now pass to some illustrations of how these methods apply to the study of harmonic analysis on homogeneous spaces (example (7)). We begin with an extension of Hardy's inequality associated with the surface $\Sigma_{n-1}$ of the unit sphere in $\mathbf{R}^{n}, n \geqslant 3$. Let $t^{\prime}, x^{\prime}, y^{\prime}, \ldots$ denote points of $\Sigma_{n-1}, d t^{\prime}, d x^{\prime}, d y^{\prime}$, $\cdots$ the elements of Lebesgue surface measure on $\Sigma_{n-1}$ (which is normalized so that $\Sigma_{n-1}$ has measure 1). $H_{k}$ denotes the space of homogeneous harmonic polynomials of degree $k$ restricted to $\Sigma_{n-1}$ and $Z_{x^{\prime}}^{(k)}$ the zonal harmonic of degree $k$ with pole $x^{\prime}$. The defining property of $Z_{x^{\prime}}^{(k)}$ is that it is the (unique) element of $H_{k}$ such that

$$
Y\left(x^{\prime}\right)=\int_{\Sigma_{n-1}} Y\left(t^{\prime}\right) Z_{x^{\prime}}^{(k)}\left(t^{\prime}\right) d t^{\prime}
$$

whenever $Y \in H_{k} \cdot Z_{x^{\prime}}^{(k)}$ is also determined (up to a multiplicative constant) by the property that it is a member of $H_{k}$ that is invariant under the action of all rotations $\rho$ of $\mathbf{R}^{n}$ that leave $x^{\prime}$ fixed (see pp. 146-147 of [57]). From this and the fact that $Z_{x^{\prime}}^{(k)}\left(x^{\prime}\right)=d_{k}=$ dimension of $H_{k}$ we obtain the following simple expression for zonal harmonics:

$$
Z_{x^{\prime}}^{(k)}\left(t^{\prime}\right)=d_{k} \int_{\Sigma_{n-2}}\left[t^{\prime} \cdot\left(i y^{\prime}+x^{\prime}\right)\right]^{k} d y^{\prime},
$$

where $\Sigma_{n-2}$ is being identified with all those vectors $y^{\prime} \in \Sigma_{n-1}$ orthogonal to $x^{\prime}$. From this we see that

$$
\left|\frac{\partial^{2}}{\partial t_{i} \partial t_{j}} Z_{x^{\prime}}^{(k)}\left(t^{\prime}\right)\right| \leqslant 2 d_{k} k(k-1) .
$$

Now let us consider

$$
Z_{x^{\prime}}^{(k)}\left(t^{\prime}\right)-Z_{x^{\prime}}^{(k)}\left(x^{\prime}\right)=\left(\nabla Z_{x^{\prime}}^{(k)}\right)\left(x^{\prime}\right) \cdot\left(t^{\prime}-x^{\prime}\right)+E^{(k)}\left(x^{\prime}, t^{\prime}\right) .
$$

The error term involves the second derivatives of $Z_{x^{\prime}}^{(k)}$ (evaluated at some point on the segment joining $x^{\prime}$ and $\left.t^{\prime}\right)$ and products of the type $\left(x_{l}^{\prime}-t_{l}^{\prime}\right)$ $\cdot\left(x_{j}^{\prime}-t_{j}^{\prime}\right)_{2}$ Because of $(2.34)$, therefore, we have $\left|E^{(k)}\left(x^{\prime}, t^{\prime}\right)\right| \leqslant c d_{k} k^{2}$ - $\left|x^{\prime}-t^{\prime}\right|^{2}$. Moreover, it follows from the integral representation of $Z_{x^{\prime}}^{(k)}$ that

$$
\left(\nabla Z_{x^{\prime}}^{(k)}\right)\left(x^{\prime}\right)=k d_{k} x^{\prime}
$$


Consequently, $\nabla Z_{x^{\prime}}^{(k)}\left(x^{\prime}\right) \cdot\left(t^{\prime}-x^{\prime}\right)=k d_{k}\left(x^{\prime} \cdot t^{\prime}-1\right)=-\left(k d_{k} / 2\right)\left|x^{\prime}-t^{\prime}\right|^{2}$. It follows that

$$
\left|Z_{x^{\prime}}^{(k)}\left(t^{\prime}\right)-d_{k}\right|=\left|Z_{x^{\prime}}^{(k)}\left(t^{\prime}\right)-Z_{x^{\prime}}^{(k)}\left(x^{\prime}\right)\right| \leqslant c d_{k} k^{2}\left|x^{\prime}-t^{\prime}\right|^{2} .
$$

If $f \in L^{1}\left(\Sigma_{n-1}\right)$ its projection onto $H_{k}$ is

$$
f_{k}\left(x^{\prime}\right)=\int_{\Sigma_{n-1}} f\left(t^{\prime}\right) Z_{x^{\prime}}^{(k)}\left(t^{\prime}\right) d t^{\prime}
$$

(this follows from the defining property of the zonal harmonics). We thus have the orthogonal development of $f$ in terms of spherical harmonics (see [57, Chapter 4, §2]): $f \sim \sum f_{k}$. We shall now derive the following extension of Hardy's inequality:

THEOREM (2.36). If $f \in H^{1}\left(\Sigma_{n-1}\right)$ then

$$
\sum_{k=1}^{\infty} \frac{\left\|f_{k}\right\|_{2}}{k^{n / 2}}=\sum_{k=1}^{\infty} k^{-n / 2}\left(\int_{\Sigma_{n-1}}\left|f_{k}\left(x^{\prime}\right)\right|^{2} d x^{\prime}\right)^{1 / 2} \leqslant c\|f\|_{H^{\prime}}
$$

In order to establish $(2.36)$ we choose a $(1, \infty)$-atom supported in a "sphere" of radius $R$ about $x^{\prime}$. Then, by the "mean 0 " property of $a$ :

$$
a_{k}\left(y^{\prime}\right)=\int_{\Sigma_{n-1}} a\left(t^{\prime}\right) Z_{y^{\prime}}^{(k)}\left(t^{\prime}\right) d t^{\prime}=\int_{\Sigma_{n-1}} a\left(t^{\prime}\right)\left[Z_{y^{\prime}}^{(k)}\left(t^{\prime}\right)-Z_{y^{\prime}}^{(k)}\left(x^{\prime}\right)\right] d t^{\prime} .
$$

Thus, by Minkowski’s integral inequality

$$
\left\|a_{k}\right\|_{2} \leqslant \int_{\Sigma_{n-1}}\left|a\left(t^{\prime}\right)\right|\left\{\int_{\Sigma_{n-1}}\left|Z_{y^{\prime}}^{(k)}\left(t^{\prime}\right)-Z_{y^{\prime}}^{(k)}\left(x^{\prime}\right)\right|^{2} d y^{\prime}\right\}^{1 / 2} d t^{\prime} .
$$

Since $Z_{v^{\prime}}^{(k)}\left(u^{\prime}\right)=Z_{u^{\prime}}^{(k)}\left(v^{\prime}\right)$ (see p. 143 of [57]) we have

$$
\begin{aligned}
& \int_{\Sigma_{n-1}}\left|Z_{y^{\prime}}^{(k)}\left(t^{\prime}\right)-Z_{y^{\prime}}^{(k)}\left(x^{\prime}\right)\right|^{2} d y^{\prime} \\
& =\int_{\Sigma_{n-1}}\left\{\left[Z_{t^{\prime}}^{(k)}\left(y^{\prime}\right)\right]^{2}+\left[Z_{x^{\prime}}^{(k)}\left(y^{\prime}\right)\right]^{2}-2 Z_{t^{\prime}}^{(k)}\left(y^{\prime}\right) Z_{x^{\prime}}^{(k)}\left(y^{\prime}\right)\right\} d y^{\prime} \\
& =Z_{t^{\prime}}^{(k)}\left(t^{\prime}\right)+Z_{x^{\prime}}^{(k)}\left(x^{\prime}\right)-2 Z_{x^{\prime}}^{(k)}\left(t^{\prime}\right)=2\left[d_{k}-Z_{x^{\prime}}^{(k)}\left(t^{\prime}\right)\right] .
\end{aligned}
$$

Hence, we can use (2.35) to obtain the estimate

$$
\left\|a_{k}\right\|_{2} \leqslant c \int_{\Sigma_{n-1}}\left|a\left(t^{\prime}\right)\right| k \sqrt{d_{k}}\left|x^{\prime}-t^{\prime}\right| d t^{\prime} .
$$

Since $d_{k} \leqslant c k^{n-2}$ (see [57, p. 145]) and $\left|x^{\prime}-t^{\prime}\right| \leqslant R$ for $t^{\prime}$ in the support of $a$ we have

$$
\left\|a_{k}\right\|_{2} \leqslant c R k \cdot k^{(n-2) / 2}=c R k^{n / 2} .
$$

Making use of this inequality and the obvious estimate for the $L^{2}$ norm of an atom we finally obtain 


$$
\begin{aligned}
\sum_{k=1}^{\infty} \frac{\left\|a_{k}\right\|_{2}}{k^{n / 2}} & =\sum_{k \leqslant 1 / R} \frac{\left\|a_{k}\right\|_{2}}{k^{n / 2}}+\sum_{k>1 / R} \frac{\left\|a_{k}\right\|_{2}}{k^{n / 2}} \\
& \leqslant \sum_{k \leqslant 1 / R} c R+\left(\sum_{k=1}^{\infty}\left\|a_{k}\right\|_{2}^{2}\right)^{1 / 2}\left(\sum_{k>1 / R} k^{-n}\right)^{1 / 2} \\
& \leqslant c^{\prime}\left(1+\|a\|_{2} R^{(n-1) / 2}\right) \leqslant c^{\prime \prime} .
\end{aligned}
$$

Theorem (2.36) is an immediate consequence of this inequality.

The version of Hardy's inequality we have just discussed assumes a special form on the sphere $\Sigma_{3}$. This sphere can be identified in a natural way with the group $S U(2)$ of unitary $2 \times 2$ matrices with determinant 1 . This group is the simplest noncommutative semisimple compact Lie group to analyze and is particularly useful for obtaining insight into problems in harmonic analysis associated with more general Lie groups. We shall need to introduce some notation in order to illustrate some of these features.

We write $z=\left(z_{1}, z_{2}\right)=\left(x_{1}+i x_{2}, x_{3}+i x_{4}\right)\left(\in \mathbf{C}^{2}\right)$ for $x=\left(x_{1}, x_{2}, x_{3}, x_{4}\right)$ $\in \mathbf{R}^{4}$. To $x$ (or $z$ ) we can associate the $2 \times 2$ matrix

$$
u=u(x)=-i\left(\begin{array}{cc}
-\bar{z}_{2} & z_{1} \\
\bar{z}_{1} & z_{2}
\end{array}\right) .
$$

Clearly, det $u=\left|z_{1}\right|^{2}+\left|z_{2}\right|^{2}=x_{1}^{2}+x_{2}^{2}+x_{3}^{2}+x_{4}^{2}$ and, hence, $\Sigma_{3}$ corresponds to $S U(2)$ and normalized Lebesgue measure on $\Sigma_{3}$ corresponds to Haar measure on $S U(2)$. The irreducible representations of $S U(2)$ can be realized explicitly on the spaces $\mathcal{P}^{l}$ of homogeneous polynomials of degree $2 l, l=0,1 / 2,1,3 / 2,2, \ldots$, in $\left(\omega_{1}, \omega_{2}\right)=\omega \in \mathbf{C}^{2}$. That is, $\mathscr{P}^{l}$ consists of all the polynomials of the form $p(\omega)=\sum_{j=-l}^{l} a_{j} \omega_{1}^{l-j} \omega_{2}^{l+j}$ and the mapping of $u \in S U(2)$ into the transformation $T_{u}^{l}$ defined by

$$
\left(T_{u}^{l} p\right)(\omega)=p\left(u^{\prime} \omega\right)
$$

where $u^{\prime}$ is the transpose of $u$, is a representation. Moreover, $T_{u}^{l}$ is unitary with respect to an appropriate inner product for which

$$
p_{j}(\omega)=\frac{1}{\sqrt{(l-j) !} \sqrt{(l+j) !}} \omega_{1}^{l-j} \omega_{2}^{l+j},
$$

$j=-l,-l+1, \ldots, l$, form an orthonormal basis (see [17] for further details). The matrix entries of $T^{l}$ relative to this basis are usually denoted by $t_{k, j}^{l}(u),-l \leqslant k, j \leqslant l$, and are defined by the relation

$$
\begin{aligned}
(-i)^{2 l} & \frac{\left(-\bar{z}_{2} \omega_{1}+\bar{z}_{1} \omega_{2}\right)^{l-j}\left(z_{1} \omega_{1}+z_{2} \omega_{2}\right)^{l+j}}{\sqrt{(l-j) !(l+j) !}} \\
& =\sum_{k=-l}^{l} t_{k, j}^{l}(u(z)) \frac{\omega_{1}^{l-k} \omega_{2}^{l+k}}{\sqrt{(l-k) !(l+k) !}} .
\end{aligned}
$$

It follows that $t_{k, j}^{l}(u(z))=t_{k, j}^{l}(u(x))$ are homogeneous polynomials in $x$ of degree $2 l$. Moreover, their restrictions to $\Sigma_{3}$ form an orthogonal basis for the 
space $H^{2 l}$ of spherical harmonics of degree $2 l$. The expansion of functions on $\Sigma_{3}$ in terms of spherical harmonics turns out to be equivalent to the PeterWeyl expansion of functions on $S U(2)$. The latter can be expressed in the following way: if $f \in L^{2}(S U(2))$ then

$$
f(u)=\sum_{2 l=0}^{\infty}(2 l+1) \operatorname{tr}\left(\hat{f}(l) T_{u}^{l}\right)
$$

where

$$
\hat{f}(l)=\int_{S U(2)} f(u) T_{u^{-1}}^{l} d u,
$$

and the convergence of this series to $f(u)$ is valid in the $L^{2}$-norm. Moreover,

$$
\int|f(u)|^{2} d u=\sum_{2 l=0}^{\infty}(2 l+1) \mid\|\hat{f}(l)\|^{2},
$$

where $\|\hat{f}(l)\|||$ denotes the Hilbert-Schmidt norm of $\hat{f}(l)$. It follows from these considerations and, in particular, (2.37), that the projection, $f_{2 l}$, of $f$ onto $H^{2 l}$ satisfies $\left\|f_{2 l}\right\|_{2}^{2}=(2 l+1)\|\hat{f}(l)\|^{2}$. Thus, Hardy's inequality (2.36) when $n$ $=4$ is equivalent to

$$
\sum_{2 l=0}^{\infty} \frac{\|\hat{f}(l)\|}{(2 l+1)^{3 / 2}}<c\|f\|_{H^{1}}
$$

whenever $f \in H^{1}(S U(2))$. By using the interpolation Theorem D and the two inequalities (2.37) and (2.38) we obtain the following generalization of Paley's inequality:

$$
\sum_{2 l=0}^{\infty}(2 l+1)^{(5 p / 2)-4}\|\hat{f}(l)\| \leqslant c_{p}\|f\|_{p}
$$

whenever $f \in L^{p}(S U(2)), 1<p \leqslant 2$.

As is the case in $\mathbf{R}^{n}$ and $T$, the study of the behavior of multiplier operators is enhanced by appropriate use of properties of $H^{p}$ spaces. We first remark that bounded operators $M$ on $L^{2}(S U(2))$ that commute with left translations correspond to matrix multiplier operators; that is, if $f \in L^{2}(S U(2))$ then $M f$ has the Peter-Weyl expansion

$$
(M f)(u)=\sum_{2 l=0}^{\infty}(2 l+1) \operatorname{trace}\left[\hat{M}(l) \hat{f}(l) T_{u}^{l}\right],
$$

where $\hat{M}(l)$ is a $(2 l+1) \times(2 l+1)$ matrix whose operator norm $\|\hat{M}(l)\|$ does not exceed a constant $C$ independently of $l$. It turns out that the space $H^{1}\left(\Sigma_{3}\right)=H^{1}(S U(2))$, defined in terms of atoms associated with the usual Euclidean distance on $\Sigma_{3},{ }^{29}$ can be characterized by certain "Riesz" transforms that correspond to the following matrix multiplier operators:

29 On $S U(2)$ this distance is more naturally written in terms of the Hilbert-Schmidt norm: $d(u, v)=\|u-v\| \|$ when $u, v \in S U(2)$. 


$$
\left[\hat{M}_{ \pm}(l)\right]_{k, j}=\frac{\sqrt{(l \pm j)(l \mp j+1)}}{2 l+1} \delta_{j \mp 1, k}, \quad\left[\hat{H}_{ \pm}(l)\right]_{k, j}=\frac{l \pm k}{2 l+1} \delta_{k, j},
$$

where $\delta_{k, j}$ is the Kronecker delta. These operators are related to certain generalized Cauchy-Riemann equations in $\mathbf{R}^{4}$ in a manner similar to the way the conjugate function operator on the circle $T$ is related to the CauchyRiemann equations in $\mathbf{R}^{2}$ (again, see [17] for further details and motivation).

The use of the atomic Hardy spaces in the study of multiplier operators is somewhat complicated by the fact that the multipliers consist of sequences of matrices. As in the previous cases, one can characterize the matricial Fourier transform of a molecule centered at the identity. In order to show that a multiplier preserves $H^{1}$ it is enough to show that it maps atoms into molecules (this follows from Theorem C). Let us discuss this situation in greater detail. Let us write $\rho(u)=\|u-1\|^{3}$ where 1 is the identity element of $S U(2)$. Then a molecule centered at $\mathbf{1}$ is a function $\alpha(\mathrm{u})$ satisfying

(2.40) $\int \alpha(u) d u=0$ and $\left(\int|\alpha(u)|^{2} d u\right)\left(\int|\alpha(u)|^{2}[\rho(u)]^{1+\varepsilon}\right)^{1 / \varepsilon} \leqslant 1$.

If we let $\varepsilon=1 / 3$ we obtain a weighted Plancherel theorem (see p. 114 of [17]) which, applied to $\alpha$, takes the form

$$
\int_{S U(2)}|\alpha(u)|^{2}\|u-\mathbf{1}\|\left\|^{4} d u=\sum_{2 l=0}^{\infty}(2 l+1)\right\| \Delta^{2} \hat{\alpha}(l) \|^{2},
$$

where

$$
\Delta^{2} \hat{\alpha}(l)=\int \alpha(u)\|u-\mathbf{1}\|^{2} T_{u^{-1}}^{l} d u
$$

is a certain second order "difference" operator defined on sequences of matrices $\hat{\alpha}(l)$. It can be shown that

$$
\left[\Delta^{2} \hat{\alpha}(l)\right]_{k, j}=2[\hat{\alpha}(l)]_{k, j}-\sum_{\gamma, \delta= \pm 1 / 2} A_{k}^{l}(\gamma, \delta) A_{j}^{l}(\gamma, \delta)[\hat{\alpha}(l+\gamma)]_{k+\delta, j+\delta}
$$

where

$$
A_{k}^{l}(\gamma, \delta)=\sqrt{\frac{1}{2}+\frac{\gamma(1+4 \delta k)}{2 l+1}} .
$$

Thus, with $\varepsilon=1 / 3(2.40)$ can be expressed by

$$
\begin{aligned}
& \hat{\alpha}(0)=0 \text { and } \\
& \left(\left.\sum_{2 l=0}^{\infty}(2 l+1)\|\hat{\alpha}(l)\|\right|^{2}\right)\left(\sum_{2 l=0}^{\infty}(2 l+1)\left\|\Delta^{2} \hat{\alpha}(l)\right\|^{2}\right)^{1 / 3} \leqslant 1 .
\end{aligned}
$$

The direct method for checking whether the matrices $\hat{\alpha}(l)=\hat{M}(l) \hat{a}(l)$ are the coefficients of a molecule when $a$ is an atom centered at 0 can be used here. This procedure, however, is technically complicated since it involves estimates on $\Delta^{2}(\hat{M}(l) \hat{a}(l))$. Fortunately, it can be shown that, if the inequality 


$$
\sum_{2 l=0}^{\infty}(2 l+1)\left\|\mid \Delta^{2}(\hat{M}(l) \hat{a}(l))\right\|^{2} \leqslant c \sum_{2 l=0}^{\infty}(2 l+1)\left\|\Delta^{2} \hat{a}(l)\right\|^{2}
$$

holds for some special central atoms $a$, then $M$ maps $H^{1}$ boundedly into itself (a careful reading of the proof of Theorem (3.1) on p. 81 of [17] shows that $M$ is a convolution operator with a kernel satisfying condition (2.15)). Conditions on $\hat{M}(l)$ that insure the validity of the last inequality can be stated in a relatively simple manner; we thus obtain the following result:

THEOREM (2.42). Let $\{\hat{M}(l)\}$ be a sequence of $(2 l+1) \times(2 l+1)$ matrices satisfying $\left\|\Delta^{j} \hat{M}(l)\right\| \leqslant c l^{-j+1 / 2}, j=0,1,2$, where

$$
\Delta^{0} \hat{M}(l)=\hat{M}(l) \text { and } \Delta^{1} \hat{M}(l)=\sum_{\varepsilon, \delta= \pm 1 / 2} \varepsilon A_{k}^{l}(\varepsilon, \delta) A_{j}^{l}(\varepsilon, \delta)[\hat{M}(l+\varepsilon)]_{k+\delta, j+\delta} \text {, }
$$

then $M$ is a bounded multiplier operator on $L^{p}(S U(2)), 1<p<\infty$, and on $H^{p}$ for $p$ sufficiently close to, but not exceeding, 1.

Two remarks are in order: (a) the conditions on $\Delta^{j} \hat{M}(l)$ are quite easy to check for the Riesz transforms $M_{ \pm}$and $H_{ \pm}$(see pp. 134 and 135 of [17]); (b) If the matrices $\hat{M}(l)$ are diagonal, Theorem (2.42) assumes a more famillar form: Let $\mu_{m}^{l},-l \leqslant m \leqslant l$, be the entries of the diagonal matrix $\hat{M}(l)$; if $\mu_{m}^{l}$ and $l^{2}\left(2 \mu_{m}^{l}-\mu_{m+\delta}^{l+1 / 2}-\mu_{m-\delta}^{l-1 / 2}\right)$ are $O(1)$ for $\delta=-1 / 2,1 / 2$ then $M$ is bounded on $L^{p}(S U(2))$ for $1<p<\infty$ and on $H^{1}$.

The analysis of the Fourier transform described here for $S U(2)$ can, in principle, be extended to other classical compact groups. The main difficulties involve the choice of a special basis in each representation space and the calculation of certain Clebsch-Gordan coefficients that lead to formulae for operators corresponding to $\Delta^{1}$ and $\Delta^{2}$. The spaces $H^{p}$ for compact semisimple Lie groups were introduced in [16] using generalized Cauchy-Riemann equations. The basic subharmonicity result obtained there permits one to establish the duality of $H^{1}$ and $B M O$ by the methods of Fefferman and Stein. This duality result, in turn, shows that the $H^{1}$ defined in terms of the CauchyRiemann equations is the atomic $H^{1}$ space.

We now discuss example (9) and its connection with the theory of analytic functions of several complex variables. If

$$
B_{n}=\left\{z \in \mathbf{C}^{n}:|z|^{2}=\left|z_{1}\right|^{2}+\left|z_{2}\right|^{2}+\cdots+\left|z_{n}\right|^{2}<1\right\}
$$

then $\partial B_{n}=\Sigma_{2 n-1}$.

A function $F(z)$ holomorphic in $B_{n}$ is said to belong to $\mathcal{H C}^{p}\left(\partial B_{n}\right)$ if

$$
\|F\|_{\mathscr{S}_{\mathcal{C}}}^{p}=\sup _{r<1} \int_{\left|z^{\prime}\right|=1}\left|F\left(r z^{\prime}\right)\right|^{p} d z^{\prime}<\infty,
$$

where $d z^{\prime}$ is normalized Lebesgue measure on $\Sigma_{2 n-1}$. The space $\mathcal{K}^{2}$ is of particular interest. It can be identified with the closed subspace of $L^{2}\left(\partial B_{n}\right)$ consisting of boundary functions of holomorphic functions.

The projection operator $P$ from $L^{2}\left(\partial B_{n}\right)$ onto $\mathcal{H}^{2}$ is given in terms of the Szegö kernel $(1-z \cdot \bar{\omega})^{-n}$. We first define the transformation $S$ : 


$$
(S f)(z)=\int_{\partial B_{n}} \frac{f\left(\omega^{\prime}\right)}{\left(1-z \cdot \bar{\omega}^{\prime}\right)^{n}} d \omega^{\prime} \text { for }|z|<1 .
$$

We now observe that $d(z, \omega)=|1-z \cdot \bar{\omega}|^{1 / 2}$ is a distance on $\partial B_{n}$ which is particularly well adapted to the study of this kernel. In fact, $m(z, \omega)$ $=|1-z \cdot \bar{\omega}|^{n}$ is a quasi-distance equivalent to the measure of the smallest $d$ sphere containing $z$ and $\omega,|z|=|\omega|=1$, and the basic inequality (2.14) is verified for the Szegö kernel: for $|z|=|\omega|=\left|\omega_{0}\right|=1$

$$
\left|\frac{1}{(1-z \cdot \bar{\omega})^{n}}-\frac{1}{\left(1-z \cdot \overline{\omega_{0}}\right)^{n}}\right| \leqslant\left[\frac{m\left(\omega, \omega_{0}\right)}{m\left(z, \omega_{0}\right)}\right]^{1 / 2 n} \frac{C_{1}}{m\left(z, \omega_{0}\right)}
$$

if $m\left(z, \omega_{0}\right)>C_{2} m\left(\omega, \omega_{0}\right)$.

It can also be shown (see [42]) that for $z \in \partial B_{n}$, and $f \in L^{2}\left(\partial B_{n}\right)$

$$
(P f)(z)=\lim _{r \rightarrow 1}(S f)(r z)=\frac{1}{2} f(z)+\lim _{\varepsilon \rightarrow 0} \int_{m(z, \omega)>\varepsilon} \frac{f(\omega)}{(1-z \cdot \bar{\omega})^{n}} d \omega .
$$

It now follows from the general theory developed previously that the projection operator can be defined as a bounded operator on $L^{p}, 1<p<\infty$, and $H^{p}, 1-\eta<p \leqslant 1$, and it preserves the Lipschitz character of $f$ (with respect to $d$ ). Using the methods of Fefferman and Stein [29] it can be shown that the dual of $\mathcal{H}^{1}\left(\partial B_{n}\right)$ is $P(B M O)$ (see [14]). This result is equivalent to:

TheOREM (2.44). Let $F \in \mathcal{H}^{1}\left(\partial B_{n}\right)$. Then there exist atoms $a_{j}$ such that

$$
F(z)=\sum \lambda_{j}\left(P a_{j}\right)(z) \equiv \sum \lambda_{j} A_{j}(z)
$$

with

$$
\sum\left|\lambda_{j}\right| \leqslant c\|F\|_{\mathcal{H}^{1}}
$$

thus, $P\left(H^{1}\right)=\mathcal{H}^{1}$.

The functions $A_{j}(z)=\left(P a_{j}\right)(z)$, called holomorphic atoms, are easily seen to be molecules. A related fact (see [14]) is that any holomorphic atom $A$ can be written in the form

$$
A(z)=\sum_{i=1}^{N} B_{i}(z) C_{i}(z)
$$

with $B_{i}, C_{i} \in \mathcal{F}^{2}$ satisfying $\sum_{i=1}^{N}\left\|B_{i}\right\|_{\mathcal{C}^{2}}\left\|C_{i}\right\|_{\mathcal{C}^{2}} \leqslant c$, where $N$ and $c$ depend only on the dimension $n$. From this follows the factorization result:

THEOREM (2.46). Let $F \in \mathcal{H}^{1}\left(\partial B_{n}\right)$. Then there are functions $G_{j}, H_{j} \in \mathcal{H}^{2}$ such that $F=\sum_{j} G_{j} H_{j}$ and

$$
\sum_{j}\left\|G_{j}\right\|_{\mathcal{S C}^{2}}\left\|H_{j}\right\|_{\mathcal{C}^{2}} \leqslant c\|F\|_{\mathcal{C l}^{1}} \text {. }
$$

Let us indicate briefly how (2.45) is proved. We may assume without loss of generality that an atom $a$ is supported in a sphere $S$ centered at 1 
$=(0,0, \ldots, i)$ (the "North pole"). We let $\varphi_{S}=(1 /|S|) \chi_{S}$ and write

$$
1-\bar{\omega} \cdot z=-\bar{\omega}_{0} \cdot z_{0}+\left(1+i z_{n}\right)\left(i \bar{\omega}_{n}\right)+\left(1-i \bar{\omega}_{n}\right)
$$

for $z=\left(z_{1}, z_{2}, \ldots, z_{n}\right), \omega=\left(\omega_{1}, \omega_{2}, \ldots, \omega_{n}\right) \in \partial B_{n}$, where $z_{0}=\left(z_{1}, z_{2}, \ldots\right.$, $\left.z_{n-1}, 0\right)$ and $\omega_{0}=\left(\omega_{1}, \omega_{2}, \ldots, \omega_{n-1}, 0\right)$. We have

$$
(1-\bar{\omega} \cdot z)^{n}=\sum_{|J|+k+l=n} d_{J, k, l} \bar{\omega}_{0}^{J}\left(1-i \bar{\omega}_{n}\right)^{k} \bar{\omega}_{n}^{l} z_{0}^{J}\left(1+i z_{n}\right)^{l},
$$

where $J=\left(j_{1}, \ldots, j_{n-1}\right),|J|=j_{1}+j_{2}+\cdots+j_{n-1}$ and $z_{0}^{J}=z_{1}^{j_{1}} z_{2}^{j_{2}} \cdots z_{n-1}^{j_{n-1}}$. We write

$$
1=\int_{\partial B_{n}} \varphi_{S}(\omega) d \omega=\sum_{|J|+k+l=n} z_{0}^{J}\left(1+i z_{n}\right)^{l} D_{J, k, l}(z),
$$

where

$$
D_{J, k, l}(z)=d_{J, k, l} \int_{\partial B_{n}} \frac{\bar{\omega}_{0}^{J}\left(1-i \bar{\omega}_{n}\right)^{k} \bar{\omega}_{n}^{l} \varphi_{S}(\omega)}{(1-z \cdot \bar{\omega})^{n}} d \omega
$$

Thus,

$$
A(z)=\sum_{|J|+k+l=n} z_{0}^{J}\left(1+i z_{n}\right)^{l} D_{J, k, l}(z) A(z) .
$$

We now claim that each summand can be split into a product of the form $B(z) C(z)$ with $\|B\|_{\mathcal{F C}^{2}}\|C\|_{\mathcal{F}^{2}} \leqslant C$. This is achieved by using inequality (2.43) in order to estimate $A(z)$ and by making rough estimates for $D_{J, k, l}(z)$ when $z$ is far from $S$ (see [14] for details).

Other spaces $\mathcal{F}^{p}$ of holomorphic functions for which this theory can be used are the spaces of functions $F$ that are holomorphic in $B_{n}$ and satisfy

$$
\int_{|z|<1}|F(z)|^{p}\left(1-|z|^{2}\right)^{m} d \nu(z)<\infty,
$$

where $d \nu(z)$ is the element of Lebesgue measure on $\mathbf{C}^{n}$. The case $m=0$ is of particular interest. Here the projection, $P$, of $L^{2}\left(B_{n}\right)$ onto $\mathcal{T}^{2}\left(B_{n}\right)$ is given in terms of the Bergman kernel:

$$
(P f)(z)=\frac{n !}{\pi^{n}} \int_{B_{n}} \frac{f(\omega)}{(1-z \cdot \bar{\omega})^{n+1}} d v(\omega) .
$$

Unlike the preceding case, this projection is not a singular integral transform; however, $P$ is unbounded on $L^{1}\left(B_{n}\right)$. If we introduce the quasi-distance $d$ of example (10), $B_{n}$ becomes a space of homogeneous type and $P$ maps (atomic) $H^{1}$ into itself. As was the case for $\partial B_{n}, P H^{1}=\mathcal{H}^{1}\left(B_{n}\right)$ and factorizations analogous to the ones in Theorem (2.46) are obtainable.

In this connection we point out that when $n=1$ the dual space of $\mathcal{H}^{1}\left(B_{1}\right)$ can be represented by the class $\Lambda_{*}$ of smooth functions of Zygmund (see [14]). The fact that $P H^{1}=\mathscr{H}^{1}\left(B_{1}\right)$ allows us also to represent this dual space by holomorphic BMO functions on the solid disc $B_{1}$. From this it follows easily 
that smooth functions are characterized by the property that the derivatives of their analytic extensions to the interior of the disc belongs to the class $B M O\left(B_{1}\right)$. It would be of interest to extend this relation to higher dimensions.

The methods we have just described extend to more general strictly pseudoconvex domains (see [56]). It would be interesting to obtain similar factorization results in this more general setting. Another important related study involves the polydisc and its distinguished boundary $T^{n}$. In this situation the possibility of obtaining corresponding results is completely open.

We have considered $\Sigma_{2 n-1}=\partial B_{n}$ as a point set that furnished us two different examples of a space of homogeneous type. We did this by first employing the ordinary Euclidean metric and, then, the nonisotropic distance $d(z, \omega)=|1-z \cdot \bar{\omega}|^{1 / 2}$ (in both cases Lebesgue measure was used). It is natural to compare the Hardy spaces that arise in these two situations and one does find basic differences. At this point, however, we would like to point out that when dealing with holomorphic functions many results are easily reduced to the case of the 1-dimensional torus (where $n=1$ ). Let us illustrate this point by considering a version of Hardy's inequality associated with $\mathcal{H}^{1}\left(\partial B_{n}\right)$.

Let $F(z)=\sum_{0}^{\infty} F_{k}(z)$ be holomorphic in $B_{n}$, where $F_{k}(z)=\sum_{|\alpha|=k} c_{\alpha} z^{\alpha}$ is the projection of $F$ onto the polynomials in $z=\left(z_{1}, z_{2}, \ldots, z_{n}\right)$ that are homogeneous of degree $k$. Let $g_{z}\left(e^{i \theta}\right)=F\left(e^{i \theta} z\right)=\sum_{0}^{\infty} F_{k}(z) e^{i k \theta}$. By $(1.10)$ we then have

$$
\sum_{1}^{\infty} \frac{\left|F_{k}(z)\right|}{k} \leqslant \int_{-\pi}^{\pi}\left|g_{z}\left(e^{i \theta}\right)\right| d \theta .
$$

Integrating $z \in \partial B_{n}$ (we now assume $F \in \mathcal{H}^{1}\left(\partial B_{n}\right)$ ) we obtain

$$
\sum_{1}^{\infty} \frac{\left\|F_{k}\right\|_{1}}{k} \leqslant c\|F\|_{1} \text {. }
$$

Since $\left\|F_{k}\right\|_{2} \leqslant k^{(n-1) / 2}\left\|F_{k}\right\|_{1},(2.47)$ implies the following result involving the Taylor coefficients of $F$ :

$$
\sum_{k=1}^{\infty} \frac{1}{\sqrt{k^{n+1}(n+k-1) !}}\left(\sum_{|\alpha|=k} \alpha_{1} ! \alpha_{2} ! \cdots \alpha_{n} !\left|c_{\alpha}\right|^{2}\right)^{1 / 2} \leqslant c\|F\|_{1} .
$$

Other results on $\mathcal{F}^{p}\left(\partial B_{n}\right)$ that are reducible to the 1-dimensional case by similar arguments can be found in [51].

Consider a function $B(z)$ on $B_{n}$ for which $b_{z}\left(e^{i \theta}\right)=B\left(e^{i \theta} z\right)$ satisfies

$$
\sup _{z}\left\|b_{z}\left(e^{i \theta}\right)\right\|_{B M O\left(\partial B_{1}\right)}=c<\infty .
$$

From the previous argument we then obtain

$$
\left|\int_{\partial B_{n}} F \bar{B} d \sigma\right|=\lim _{r \rightarrow 1}\left|\int_{\partial B_{n}} F(r z) \overline{B(r z)} d \sigma(z)\right| \leqslant c \int_{\partial B_{n}}|F| d \sigma .
$$

Consequently, $B \in B M O\left(\partial B_{n}\right)$. There are, however, functions $B$ in $B M O\left(\partial B_{n}\right)$ for which $\sup _{z}\left\|b_{z}\left(e^{i \theta}\right)\right\|_{B M O\left(\partial B_{1}\right)}=\infty$. For example, $B\left(z_{1}, z_{2}\right)$ $=\sum_{0}^{\infty} z_{1}^{2 k}$ furnishes us an example when $n=2$. For such a function the last 
inequality cannot be obtained by a reduction to the 1-dimensional case.

In these last few examples the spaces of homogeneous type involved were compact. There exists a noncompact space that is closely connected to $B_{n}$ and $\partial B_{n}$. Let us give some details about this situation when $n=2$. In this case we shall consider the generalized half-plane

$$
D=D_{2}=\left\{\left(z_{1}, z_{2}\right) \in \mathbf{C}^{2}: \operatorname{Im} z_{1}>\left|z_{2}\right|^{2}\right\}
$$

which is the image of $B_{2}$ under the Caley transform

$$
Z=\left(z_{1}, z_{2}\right)=i\left(\frac{1-\omega_{1}}{1+\omega_{1}}, \frac{\omega_{2}}{1+\omega_{1}}\right) \text {. }
$$

This mapping of $\left(\omega_{1}, \omega_{2}\right)$ onto $\left(z_{1}, z_{2}\right)$ extends to the boundary $\partial B_{2}$ which is mapped onto $\partial D=\left\{\left(z_{1}, z_{2}\right): \operatorname{Im} z_{1}=\left|z_{2}\right|^{2}\right\}=\left\{\left(t+i|z|^{2}, z\right): t \in \mathbf{R}, z\right.$ $\in \mathbf{C}\}$. There is a group $G$ that acts in a simply transitive fashion on $\partial D$. The elements of $G$ can be identified with the set $\mathbf{R} \times \mathbf{C}$; the image of $\left(t+i|z|^{2}, z\right)$ $\in \partial D$ under the action on $(\tau, \xi) \in G$ is

$$
\left(t+\tau+2 \operatorname{Im} z \bar{\xi}+i|z+\xi|^{2}, z+\xi\right) .
$$

If we write $(\tau, \xi)=\left(x_{3},\left(x_{1}+i x_{2}\right) / 2\right)$ and make the correspondence of $(\tau, \xi)$ with the matrix

$$
\left(\begin{array}{ccc}
1 & x_{1} & x_{3}+\left(x_{1} x_{2} / 2\right) \\
0 & 1 & x_{2} \\
0 & 0 & 1
\end{array}\right)
$$

(see example (11)) we obtain an isomorphism between the group of transformations $G$ and the group $H$ of such matrices (under matrix multiplication). The latter group is an example of a Heisenberg group. This gives us a natural identification of the spaces $\partial D, G$, and $H$ in each of which we can introduce ordinary Lebesgue measure; it is easy to check that the latter is a Haar measure for $G$ and $H$.

The Cauchy-Szegö kernel associated with $D$ has the form

$$
k(Z, W)=k\left(\left(z_{1}, z_{2}\right),\left(\omega_{1}, \omega_{2}\right)\right)=c\left(i\left(\bar{\omega}_{1}-z_{1}\right)-2 z_{2} \bar{\omega}_{2}\right)^{-2} .
$$

Using this kernel we obtain the projection operator of $L^{2}(\partial D)$ onto the subspace consisting of boundary values of holomorphic functions (the method is the same as that used in the study of $\left.B_{n}\right)$. Observe that if $Z=\left(z_{1}, z_{2}\right)$ $=\left(t+i|z|^{2}, z\right) \in \partial D$ then $k(Z, 0)=c\left(|z|^{2}-i t\right)^{-2}$. We see, thus, that the "measure norm" $m(Z)=\left(t^{2}+|z|^{4}\right)$ is particularly well adapted to the study of this kernel. Moreover, $m(Z)$ is homogeneous with respect to the dilations $\delta_{\lambda}, \lambda>0$, given by $\delta_{\lambda} Z=\left(\lambda^{1 / 2} t+i\left|\lambda^{1 / 4} z\right|^{2}, \lambda^{1 / 4} z\right)$. We obtain a space of homogeneous type whose point set is $\partial D$ (or $G$, or $H$ ). The kernel $k(Z, W)$ satisfies inequality (2.14) and, thus, preserves the associated space $H^{1}$ (or $H^{p}$ for $p$ near but less than 1$)$.

These $H^{p}$ spaces are also useful for the study of partial differential operators commuting with the dilations $\delta_{\lambda}$ (these generalize homogeneous differential 
operators). An interesting example, studied by Folland and Stein [31], is the operator

$$
\mathfrak{L}_{\alpha}=-\frac{\partial^{2}}{\partial \bar{z} \partial z}-|z|^{2} \frac{\partial^{2}}{\partial t^{2}}+i \frac{\partial}{\partial t}\left(z \frac{\partial}{\partial z}-\bar{z} \frac{\partial}{\partial \bar{t}}\right)+i \alpha \frac{\partial}{\partial t},
$$

when $\alpha$ is not an odd integer. The fundamental solutions for $\varrho_{\alpha}$ turn out to be

$$
c_{\alpha}\left(\frac{|z|^{2}+i t}{|z|^{2}-i t}\right)^{\alpha / 2} \frac{1}{\left(|z|^{4}+t^{2}\right)^{1 / 2}} .
$$

Folland and Stein were able to use this, together with the theory of singular integrals, in order to obtain sharp estimates for solutions of the equation $\mathfrak{L}_{\alpha} f=g$. Singular integrals, $H^{p}$ spaces and their maximal characterizations for the Heisenberg group are also treated by D. Geller in his forthcoming thesis at Princeton University under the direction of E. M. Stein. The theory of Hardy spaces developed here, and its uses in the study of singular integrals, can also be used for this purpose and provides corresponding estimates in $H^{p}$ and $B M O$.

The Heisenberg group $H$ we have considered is an example of a class of Lie groups considered by Rothschild and Stein [50]: the free nilpotent Lie groups of step $r$. Briefly, these groups arise in the following important construction. Let $M$ be a manifold and $X_{1}, \ldots, X_{n}$ vector fields which, together with their commutators up to order $r$, span the tangent space at each point of $M$. Let us assume that these vector fields are free up to step $r$; that is, at each point of $M$ the dimension of the space spanned by $\left\{X_{1}, \ldots, X_{n}\right\}$ and their commutators up to order $r$ is equal to the dimension of the free nilpotent Lie algebra of step $r$. As was shown by Rothschild and Stein, this is not a handicap in the study of the general situation. Then, there exists, at each point of $M$, a local coordinate system with respect to which the $X_{j}$ 's are (essentially) the generators of a free nilpotent Lie algebra. The homogeneous norm function on the Lie algebra can be used in order to define a quasi-distance on $M$ that is well adapted for the study of differential operators involving the vector fields $X_{j}$. (In the above example, $M=\partial D$, the $X_{j}$ 's can be chosen as a real basis for the holomorphic vector fields on $\partial D$.) This program is carried out in general in [50] for generalizations of the operators $\mathcal{L}_{\alpha}$. A priori estimates are then obtained by studying singular integrals, first on the corresponding group and then on the space of homogeneous type $M$. It would be of interest to characterize the spaces $H^{1}(M)$ in terms of these singular integrals.

Perhaps the simplest example of a noncompact group is the group $\mathbf{Z}$ (or $\mathbf{Z}^{n}$ ) of integers. This group, with the usual distance and counting measure, is a space of homogeneous type. The spaces $H^{p}$ are easily characterizable in terms of the discrete Hilbert transform:

$$
\left\{f_{k}\right\} \in H^{1} \text { if and only if } \Sigma\left|f_{k}\right|<\infty \text { and } \Sigma\left|\tilde{f}_{k}\right|<\infty,
$$

where $\tilde{f}_{k}=\sum_{j \neq k} f_{j} /(k-j)$.

Various characterizations of the Fourier transform 


$$
\hat{f}(\theta)=\sum f_{k} e^{i k \theta}
$$

can be given as before.

This example shows that the differentiable structure of the group is not strictly necessary in order to obtain a singular integral characterization of the space $H^{1}$. A striking illustration of this fact occurs in the $p$-adic case (see example (12)). There, it turns out that there exists a system of singular integral operators, analogous to the Riesz transforms, which characterize $H^{1}$. (See [61] where it is also shown that some of the subharmonicity inequalities of $\mathbf{R}^{n}$ have an appropriate analogue.)

3. Proofs of the principal results. In order to establish Theorem A we shall need to know some general facts about the geometry and analysis of spaces of homogeneous type $X$. Many of these facts are proved in [17]; when such a result is not contained in this monograph we shall give a proof of it here. Throughout this section $A$ denotes the constant in the inequality (2.1) and $K$ is the constant occurring in the "triangle inequality" satisfied by the quasidistance $d$.

Theorem (3.1) (VItAli-WIener TYPe Covering lemma). Suppose $E$ is a bounded set (i.e. contained in a sphere) in $X$ such that for each $x \in E$ there exists $r(x)>0\left(\right.$ thus, $\left\{S_{r(x)}(x)\right\}$ is a covering of $\left.E\right)$, then there exists a sequence of points $x_{j} \in E$ such that $\left\{S_{r\left(x_{j}\right)}\left(x_{j}\right)\right\}$ is a disjoint family of spheres while $\left\{S_{4 K r}\left(x_{j}\right)\left(x_{j}\right)\right\}$ is a covering of $E$.

(See Theorem (1.2) on p. 69 of [17].)

TheOREM (3.2) (WhitNey TYPe COVering Lemma). Suppose $U \subseteq X$ is an open bounded set and $C \geqslant 1$. Then there exists a sequence of spheres $\left\{S_{j}\right\}$ $=\left\{S_{s_{i}}\left(x_{j}\right)\right\}$ satisfying

(i) $U=\cup_{j} S_{j}$;

(ii) there exists a constant $M=M(A, C, K)$ such that no point of $X$ belongs to more than $M$ of the spheres $\bar{S}_{j} \equiv S_{C_{s_{j}}}\left(x_{j}\right)$;

(iii) $\overline{\bar{S}}_{j} \cap(X-U) \neq \varnothing$ for each $j$, where $\overline{\bar{S}_{j}}=S_{3 K C S_{j}}\left(x_{j}\right)$.

Proof. For each $x, r(x)=\left(1 / 8 C K^{2}\right) d\left(x, U^{\prime}\right)=\left(1 / 8 C K^{2}\right) \min \{d(x, y): y$ $\notin U\}>0$ since $U$ is open and $X-U=U^{\prime} \neq \varnothing$. By (3.1) we can find a mutally disjoint sequence of spheres $\left\{S_{r\left(x_{j}\right)}\left(x_{j}\right)\right\}$ such that $U$ is covered by the spheres $S_{4 K r\left(x_{j}\right)}\left(x_{j}\right)$. On the other hand, if $y \in S_{4 K C r\left(x_{j}\right)}\left(x_{j}\right)$ then

$$
d\left(x_{j}, y\right)<4 K C r\left(x_{j}\right)=(1 / 2 K) d\left(x_{j}, U^{\prime}\right)<d\left(x_{j}, U^{\prime}\right) .
$$

It follows that if we put $s_{j}=4 K r\left(x_{j}\right)$ we have $S_{s_{j}}\left(x_{j}\right)=S_{j} \subset S_{C s_{j}}\left(x_{j}\right)=\bar{S}_{j}$ $\subset U$. Thus,

$$
U=\cup S_{j} \leqq U \bar{S}_{j} \subset U .
$$

In particular, (i) is established. Moreover, since $3 K C s_{j}=12 K^{2} \operatorname{Cr}\left(x_{j}\right)$ $=(3 / 2) \rho\left(x_{j}, U^{\prime}\right)$ we must have $\overline{\bar{S}}_{j} \cap U^{\prime} \neq \varnothing$ and (iii) is established.

In order to show that (ii) is valid we shall make use of the following geometric property of spaces of homogeneous type (see Lemma (1.1) on p. 68 of [17]): 
(3.4) There exists a constant $N=N(A, K)$ such that $S_{r}(x)$ cannot contain more than $N^{n}$ points $\left\{x_{i}\right\}$ satisfying $d\left(x_{i}, x_{j}\right)>r 2^{-n}$ when $i \neq j(n=1,2$, $3, \ldots)$.

Now suppose $x \in \bar{S}_{j}$. We claim that $C s_{j}<d\left(x, U^{\prime}\right) \equiv R$ since

$$
2 C K s_{j}=d\left(x_{j}, U^{\prime}\right) \leqslant K\left[d\left(x_{j}, x\right)+d\left(x, U^{\prime}\right)\right]<K\left[C s_{j}+d\left(x, U^{\prime}\right)\right] .
$$

Consequently, if $y \in \bar{S}_{j}, d(x, y) \leqslant K\left[d\left(x, x_{j}\right)+d\left(x_{j}, y\right)\right]<2 K R$ and we must have $\bar{S}_{j} \subset S_{2 K R}(x)$. Moreover,

$$
R=d\left(x, U^{\prime}\right) \leqslant K\left[d\left(x, x_{j}\right)+d\left(x_{j}, U^{\prime}\right)\right]<K\left[C s_{j}+2 C K s_{j}\right]
$$

and it follows that $s_{j} \geqslant R /(1+2 K) C K$.

If $x$ also belong to $\bar{S}_{i}, i \neq j$, the same argument shows $\bar{S}_{i} \subset S_{2 K R}(x)$. Thus, all such points $x_{i}, x_{j}, \ldots$ belong to $S_{2 K R}(x)$. But $S_{r\left(x_{i}\right)}\left(x_{i}\right) \cap S_{r\left(x_{j}\right)}\left(x_{j}\right)$ $=\varnothing$ when $i \neq j$ and this implies

$$
d\left(x_{i}, x_{j}\right) \geqslant \min \left\{r\left(x_{i}\right), r\left(x_{j}\right)\right\}=\frac{1}{4 K} \min \left\{s_{i}, s_{j}\right\} \geqslant \frac{R}{4 C K^{2}(1+2 K)} .
$$

It now follows from (3.4) that there cannot be more than $M$ $\leqslant N N^{\log _{2} 4 C K^{2}(1+2 K)}$ spheres $\bar{S}_{j}$ containing $x$ and (ii) is established.

Property (ii) will be referred to as the $M$-disjointness of the collection $\left\{\bar{S}_{j}\right\}$.

We shall make repeated use of the following extension to spaces of homogeneous type of the Hardy-Littlewood maximal function

$$
(\mathfrak{M} f)(x)=\sup \left\{\frac{1}{\mu(S)} \int_{S}|f| d \mu: S \text { is a sphere, } x \in S\right\} .
$$

One can show, by using (3.1), that $\mathfrak{M} f$ is defined and finite a.e. for $f \in L^{1}(X)$; moreover, we have the weak-type inequality:

Theorem (3.5). If $f \in L^{1}(X)$ there exists a constant $C_{0}=C_{0}(A, K)$ such that

$$
\mu(\{x \in X:(\mathfrak{M} f)(x)>\alpha\}) \leqslant\left(C_{0} / \alpha\right)\|f\|_{1} .
$$

(See Theorem (2.1) on p. 71 of [17].)

From this result and the obvious boundedness of this maximal operator on $L^{\infty}$ one establishes the existence of a constant $A_{p}$ such that

$$
\|\mathfrak{M} f\|_{p} \leqslant A\|f\|_{p}
$$

when $p>1$ (one can prove this directly as is done in the second chapter of [57] or one can appeal to the Marcinkiewicz interpolation theorem).

A useful variant of the Hardy-Littlewood maximal operator is $\mathfrak{M}_{p_{0}} f$ $=\left(\mathfrak{M}|f|^{p_{0}}\right)^{1 / p_{0}}$. From (3.5) and (3.6) we obtain the inequalities

$$
\mu\left(\left\{x:\left(\mathfrak{M}_{p_{0}} f\right)(x)>\alpha\right\}\right) \leqslant C_{0}\left(\|f\|_{p_{0}} / \alpha\right)^{p_{0}}
$$

and, for $p>p_{0}$,

$$
\left\|\mathfrak{M}_{p_{0}} f\right\|_{p} \leqslant A_{\left(p / p_{0}\right)}^{1 / p_{0}}\|f\|_{p} .
$$


It is often useful to use the centered maximal function of $f$ :

$$
\left(\mathfrak{M}_{c} f\right)(x)=\sup _{r>0} \frac{1}{\mu\left(S_{r}(x)\right)} \int_{S_{r}(x)}|f| d \mu .
$$

The operators $\mathfrak{M}$ and $\mathfrak{M}_{c}$ are "equivalent" in the sense that

$$
\left(\mathfrak{M}_{c} f\right)(x) \leqslant(\mathfrak{M} f)(x) \leqslant D\left(\mathfrak{M}_{c} f\right)(x),
$$

where $D=D(A, K)$. This follows from the simple observation that if $x$ $\in S_{r}(y)$ then $S_{r}(y) \subset S_{2 K r}(x) \subset S_{K(1+2 K) r}(y)$. Thus,

$$
\frac{1}{\mu\left(S_{r}(y)\right)} \int_{S_{r}(y)}|f| d \mu \leqslant\left\{\frac{\mu\left(S_{K(1+2 K) r}(y)\right.}{\mu\left(S_{r}(y)\right)}\right\} \frac{1}{\mu\left(S_{2 K r}(x)\right)} \int_{S_{2 K_{r}}(x)}|f| d \mu
$$

and we obtain (3.9) by making use of inequality (2.1).

The centered maximal function is useful, for example, in the proof the following geometric result:

Lemma (3.9). If $f \in L^{1}(X)$ has support in $S_{0}=S_{r_{0}}\left(x_{0}\right)$ then there exists $C_{1}=C_{1}(A, K)$ such that

$$
U^{\alpha}=\{x \in X:(\mathfrak{M} f)(x)>\alpha\} \subset S_{2 K_{0}}\left(x_{0}\right)
$$

whenever $\alpha>C_{1} m_{S_{0}}(|f|)=C_{1}\left(1 / \mu\left(S_{0}\right)\right) \int_{S_{0}}|f| d \mu$.

Proof. We first observe that if $d\left(x, x_{0}\right) \geqslant 2 K r_{0}$ and $r \leqslant r_{0}$ then $S_{r}(x) \cap S_{0}$ $=\varnothing$. To see this, suppose $y \in S_{r}(x) \cap S_{0}$. Then

$$
2 K r_{0} \leqslant d\left(x, x_{0}\right) \leqslant K\left[d(x, y)+d\left(y, x_{0}\right)\right]<K\left[r+r_{0}\right] \leqslant 2 K_{0}
$$

which shows that such a $y$ cannot exist. On the other hand, if $r=d\left(x, x_{0}\right)$ $\geqslant r_{0}$ then $S_{0} \subset S_{2 K r}(x)$. This is also immediate: if $y \in S_{0}$

$$
d(x, y) \leqslant K\left[d\left(x, x_{0}\right)+d\left(x_{0}, y\right)\right]<K\left[r+r_{0}\right] \leqslant 2 K r .
$$

From the first observation we see that if $d\left(x, x_{0}\right) \geqslant 2 K r_{0}$ then $\int_{S_{r}(x)}|f| d \mu$ $=0$ if $r \leqslant r_{0}$. Thus, the centered maximal function of $f$ satisfies

$$
\left(\mathfrak{M}_{c} f\right)(x)=\sup _{r \geqslant r_{0}} \frac{1}{\mu\left(S_{r}(x)\right)} \int_{S_{r}(x)}|f| d \mu .
$$

Since $f$ has support in $S_{0}$ we have

$$
\frac{1}{\mu\left(S_{r}(x)\right)} \int_{S_{r}(x)}|f| d \mu \leqslant\left\{\frac{\mu\left(S_{0}\right)}{\mu\left(S_{r}(x)\right)}\right\} \frac{1}{\mu\left(S_{0}\right)} \int_{S_{0}}|f| d \mu .
$$

If $r \geqslant r_{0}$, the second observation implies (we also use (2.1))

$$
\frac{\mu\left(S_{0}\right)}{\mu\left(S_{r}(x)\right)} \leqslant \frac{\mu\left(S_{2 K r}(x)\right)}{\mu\left(S_{r}(x)\right)} \leqslant A^{2+\log _{2} K}=C .
$$

Thus, if $r \geqslant r_{0}, m_{S_{r}(x)}(|f|) \leqslant C m_{S_{0}}(|f|)$ and we see that if $\beta>C m_{S_{0}}(|f|)$ 
then $\left\{x:\left(\mathfrak{M}_{c} f\right)(x)>\beta\right\} \subset S_{2 r_{0}}(x)$. By (3.9) it now follows that if $\alpha$ $>D C m_{S_{0}}(|f|) \equiv C_{1} m_{S_{0}}(|f|)$ then $U^{\alpha} \subset S_{2 K r_{0}}(x)$ and the lemma is proved.

We are now ready to prove Theorem $A$. We have already observed that $H^{p, q} \supset H^{p, \infty}$ for $0<p \leqslant 1 \leqslant q, p<q$; thus, we need to establish the opposite inclusion relation. We shall do so by showing that a $(p, q)$-atom $a(x)$ has the representation

$$
a=\sum_{j} \alpha_{j} a_{j}
$$

where each $a_{j}$ is a $(p, \infty)$-atom and $\sum\left|\alpha_{j}\right|^{p} \leqslant B, B$ independent of $a$. More explicitly, we shall show that the linear functionals $a$ and $\sum_{j} \alpha_{j} a_{j}$, acting on $\mathfrak{L}_{(1 / p)-1}$, are equal when $p<1$; when $p=1(3.10)$ is an equality in $L^{1}(X)$. We shall give an inductive proof. In order to state the induction hypothesis we need to introduce some notation:

For each positive integer $n$ let $N^{n}$ denote the $n$-fold cartesian product of the natural numbers $N, N^{0} \equiv\{0\}$. We write $j_{n}$ to represent the general element of $N^{n}$, the support of $a$ is contained in the sphere $S_{0}$ (the three defining properties of a $(p, q)$-atom are satisfied with respect to $\left.S_{0}\right)$, and we set $b=\left[\mu\left(S_{0}\right)\right]^{1 / p} a$. We shall apply Theorem (3.2) with the constant $C=2 K$; thus, the $M$ occurring in part (ii) of (3.2) depends only on $A$ and $K$. From (2.1) we can, therefore, deduce that the ratio $\mu\left(\bar{S}_{j}\right) / \mu\left(S_{j}\right)$ does not exceed some fixed constant $k_{0}=k_{0}(A, K)$. Given a sphere $S$ of radius $s, \bar{S}$ and $\bar{S}$ will denote spheres with the same center as $S$ having radii $2 K s$ and $6 K^{2} s$ respectively.

The induction hypothesis we shall establish is the following one:

There exists a collection of spheres $\left\{S_{j_{l}}\right\}, j_{l} \in N^{l}$ for $l=0,1, \ldots$, such that for each natural number $n$

$$
b=M k_{0} \alpha \sum_{l=0}^{n-1} \alpha^{l} \sum_{j_{l} \in N^{l}}\left[\mu\left(\bar{S}_{j_{l}}\right)\right]^{1 / p} a_{j_{l}}+\sum_{j_{n} \in N^{n}} h_{j_{n}},
$$

where $\alpha=\alpha(p, q, A, K)$ is sufficiently large (we shall be explicit later) and

(I) $a_{j_{l}}$ is a $(p, \infty)$-atom supported in $\bar{S}_{j_{l}}, l=0,1, \ldots, n-1$;

(II) $\cup_{j_{n} \in N^{n}} S_{j_{n}} \subset\left\{x:\left(\mathfrak{M}_{q} b\right)(x)>\alpha^{h} / 2\right\}$;

(III) $\left\{S_{j_{l}}\right\}$ is an $M^{l}$-disjoint collection;

(IV) the functions $h_{j_{n}}$ are supported in $S_{j_{n}}$;

(V) $\int h_{j_{n}} d \mu=0$;

(VI) $\left|h_{j_{n}}(x)\right| \leqslant|b(x)|+2 k_{0}^{1 / q} \alpha^{n} \chi_{S_{j_{n}}}(x)$;

(VII) $\left[m_{S_{j_{n}}}\left(\left|h_{j_{n}}\right|^{q}\right)\right]^{1 / q} \leqslant 2 k_{0}^{1 / q} \alpha^{n}$.

Let us first show that if these properties are valid for each $n \in N$ then (3.10) holds. We begin by proving

$$
\frac{\left(M k_{0} \alpha\right)^{p}}{\mu\left(S_{0}\right)} \sum_{n=0}^{\infty} \alpha^{n p} \sum_{j_{n} \in N^{n}} \mu\left(\bar{S}_{j_{n}}\right) \leqslant B,
$$

where $B$ is independent of $a$. From (II) and (III) we have

$$
\begin{aligned}
\sum_{j_{n} \in N^{n}} \mu\left(\bar{S}_{j_{n}}\right) & \leqslant k_{0} \sum_{j_{n} \in N^{n}} \mu\left(S_{j_{n}}\right) \leqslant k_{0} M^{n} \mu\left(\bigcup_{i \in N^{n}} S_{j_{n}}\right) \\
& \leqslant k_{0} M^{n} \mu\left(\left\{x:\left(\mathfrak{M}_{q} b\right)(x)>\alpha^{n} / 2\right\}\right) .
\end{aligned}
$$


From (3.7) we thus deduce that

$$
\sum_{j_{n} \in N^{n}} \mu\left(\bar{S}_{j_{n}}\right) \leqslant C_{0} k_{0} M^{n}\left(\frac{2}{\alpha^{n}}\right)^{q}\|b\|_{q}^{q} .
$$

Consequently,

$$
\sum_{n=0}^{\infty} \alpha^{n p} \sum_{j_{n} \in N^{n}} \mu\left(\bar{S}_{j_{n}}\right) \leqslant C_{0} k_{0} 2^{q} \sum_{n=0}^{\infty}\left(M \alpha^{p-q}\right)^{n}\|b\|_{q}^{q} .
$$

Since $\|b\|_{q}^{q} \leqslant \mu\left(S_{0}\right)$ we obtain (3.12) with $B$ depending on $p, q, A, K$ and $\alpha$ (as long as $\left.\alpha^{p-q} M<1\right)$ but not on $a$.

From (VII) we have

$$
\int\left|h_{j_{n}}\right| d \mu \leqslant \mu\left(S_{j_{n}}\right) 2 k_{0}^{1 / q} \alpha^{n}=C \alpha^{n} \mu\left(S_{j_{n}}\right)
$$

thus, if we let $H_{n}=\sum_{j_{n} \in N^{n}} h_{j_{n}}$, using the $M^{n}$-disjointness of $\left\{S_{j_{n}}\right\}$ and the above estimate for $\sum \mu\left(S_{j_{n}}\right)$ we see that

$$
\int\left|H_{n}\right| d \mu \leqslant \sum_{j_{n} \in N^{n}} \int\left|h_{j_{n}}\right| d \mu \leqslant C \alpha^{n} \sum_{j_{n} \in N^{n}} \mu\left(S_{j_{n}}\right) \leqslant C\left(M \alpha^{1-q}\right)^{n}\|b\|_{q}^{q} .
$$

This shows that if $p=1$ (and, thus, $q>1$ ) the first series on the right-hand side of (3.11) converges to $b$ in $L^{1}(X)$. Equality (3.11) is to be interpreted in the following way when $p<1$ : If $l \in \mathfrak{L}_{\beta}, \beta=1 / p-1$, then

$$
\int b l d \mu=M k_{0} \alpha \sum_{k=0}^{n-1} \alpha^{k} \sum_{j_{k} \in N^{k}}\left[\mu\left(\bar{S}_{j_{k}}\right)\right]^{1 / p} \int a_{j_{k}} l d \mu+\sum_{j_{n} \in N^{n}} h_{j_{n}} l d \mu .
$$

Inequality (3.12) assures us that the double sum on the right is well defined. The fact that $\left\|H_{n}\right\|_{1} \leqslant \Sigma\left\|h_{j_{n}}\right\|_{1}<\infty$ and $H_{n}$ has bounded support (this follows from (II), (IV) and (3.9) if $\alpha^{q}>C_{1}$ ) imply that the last sum equals $\int H_{n} l d \mu$ and is well defined. Theorem $\mathrm{A}$ is then proved if we show that $\lim _{n \rightarrow \infty} \int H_{n} l d \mu=0$. To see that this is true let $x_{j_{n}}$ be the center of $S_{j_{n}}$ and suppose $\|l\|^{(\beta)}=1$. Then, using (V) and (VII) and the above estimate for $\sum \mu\left(S_{j_{n}}\right)$

$$
\begin{aligned}
\left|\int H_{n} l d \mu\right| & =\sum_{j_{n} \in N^{n}}\left|\int_{S_{j_{n}}} h_{j_{n}} l d \mu\right| \leqslant \Sigma\left|\int_{S_{j_{n}}} h_{j_{n}}\left(l-l\left(x_{j_{n}}\right)\right) d \mu\right| \\
& \leqslant \sum\left[\mu\left(S_{j_{n}}\right)\right]^{-1+1 / p} \int_{S_{j_{n}}}\left|h_{j_{n}}\right| d \mu \leqslant C \alpha^{n} \sum\left[\mu\left(S_{j_{n}}\right)\right]^{1 / p} \\
& \leqslant C \alpha^{n}\left[\left(M \alpha^{-q}\right)^{n}\|b\|_{q}^{q}\right]^{1 / p}=C\|b\|_{q}^{q / p}\left(M \alpha^{p-q}\right)^{n / p} .
\end{aligned}
$$

Since $p<q$ the last term tends to 0 and the theorem is proved if the induction hypothesis is established.

Let us show that the hypothesis is valid for $n=1$. Let $U^{\alpha}=$ $\left\{x \in X:\left(\mathfrak{M}_{q} b\right)(x)>\alpha\right\}$. By (3.9) $U^{\alpha} \subset \bar{S}_{0}$ provided $\alpha^{q}>C_{1}$. Assuming this condition on $\alpha$, we see that $U^{\alpha}$ is a bounded open set. By (3.7) we have

$$
\mu\left(U^{\alpha}\right) \leqslant C_{0}\left(\|b\|_{q} / \alpha\right)^{q} \leqslant C_{0} \alpha^{-q} \mu\left(S_{0}\right) .
$$


Hence, if $\alpha^{q}>C_{0}$ (which we assume to be the case) $\mu\left(U^{\alpha}\right)<\mu\left(S_{0}\right) \leqslant \mu(X)$ and $X-U^{\alpha}$ cannot be empty. We can, therefore, apply Theorem (3.2) and obtain a Whitney-type covering of $U^{\alpha}$ by spheres $\left\{S_{j}\right\}$ satisfying conditions (i), (ii) and (iii) with $C=2 K$. Letting $\chi_{j}$ be the characteristic function of $S_{j}$ we define

$$
\eta_{j}(x)= \begin{cases}\frac{\chi_{j}(x)}{\Sigma \chi_{i}(x)} & \text { if } x \in U^{\alpha}, \\ 0 & \text { if } x \notin U^{\alpha}\end{cases}
$$

(observe that the $M$-disjointness of $\left\{S_{j}\right\}$ implies that $\Sigma \chi_{i}(x)$ is a finite sum for each $\left.x \in U^{\alpha}\right)$. We then put

$$
g_{0}(x)= \begin{cases}b(x) & \text { if } x \notin U^{\alpha} \\ \sum m_{S_{j}}\left(\eta_{j} b\right) x_{j}(x) & \text { if } x \in U^{\alpha}\end{cases}
$$

and $h_{j}(x)=b(x) \eta_{j}(x)-m_{S_{j}}\left(\eta_{j} b\right) \chi_{j}(x)$ for all $x \in X$. It follows that

$$
b(x)=g_{0}(x)+\sum_{j \in N} h_{j}(x)
$$

and we shall show that, by an appropriate normalization, this is the representation (3.11) of $b$ when $n=1$. This equality is a version of the CalderónZygmund decomposition of the function $b$ into the "good" function $g_{0}$ and "bad" function $H=\Sigma h_{j}$. The induction proof we are presenting will show how we can keep refining this decomposition by applying it repeatedly to the bad function.

If $x \notin U^{\alpha}$ then $\left|g_{0}(x)\right|=|b(x)| \leqslant\left(\mathfrak{M}_{q} b\right)(x) \leqslant \alpha,{ }^{30}$ while if $x \in U^{\alpha}$

$$
\begin{aligned}
\left|g_{0}(x)\right| & \leqslant \sum_{\substack{\text { at most } \\
M \text { terms }}} \frac{1}{\mu\left(S_{j}\right)} \int_{S_{j}}\left|b \eta_{j}\right| d \mu \leqslant \sum_{\text {ibid }} \frac{\mu\left(\overline{\bar{S}_{j}}\right)}{\mu\left(S_{j}\right)}\left[\frac{1}{\mu\left(\overline{\bar{S}_{j}}\right)} \int_{\overline{\bar{S}_{j}}}|b|^{q} d \mu\right]^{1 / q} \leqslant \sum_{\text {ibid }} k_{0} \alpha \\
& \leqslant\left(M k_{0}\right) \alpha .
\end{aligned}
$$

This shows that

(1) $\left|g_{0}(x)\right| \leqslant\left(M k_{0}\right) \alpha$.

We have already observed that $U^{\alpha} \subset \bar{S}_{0}$; for $x \notin U^{\alpha}, g_{0}(x)=b(x)$. These facts, together with the inclusions Supp $b \subset S_{0} \subset \bar{S}_{0}$ imply

(2) the support of $g_{0}$ is within $\bar{S}_{0}$.

We also clearly have:

(3) the support of $h_{j}$ is within $S_{j}$;

(4) $\int h_{j} d \mu=0$.

Since $\left\|h_{j}\right\|_{1} \leqslant 2\left\|b \chi_{j}\right\|_{1}=2 \int_{S_{j}}|b| d \mu$ and the spheres $S_{j}$ are $M$-disjoint we must have

$$
\begin{aligned}
\sum_{j}\left\|h_{j}\right\|_{1} & \leqslant 2 \sum_{j} \int_{S_{j}}|b| d \mu \leqslant 2 M \int_{U^{\alpha}}|b| d \mu \leqslant 2 M\|b\|_{1} \\
& \leqslant 2 M\|b\|_{q}\left[\mu\left(S_{0}\right)\right]^{1 / q^{\prime}} \leqslant 2 M \mu\left(S_{0}\right) .
\end{aligned}
$$

${ }^{30}$ That $|b(x)| \leqslant\left(\mathfrak{M}_{q} b\right)(x)$ holds for almost every $x$ is true if we assume that $\mu$ is a regular measure. This assumption is tacitly made throughout this paper. 
In particular, this shows that the sum in (3.13) is convergent in the $L^{\mathrm{I}}$-norm; from (4) and $\int b d \mu=0$ we thus can conclude that

(5) $\int g_{0} d \mu=0$.

It follows that $a_{0} \equiv g_{0} / M k_{0} \alpha\left[\mu\left(\bar{S}_{0}\right)\right]^{1 / p}$ is a $(p, \infty)$-atom. Thus, we can write (3.13) in the form

$$
b=M k_{0} \alpha\left[\mu\left(\bar{S}_{0}\right)\right]^{1 / p} a_{0}+\sum_{j \in N} h_{j}
$$

which is the special case $n=1$ of (3.11). Moreover, property (I) has just been established. (3) and (4) are parts (IV) and (V) of the induction hypothesis when $n=1$. Since

$$
\bigcup_{j} S_{j}=U^{\alpha}=\left\{x:\left(\mathfrak{M}_{q} b\right)(x)>\alpha\right\} \subset\left\{x:\left(\mathfrak{M}_{q} b\right)(x)>\alpha / 2\right\}
$$

property (II) is also satisfied. Property (III) is a consequence of the Whitneytype covering we are using (property (ii) of (3.2)). Since $\eta_{j}(x) \leqslant 1$ we have

$$
\left|h_{j}(x)\right| \leqslant|b(x)|+\left|m_{S_{j}}\left(\eta_{j} b\right)\right| \chi_{j}(x) \leqslant|b(x)|+k_{0}^{1 / q} \alpha \chi_{j}(x)
$$

and, thus, property (VI) holds. Similarly, we obtain (VII):

$$
\begin{aligned}
\left(\frac{1}{\mu\left(S_{j}\right)} \int_{S_{j}}\left|h_{j}\right|^{q} d \mu\right)^{1 / q} & \leqslant\left(\frac{1}{\mu\left(S_{j}\right)} \int_{S_{j}}|b|^{q} d \mu\right)^{1 / q}+\alpha k_{0}^{1 / q} \\
& \leqslant\left(\frac{\mu\left(\bar{S}_{j}\right)}{\mu\left(S_{j}\right)} \frac{1}{\mu\left(\overline{\bar{S}_{j}}\right)} \int_{\overline{\bar{S}}_{j}}|b|^{q} d \mu\right)^{1 / q}+\alpha k_{0}^{1 / q} \leqslant 2 \alpha k_{0}^{1 / q} .
\end{aligned}
$$

This shows that the induction hypothesis holds for $n=1$. We now assume it true for $n$ and will show that this implies its validity for $n+1$.

Let $U_{j_{n}}=\left\{x \in X:\left(\mathfrak{M}_{q} h_{j_{n}}\right)(x)>\alpha^{n+1}\right\}$. Hypothesis (IV) tells us that the support of $h_{j_{n}}$ is within $S_{j_{n}}$. It follows from (VII), provided $\alpha^{q}>2^{q} k_{0} C_{1}$, that

$$
C_{1} m_{S_{j_{n}}}\left(\left|h_{j_{n}}\right|^{q}\right) \leqslant C_{1} k_{0}\left(2 \alpha^{n}\right)^{q}<\alpha^{q(n+1)} .
$$

From Lemma (3.9), therefore, we obtain

$$
U_{j_{n}}=\left\{x \in X:\left(\mathfrak{M}\left|h_{j_{n}}\right|^{q}\right)(x)>\alpha^{q(n+1)}\right\} \subset \bar{S}_{j_{n}} .
$$

Let $\left\{S_{j_{n}, i}\right\}$ be a Whitney covering for $U_{j_{n}}$ with constant $C=2 K$ (see Theorem (3.2)). From (3.3) and (3.2)(ii) we have $\bigcup_{i} \bar{S}_{j_{n}, i}=U_{j_{n}} \subset \bar{S}_{j_{n}}$ and $\left\{\bar{S}_{j_{n}, i}\right\}$ is $M$ disjoint. Since, from (III), we know that $\left\{\bar{S}_{j_{n}}\right\}$ are $M^{n}$-disjoint it follows that the totality of spheres in the family $\left\{\bar{S}_{j_{n}, i}\right\}$ are $M^{n+1}$ disjoint. This establishes hypothesis (III) for $n+1$.

We now put

$$
g_{j_{n}}(x)= \begin{cases}h_{j_{n}}(x) & \text { if } x \notin U_{j_{n}}, \\ \sum_{i} m_{S_{j_{n}, i}}\left(h_{j_{n}} \eta_{j_{n}}^{i}\right) \chi_{S_{j_{n},}}(x) & \text { if } x \in U_{j_{n}},\end{cases}
$$

and 


$$
h_{j_{n}, i}=h_{j_{n}} \eta_{j_{n}}^{i}-m_{S_{j_{n}, i}}\left(h_{j_{n}} \eta_{j_{n}}^{i}\right) \chi_{S_{j_{n}, i}}(x)
$$

where $\eta_{j_{n}}^{i}(x)=\chi_{s_{j_{n},}}(x) / \Sigma_{l} \chi_{S_{j_{n}, l}}(x)$ for $x \in U_{j_{n}}$ and is 0 if $x \notin U_{j_{n}}$. If $x \in U_{j_{n}}$, therefore,

$$
\left|g_{j_{n}}(x)\right| \leqslant \sum_{\substack{\text { at most } \\ M \text { terms }}}\left|m_{S_{j_{n}, i}}\left(h_{j_{n}} \eta_{j_{n}}^{i}\right) \chi_{j_{n}}^{i}(x)\right| \leqslant k_{0} M \alpha^{n+1}
$$

while, if $x \in U_{j_{n}}$ then $\left|g_{j_{n}}(x)\right|=\left|h_{j_{n}}(x)\right| \leqslant\left(\mathfrak{M}_{q} h_{j_{n}}\right)(x) \leqslant \alpha^{n+1}$. In any case $\left\|g_{j_{n}}\right\|_{\infty} \leqslant k_{0} M \alpha^{n+1}$. Since the support of $h_{j_{n}}$ is within $S_{j_{n}} \subset \bar{S}_{j_{n}}$ and $U_{j_{n}} \subset \bar{S}_{j_{n}}$ it follows that the support of $g_{j_{n}}$ is within $S_{j_{n}}$. Moreover, $\int h_{j_{n}, i} d \mu=0$ (which shows that property $(\mathrm{V})$ is valid for $n+1)$. In the same way we showed that $\sum\left\|h_{j}\right\|_{1} \leqslant 2 M\|b\|_{1}$ when the case $n=1$ was considered, we can show $\sum_{i}\left\|h_{j_{n}, i}\right\|_{1} \leqslant 2 M\left\|h_{j_{n}}\right\|_{1}$. It follows, therefore, that

$$
h_{j_{n}}=g_{j_{n}}+\sum_{i} h_{j_{n}, i}
$$

is valid in $L^{1}(X)$ (it is also true a.e. since, for each $x$, the sum on the right has at most $M$ terms) and $\int g_{j_{n}} d \mu=0$. Hence

$$
a_{j_{n}}=g_{j_{n}} /\left[k_{0} M \alpha^{n+1}\left(\mu\left(\bar{S}_{j_{n}}\right)\right)^{1 / p}\right]
$$

is a $(p, \infty)$-atom supported in the sphere $\bar{S}_{j_{n}}$. From this it follows that the representation (3.11) is true for $n+1$ and so is (I). Property (IV) is trivially true. We have, from the definition of $h_{j_{n}, i}$ and (VI),

$$
\begin{aligned}
\left|h_{j_{n}, i}(x)\right| & \leqslant\left\{\left|h_{j_{n}}(x)\right|+\left[k_{0} \frac{1}{\mu\left(\overline{\bar{S}}_{j_{n}, i}\right)} \int_{\overline{\bar{S}}_{j_{n}, i}} \mid h_{j_{n}}{ }^{q} d \mu\right]^{1 / q}\right\} x_{j_{n}}^{i}(x) \\
& \leqslant\left\{|b(x)|+2 k_{0}^{1 / q} \alpha^{n}+k_{0}^{1 / q} \alpha^{n+1}\right\} \chi_{j_{n}}^{i}(x) \\
& \leqslant\left\{|b(x)|+2 k_{0}^{1 / q} \alpha^{n+1}\right\} \chi_{j_{n}}^{i}(x)
\end{aligned}
$$

as long as $\alpha>2$. This establishes (VI) for $n+1$. Property (VII) has a similar proof: from the definition of $h_{j_{n}, i}$

$$
\begin{aligned}
{\left[m_{S_{j_{n}, i}}\left(\mid h_{j_{n},} i^{q}\right)\right]^{1 / q} } & \leqslant 2\left[m_{S_{j_{n}, i}}\left(\left|h_{j_{n}}\right|^{q}\right)\right]^{1 / q} \\
& \leqslant 2\left[k_{0} m_{\bar{S}_{j_{n}, i}}\left(\left|h_{j_{n}}\right|^{q}\right)\right]^{1 / q} \leqslant 2 k_{0}^{1 / q} \alpha^{n+1} .
\end{aligned}
$$

Finally, we establish (II): From (VI) we have $\left(\mathfrak{M}_{q} h_{j_{n}}\right)(x) \leqslant\left(\mathfrak{M}_{q} b\right)(x)$ $+2 k_{0}^{1 / q} \alpha^{n}$. Thus, if $x \in U_{j_{n}}$

$$
\alpha^{n+1}<\left(\mathfrak{M}_{q} h_{j_{n}}\right)(x) \leqslant\left(\mathfrak{M}_{q} b\right)(x)+2 k_{0}^{1 / q} \alpha^{n} .
$$

It follows that $\alpha^{n+1} / 2<\left(\mathfrak{M}_{q} b\right)(x)$ if $\alpha>4 k_{0}^{1 / q}$ and, thus, 


$$
\bigcup_{j_{n}, i} S_{j_{n}, i}=\bigcup_{j_{n}}\left(\bigcup_{i} S_{j_{n}, i}\right) \subset \bigcup_{j_{n}} U_{j_{n}} \subset\left\{x \in X:\left(\mathfrak{M}_{q} b\right)(x)>\frac{\alpha^{n+1}}{2}\right\}
$$

and (II) is valid for $n+1$.

Observe that we used the fact that $\alpha$ exceeds each of the following numbers: $4 k_{0}^{1 / q}, 2,2\left(k_{0} C_{1}\right)^{1 / q}, C_{0}^{1 / q}, M^{1 /(q-p)}, M^{1 /(q-1)}$. Each of these numbers depends on $p, q, A$ and $K$ and are independent of $n$. Theorem A is, therefore, proved.

Let us now pass to the study of the dual space of $H^{p}(X)$. We begin with the case $p=1$ and, toward this end, we first make the following observation:

Lemma (3.14). If $b \in B M O_{q}, 1 \leqslant q<\infty$, then $|b| \in B M O_{q}$.

In order to see this we note that if a function $b$ satisfies: for each sphere $S$ there is a constant $b_{S}$ such that

$$
\left\{\frac{1}{\mu(S)} \int_{S}\left|b(x)-b_{S}\right|^{q} d \mu\right\}^{1 / q} \leqslant C,
$$

where $C$ is independent of $S$ then $b \in B M O_{q}$ and $\|b\|_{B M O}^{(q)}$ does not exceed $2 C$, if $\mu(X)=\infty$, or $2 C+\left|\int_{X} b d \mu\right|$ if $\mu(X)=1$. This follows from

$$
\begin{aligned}
\left\{\frac{1}{\mu(S)} \int_{S}\left|b-m_{S}(b)\right|^{q} d \mu\right\}^{1 / q} \leqslant & \left\{\frac{1}{\mu(S)} \int_{S}\left|b-b_{S}\right|^{q} d \mu\right\}^{1 / q} \\
& +\left\{\frac{1}{\mu(S)} \int_{S}\left|b_{S}-m_{S}(b)\right|^{q} d \mu\right\}^{1 / q} \\
\leqslant & +\left|\int_{S}\left(b_{S}-b(x)\right) \frac{d \mu(x)}{\mu(S)}\right|^{1 / q} \leqslant 2 C . \\
\leqslant & +\left\{\int_{S}\left|b_{S}-b(x)\right|^{q} \frac{d \mu(x)}{\mu(S)}\right\}^{1 / q} \leqslant 2 C
\end{aligned}
$$

Lemma (3.14) now follows from this and the inequality

$$
|| b(x)|-| m_{S}(b)|| \leqslant\left|b(x)-m_{S}(b)\right| .
$$

Since the infimum (supremum) of two numbers $x$ and $y$ is

$$
(x+y-|x-y|) / 2((x+y+|x-y|) / 2)
$$

it follows immediately from (3.14) that $B M O_{q}$ is a lattice. In particular, the function

$$
b_{N}(x)= \begin{cases}N & \text { if } b(x) \geqslant N, \\ b(x) & \text { if }-N<b(x)<N, \\ -N & \text { if } b(x) \leqslant-N,\end{cases}
$$

belongs to $B M O_{q}$ if $b$ does. Recall that $\|b\|_{B M O}^{(q)}$ equals $\mathfrak{N}_{q}(b)$, if $\mu(X)=\infty$, or $\mathfrak{N}_{q}(b)+\left|\int b d \mu\right|$, if $\mu(X)=1$. It follows from the above considerations that 


$$
\left\|b_{N}\right\|_{B M O}^{(q)} \leqslant \begin{cases}3 \mathfrak{N}_{q}(b) & \text { if } \mu(X)=\infty \\ 3 \mathfrak{N}_{q}(b)+\left|\int b_{N} d \mu\right| & \text { if } \mu(X)=1 .\end{cases}
$$

Thus, in either case, we have (from the dominated convergence theorem)

$$
\left\|b_{N}\right\|_{B M O}^{(q)} \leqslant 4\|b\|_{B M O}^{(q)}
$$

provided $N$ is large enough.

Let $q^{\prime}$ be the conjugate index to $q:\left(1 / q^{\prime}\right)+(1 / q)=1$. We assume $1 \leqslant q$ $<\infty$ and, thus, $1<q^{\prime} \leqslant \infty$. Suppose $f=\sum \alpha_{j} a_{j}$ belongs to $H^{1, q^{\prime}}$, where $a_{j}$ is a $\left(1, q^{\prime}\right)$-atom supported in the sphere $S_{j}, \sum\left|\alpha_{j}\right|<\infty$ and $b$ is a bounded function on $X$. Then, assuming $\int a_{j} d \mu=0$ for all $j$ (which is always true if $\mu(X)=\infty)$.

$$
\begin{aligned}
\left|\int f b d \mu\right| & \leqslant \Sigma\left|\alpha_{j}\right|\left|\int a_{j} b d \mu\right|=\Sigma\left|\alpha_{j}\right|\left|\int a_{j}\left(b-m_{S_{j}}(b)\right) d \mu\right| \\
& \leqslant \Sigma\left|\alpha_{j}\right|\left(\int\left|a_{j}\right|^{q^{\prime}} d \mu\right)^{1 / q^{\prime}}\left(\int_{S_{j}}\left|b(x)-m_{S_{j}}(b)\right|^{q} d \mu(x)\right)^{1 / q} \\
& \leqslant \Sigma\left|\alpha_{j}\right|\left(\frac{1}{\mu\left(S_{j}\right)} \int_{S_{j}}\left|b(x)-m_{S_{j}}(b)\right|^{q} d \mu(x)\right)^{1 / q} \leqslant\left(\Sigma\left|\alpha_{j}\right|\right) \Re_{q}(b) .
\end{aligned}
$$

From this we can easily deduce $\left|\int f b d \mu\right| \leqslant\|f\|_{\left(1, q^{\prime}\right)}\|b\|_{B M O}^{(q)}$ whenever $f$ $\in H^{1, q^{\prime}}$ and $b \in L^{\infty}$. From this last inequality and (3.15), therefore, we obtain

$$
\left|\int f b_{N} d \mu\right| \leqslant 4\|f\|_{\left(1, q^{\prime}\right)}\|b\|_{B M O}^{(q)}
$$

whenever $f \in H^{1, q^{\prime}}, b \in B M O_{q^{\prime}}$ and $N$ is sufficiently large.

Let $L^{q^{\prime}}=\left\{f \in L^{q^{\prime}}(X)\right.$ : Support of $f$ is bounded $\}$ and put $\mathscr{D}=H^{1, q^{\prime}}$ $\cap L_{0}^{g^{\prime}}$. Then, the linear functional $L_{b}$ mapping $f$ into $\int f b d \mu$ is well defined on D whenever $b \in B M O_{q}$ (since $b$ is locally in $L^{q}$ ). By the dominated convergence theorem,

$$
\lim _{N \rightarrow \infty} \int f b_{N} d \mu=\int f b d \mu
$$

for $f \in \mathscr{D}$. This equality and (3.16) give us $\left|L_{b} f\right| \leqslant 4\|f\|_{\left(1, q^{\prime}\right)}\|b\|_{B M O}^{(q)}$ for all $f \in \mathscr{D}$ and $b \in B M O_{q}$. But $\mathscr{D}$ is dense in $H^{(1, q)}$ (a partial sum $\sum_{1}^{n} \alpha_{j} a_{j}$ of an atomic representation $f=\sum \alpha_{j} a_{j}$ with $\sum\left|\alpha_{j}\right| \leqslant(1+\varepsilon)\|f\|_{1, q^{\prime}}$ can be used to approximate an $f \in H^{1, q^{\prime}}$ by elements of $\left.\mathscr{D}\right)$ ). Thus, $L_{b}$ has a unique bounded extension on $H^{1, q^{\prime}}$ which we also denote by $L_{b}$. In this sense we have

$$
B M O_{q} \subset\left(H^{1, q^{\prime}}\right)^{*}
$$

and the linear functional norm $\left\|L_{b}\right\|, b \in B M O_{q}$, satisfies

$$
\left\|L_{b}\right\| \leqslant 4\|b\|_{B M O}^{(q)}
$$


Now suppose $L$ is a bounded linear functional on $H^{1, q^{\prime}}$. Let $\|L\|$ be the norm of $L$. If $S$ is a sphere in $X$ let $L^{g^{\prime}}(S)=\left\{f \in L^{q^{\prime}}(S): \int_{S} f d \mu=0\right\}$. It follows that, if $f \in L_{0}^{g^{\prime}}(S)$, then

$$
a(x)=f(x)[\mu(S)]^{-1 / q}\|f\|_{L^{\prime}(S)}^{-1}
$$

is a $\left(1, q^{\prime}\right)$-atom supported in $S$. From this we also have $\|f\|_{1, q^{\prime}} \leqslant[\mu(S)]^{1 / q}$ $\|f\|_{L^{\prime}(S)}$. Hence, $L f$ is defined and

$$
|L f| \leqslant\|L\|[\mu(S)]^{1 / q}\|f\|_{L^{\prime}(S)}
$$

that is, $L$ is a bounded linear functional on $L g^{\prime}(S)$. Consequently, using the Hahn-Banach theorem and the Riesz representation theorem we conclude that there exists $l \in L^{q}(S)$ such that

$$
L f=\int_{S} f l d \mu
$$

for all $f \in L_{g}^{q^{\prime}}(S) .{ }^{31}$ The function $l$ is uniquely determined up to a constant; or, equivalently, if $\int_{S} f l d \mu=0$ for all $f \in L_{0}^{g^{\prime}}(S)$ it follows that $l$ is constant. To see this choose $h$ in $L^{q^{\prime}}(S)$; since $h-m_{S}(h) \in L^{q^{\prime}}(S)$ we have

$$
0=\int_{S} l\left(h-m_{S}(h)\right) d \mu=\int_{S} h\left(l-m_{S}(l)\right) d \mu .
$$

Since this equality holds for all $h \in L^{q^{\prime}}(S)$ it follows that $l(x)=m_{S}(l)$ for a.e. $x \in S$.

Let $\left\{S_{j}\right\}_{j=1}^{\infty}$ be an increasing sequence of spheres converging to $X$. We obtain a function $l_{j}=l$ satisfying (3.18) for each $S_{j}$ and, from the above argument, we see that the condition $\int_{S_{1}} l_{j} d \mu=0$ gives us a function $l$ for which (3.18) holds for any sphere $S$. In particular, if $a$ is a $\left(1, q^{\prime}\right)$-atom supported in $S$ we have

$$
\|L\| \geqslant|L a|=\left|\int_{S} l a d \mu\right|=\left|\int_{S}\left[l-m_{S}(l)\right] a d \mu\right| .
$$

If $f$ is supported in $S$ and has $L^{q^{\prime}}$ norm 1 then $a=2^{-1}[\mu(S)]^{-1 / q}\left(f-m_{S}(f)\right)$ is a $\left(1, q^{\prime}\right)$-atom. Using this atom in the last expression and using the fact that $l-m_{S}(l)$ has mean 0 on $S$ we obtain

$$
\left|\int_{S} f\left[l-m_{S}(l)\right] \frac{d \mu}{[\mu(S)]^{1 / q}}\right| \leqslant 2\|L\| .
$$

Taking the supremum of the left side over all $f$ supported in $S$ such that $\|f\|_{q^{\prime}}=1$ we obtain

$$
\left[\int_{S}\left|l-m_{S}(l)\right|^{q} \frac{d \mu}{\mu(S)}\right]^{1 / q} \leqslant 2\|L\| .
$$

\footnotetext{
${ }^{31}$ In order to apply the Riesz representation theorem we must assume $q^{\prime}<\infty$. This is not a restriction, however, since, by Theorem $\mathrm{A}, H^{1, \infty}=H^{1, r}$ for $1<r<\infty$.
} 
This shows that $l \in B M O_{q}$ with $\mathfrak{R}_{q}(l) \leqslant 2\|L\|$. In case $\mu(X)=1$ we also must have $\left|\int_{X} l d \mu\right|=\left|L \chi_{X}\right| \leqslant 1$. Thus, in general

$$
\|l\|_{B M O}^{(q)} \leqslant 3\|L\| .
$$

The characterization of the dual of $H^{p}$ for $p<1$ is simpler to obtain. Using Theorem A we see that we can restrict our attention to $H^{p, 1}$. Let $\alpha=1 / p-1$ and suppose $l \in \mathfrak{L}_{\alpha}$. Recall that the elements, $h$, of $H^{p, 1}$ are, by definition, continuous linear functionals in $\mathfrak{L}_{\alpha}^{*}$. Let us write $\langle h, l\rangle$ to denote the value of the linear functional $h$ at $l \in \mathfrak{L}_{\alpha}$. Then the mapping $L_{l}: h \rightarrow\langle h, l\rangle$ is a welldefined linear functional on $H^{\alpha, 1}$. If $h=\sum \alpha_{j} a_{j}$ is an atomic representation of $h$ in terms of $(p, 1)$-atoms we then have (see the estimates preceding (2.3))

$$
|\langle h, l\rangle|=\left|\sum \alpha_{j} \int l a_{j} d \mu\right| \leqslant\|l\|^{(\alpha)} \Sigma\left|\alpha_{j}\right| \leqslant\|l\|^{(\alpha)}\left(\sum\left|\alpha_{j}\right|^{p}\right)^{1 / p} .
$$

We see, therefore, that

$$
\left|L_{l} h\right| \leqslant\|l\|^{(\alpha)}\|h\|_{p, 1}^{1 / p} .
$$

Now suppose $L$ is a bounded ${ }^{32}$ linear functional on $H^{p, 1}$. As was the case when $p=1$ we can show the existence of a function $l$, bounded on each sphere, such that $L a=\int l a d \mu$ for each $(p, 1)$-atom $a$ (we use the same argument as before except that now $q^{\prime}=1$ and $q=\infty$ ). In particular, if $f$ is supported in a sphere $S$ and $\|f\|_{1}=1$ then $(1 / 2)[\mu(S)]^{1-1 / p}\left(f-m_{S}(f)\right)=a$ is a $(p, 1)$-atom and, thus,

$$
\|L\| \geqslant|L a|=\left|\int_{S}\left[l-m_{S}(l)\right] a d \mu\right|=2^{-1}[\mu(S)]^{1 / p-1}\left|\int_{S}\left[l-m_{S}(l)\right] f d \mu\right| .
$$

Taking the supremum over all such $f$ we obtain

$$
\left\|\chi_{S}\left(l-m_{S}(l)\right)\right\|_{\infty} \leqslant 2\|L\|[\mu(S)]^{\alpha} .
$$

It now follows from a straightforward argument that there exists $l_{0}=l$ a.e. for which

$$
\left|l_{0}(x)-l_{0}(y)\right| \leqslant 4\|L\|[\mu(S)]^{\alpha},
$$

where $S$ is any sphere containing $x$ and $y$. We then have

$$
\left\|l_{0}\right\|^{\alpha} \leqslant 5\|L\|
$$

whether the measure of $X$ is finite or infinite.

This establishes Theorem B. Inequalities (3.17), (3.18), (3.19) and (3.20) show that the norms of the functions representing the linear functionals are equivalent to the norms of the linear functionals.

In the argument preceding (3.19) we showed that $|\langle h, l\rangle| \leqslant\|l\|^{(\alpha)} \Sigma\left|\alpha_{j}\right|$, for

32 That is, there exists $\|L\|<\infty$ such that $|L h| \leqslant\|L\|\|h\|_{p, 1}^{1 / p}$. The space $H^{p, 1}$ is not a normed linear space, but it is obvious (by the usual arguments) that this notion of boundedness is equivalent to the continuity of $L$. 
$h \in H^{p, 1}$. In view of this inequality it is not surprising that the space $B_{p} \subset \mathfrak{L}_{\alpha}^{*}$ consisting of linear functionals of the form $h=\sum \alpha_{j} a_{j}$, where $\sum\left|\alpha_{j}\right|<\infty$ and $a_{j}$ are $(p, 1)$ - (or $(p, \infty)$ ) ) atoms, has a dual that is also characterized by $\mathfrak{L}_{\alpha}$ (with the norm $\|h\|^{(p)}$ taken as the infimum over all sums $\sum\left|\alpha_{j}\right|$, where $h=\sum \alpha_{j} a_{j}, B_{p}$ is a Banach space). In the classical case $X=T, B_{p}$ can easily be identified with the space of distributions $h$ whose Poisson integral $u_{h}(z)$ $=u_{h}(x+i y)$ satisfies

$$
\|h\|_{p}=\iint_{|z|<1}\left|u_{h}(z)\right|(1-|z|)^{1 / p-2} d x d y<\infty ;
$$

moreover, the norm $\|h\|_{p}$ is equivalent to the infimum of all sums $\Sigma\left|\alpha_{j}\right|$ arising from all the representations $h=\sum \alpha_{j} a_{j}$. From our point of view these spaces are not useful since the intermediate interpolation spaces between $L^{2}$ and $B_{p}$ are not $L^{p_{0}}(T)$ spaces when $p_{0}<1$ (in fact, they are spaces of harmonic functions in $L^{p_{0}}\left[(1-|z|)^{1 / p_{0}-2} d x d y\right]$.

Let us now pass to the proof of Theorem $\mathrm{D}$. We use the notation introduced in $\S 2$ : in particular, $B$ is a sublinear operator of weak-types $\left(H^{p_{1}}, p_{1}\right)$ and $\left(p_{2}, p_{2}\right)$ where $0<p_{1} \leqslant 1 \leqslant p_{2} \leqslant \infty, p_{1}<p_{2}$. Let us first suppose $1<p$, where $1 / p=t / p_{1}+(1-t) / p_{2}(0<t<1)$. Choose $q$ so that $1<q<p$ and let $\mathfrak{M}_{q}$ be the maximal function operator introduced just before inequality (3.7). Let $f \in L^{p}(X)$ and, for $\alpha>0$,

$$
0^{\alpha}=\left\{x \in X: \stackrel{\text { ). }}{\left.\left(\mathfrak{M}_{q} f\right)(x)>\alpha\right\} .}\right.
$$

As was the case in the proof of Theorem A we obtain a Calderon-Zygmund decomposition of $f$ from a Whitney covering of $0^{\alpha}$ by balls $S_{j}$ satisfying (i), (ii) and (iii) in (3.2) with $C=2 K$ :

$$
f=g_{\alpha}+h_{\alpha}
$$

where $g_{\alpha}(x)=g(x)=f(x)$ if $x \notin 0^{\alpha}, g_{\alpha}(x)=g(x)=\sum_{j} m_{S_{j}}\left(\eta_{j} f\right) \chi_{j}(x)$ when $x \in 0^{\alpha}, h_{j}=f \eta_{j}-m_{S_{j}}\left(\eta_{j} f\right) \chi_{j}$ and $h_{\alpha}=h=\sum_{j} h_{j}$. We then have

$$
\left(\frac{1}{\mu\left(S_{j}\right)} \int_{S_{j}}\left|h_{j}\right|^{q} d \mu\right)^{1 / q} \leqslant c \alpha
$$

(see the proof of induction hypothesis (VII) when $n=1$; the constant $c=2 k_{0}^{1 / q}$ depends only on $X$ ). It follows, therefore, that

$$
a_{j}=h_{j} / c \alpha\left[\mu\left(S_{j}\right)\right]^{1 / p_{1}}
$$

is a $\left(p_{1}, q\right)$-atom. Thus, $h=c \alpha \sum_{j}\left[\mu\left(S_{j}\right)\right]^{1 / p_{1}} a_{j} \in H^{p_{1}}\left(=H^{p_{1}, q}\right)$ with norm $\|h\|_{p_{1}} \sim\|h\|_{p_{1}, q}$ not exceeding

$$
(c \alpha)^{p_{1}} \sum_{j} \mu\left(S_{j}\right) \leqslant M(c \alpha)^{p_{1}} \mu\left(0^{\alpha}\right) .
$$

Let us first suppose $p_{2}<\infty$. Then, the weak-type hypotheses imply 


$$
\begin{aligned}
p^{-1}\|B f\|_{p}^{p}= & \int_{0}^{\infty} \alpha^{p-1} \nu(\{y \in Y:|(B f)(y)|>\alpha\}) d \alpha \\
\leqslant & \int_{0}^{\infty} \alpha^{p-1}\left[\nu\left(\left\{y \in Y:\left|\left(B g_{\alpha}\right)(y)\right|>\alpha / 2\right\}\right)\right. \\
& \left.\quad+\nu\left(\left\{y \in Y:\left|\left(B h_{\alpha}\right)(y)\right|>\alpha / 2\right\}\right)\right] d \alpha \\
\leqslant & \int_{0}^{\infty} \alpha^{p-1}\left(\frac{2 M_{2}\left\|g_{\alpha}\right\|_{p_{2}}}{\alpha}\right)^{p_{2}} d \alpha+\int_{0}^{\infty} \alpha^{p-1}\left(\frac{2 M_{1}}{\alpha}\right)^{p_{1}}\left\|h_{\alpha}\right\|_{p_{1}, q} d \alpha .
\end{aligned}
$$

In order to estimate the second integral we make use of (3.22) and (3.8) in order to dominate it by

$$
M\left(2 c M_{1}\right)^{p_{1}} \int_{0}^{\infty} \alpha^{p-1} \mu\left(0^{\alpha}\right) d \alpha=p^{-1} M\left(2 c M_{1}\right)^{p_{1}}\left\|\mathfrak{M}_{q} f\right\|_{p}^{p} \leqslant(\text { const })\|f\|_{p}^{p} .
$$

In order to estimate the first integral we write

$$
\begin{aligned}
& \int_{0}^{\infty} \alpha^{p-p_{2}-2}\left\{\int_{X}\left|g_{\alpha}(x)\right|^{p_{2}} d \mu(x)\right\} d \alpha \\
& \quad=\int_{0}^{\infty} \alpha^{p-p_{2}-2}\left\{\int_{0^{\alpha}}+\int_{X-0^{\alpha}}|g(x)|^{p_{2}} d \mu(x)\right\} d \alpha .
\end{aligned}
$$

But, as in the proof of Theorem A, we can show that $|g(x)| \leqslant c \alpha$ for all $x \in X$; thus,

$$
\begin{aligned}
\int_{0}^{\infty} \alpha^{p-p_{2}-2}\left\{\int_{0^{\alpha}}|g(x)|^{p_{2}} d \mu(x)\right\} d \alpha & \leqslant \text { (const) } \int_{0}^{\infty} \alpha^{p-1} \mu\left(0^{\alpha}\right) d \alpha \\
& =\text { (const) }\left\|\mathbb{M}_{q} f\right\|_{p}^{p} \leqslant(\text { const })\|f\|_{p}^{p} .
\end{aligned}
$$

Moreover,

$$
\begin{aligned}
\int_{0}^{\infty} & \alpha^{p-p_{2}-1}\left\{\int_{X-0^{\alpha}}|g(x)|^{p_{2}} d \mu(x)\right\} d \alpha \\
& =\int_{0}^{\infty} \alpha^{p-p_{2}-1} d \alpha \int_{\left(\mathfrak{M}_{q} f\right)(x) \leqslant \alpha}|f(x)|^{p_{2}} d \mu(x) \\
& =\int_{X}|f(x)|^{p_{2}}\left\{\int_{\left(\mathfrak{M}_{q} f\right)(x)}^{\infty} \alpha^{p-p_{2}-1} d \alpha\right\} d \mu(x) \\
& \leqslant \int_{X}|f(x)|^{p_{2}}\left\{\int_{|f(x)|} \alpha^{p-p_{2}-1} d \alpha\right\} d \mu(x)=\frac{1}{p_{2}-p} \int_{X}|f(x)|^{p} d \mu(x) .
\end{aligned}
$$

Collecting all these estimates we obtain the desired inequality $\|B f\|_{p} \leqslant$ (const) - $\|f\|_{p}$ and it is clear that the constant in this inequality depends on $M_{1}, M_{2}$, $p_{1}, p_{2}$ and $t$, but not on $f \in L^{p}(X)$. If $p_{2}=\infty$ the situation is simpler since $\left\|B g_{\alpha}\right\|_{\infty} \leqslant M_{2}\left\|g_{\alpha}\right\|_{\infty} \leqslant c M_{2} \alpha$ and, consequently, $\left\{y \in Y:\left|\left(B g_{\alpha}\right)(y)\right|\right.$ $\left.>c M_{2} \alpha\right\}$ is empty. Thus, 


$$
\begin{aligned}
\int_{0}^{\infty} \beta^{p-1} \nu(\{y \in Y:|(B f)(y)|>\beta\}) d \beta \\
\leqslant\left(2 c M_{2}\right)^{p} \int_{0}^{\infty} \alpha^{p-1}\left[\nu\left(\left\{y:\left|\left(B g_{\alpha}\right)(y)\right|>c M_{2} \alpha\right\}\right)\right. \\
\left.\quad+\nu\left(\left\{y:\left|\left(B h_{\alpha}\right)(y)\right|>c M_{2} \alpha\right\}\right)\right] d \alpha \\
=\left(2 c M_{2}\right)^{p} \int_{0}^{\infty} \alpha^{p-1} \nu\left(\left\{y:\left|\left(B h_{\alpha}\right)(y)\right|>c M_{2} \alpha\right\}\right) d \alpha .
\end{aligned}
$$

Now we can use the assumed weak-type $\left(H^{p_{1}}, p_{1}\right)$ assumption and proceed with an argument similar to that used above to obtain the $L^{p}$-boundedness of $B$.

The proof of the theorem for the case $p \leqslant 1$ is simpler. It suffices to estimate $\|B a\|_{p}$ when $a$ is a $(p, \infty)$-atom. When this is the case it is clear that $a=[\mu(S)]^{1 / p_{1}-1 / p} b(S$ is a supporting sphere for $a)$, where $b$ is a $\left(p_{1}, p_{2}\right)$-atom. Thus $\|a\|_{H^{p_{1}}} \leqslant[\mu(S)]^{1-1 / p_{1}}$. Using this estimate and the weak-type hypotheses we then have, putting $\sigma=[\mu(S)]^{-1 / p}$,

$$
\begin{aligned}
p^{-1}\|B a\|_{p}^{p} & =\int_{0}^{\infty} \alpha^{p-1} \nu(\{y:|(B a)(y)|>\alpha\}) d \alpha \\
& \leqslant \int_{0}^{\sigma} \alpha^{p-1}\left(\frac{M_{1}}{\alpha}\right)^{p_{1}} \sigma^{p_{1}-p} d \alpha+\int_{\sigma}^{\infty} \alpha^{p-1}\left(\frac{M_{2}}{\alpha}\right)^{p_{2}} \sigma^{p_{2}-p} d \alpha \\
& =M_{1}^{p} /\left(p-p_{1}\right)+M_{2}^{p} /\left(p_{2}-p\right) .
\end{aligned}
$$

The conclusion of Theorem $\mathrm{D}$ now follows easily from this.

The proof of Theorem E, when $p_{0}<p \leqslant 1$, is an adaptation to spaces of homogeneous type of the argument given in [58] which dealt with the classical $H^{p}$ spaces. When $1<p \leqslant p_{1}$ one can use an extension of the proof given by Fefferman and Stein in [29]. We shall not give a detailed proof of Theorem E (it is written up in [45]); rather, we shall indicate what is needed in order to extend these arguments to our situation.

Let us first consider the case $1<p \leqslant p_{1}$. Here, as in [29], the theorem is obtained by showing that the adjoint operator $B_{t}^{*}$ maps $L^{p^{\prime}}$ boundedly into $L^{p^{\prime}}$. In order to achieve this one makes use of the just established duality result for $H^{1}$ in order to convert boundedness from $H^{1}$ to $L^{1}$ into boundedness from $L^{\infty}$ to $B M O$. By employing a maximal operator that is particularly well adapted to BMO functions one can then reduce the problem to a classical interpolation theorem involving only $L^{p}$-spaces. This maximal operator was introduced for this purpose by Fefferman and Stein [29] when $X=\mathbf{R}^{n}$. Its definition and basic properties extend naturally to spaces of homogeneous type: Given a locally integrable function $f$ we let

$$
f^{\sharp}(x)=\sup \left\{\frac{1}{\mu(S)} \int_{S}\left|f(y)-m_{S}(f)\right| d \mu(y): x \in S\right\}
$$

(as usual, $S$ denotes a ball in $X$ ). Clearly $f^{\#} \leqslant 2 \mathfrak{M} f$ and $\left\|f^{\sharp}\right\|_{\infty} \leqslant\|f\|_{B M O}$. A basic property of this maximal operator is that, for $1 \leqslant p<\infty$, it dominates the Hardy-Littlewood maximal operator. When $\mu(X)=\infty$, in fact, we have $\left\|f^{*}\right\|_{p} \leqslant A_{p}\left\|f^{\#}\right\|_{p}$. The precise general statement of this result and its use for the interpolation result are found in [45]. 
When $p_{0}<p \leqslant 1$ this interpolation result was obtained independently by Calderón and Torchinski in the case dealing with $\mathbf{R}^{n}$ endowed with the distance of example (2) of [3].

4. Further observations. We have seen how $H^{1}$ can be used effectively as a substitute for $L^{1}$. It is natural to suppose that the space $B M O$ plays the same role vis-a-vis $L^{\infty}$. The proof of Theorem $\mathrm{E}$ which we have just described furnishes us an example of this situation. In fact, many results can be obtained efficiently by making use of $B M O$. This point is fully exploited in the work of Fefferman and Stein [29]. We shall see further evidence of this situation after having made some comments concerning the functional analysis properties of $H^{1}(X)$ and $B M O(X)$. Before doing this, however, we would like to express, again, our gratitude to $Y$. Meyer since many of the observations we shall make are either due to him or took place when discussing this work with him.

It follows easily from property (3.4) that if $X$ is complete with respect to the quasi-distance then it is locally compact (this was pointed out to us by $\mathbf{R}$. Levy). Since this is the situation in all the examples we have given of spaces of homogeneous type, we shall assume henceforth that $X$ is locally compact (or complete). It turns out that $H^{1}$ is one of the few examples of a separable, nonreflexive Banach space which is a dual space. In fact, let $V M O^{33}$ denote the closure in the $B M O$ norm of the space $C_{0}(X)$ of continuous functions with compact support. One can then prove the following result:

THEOREM (4.1). $H^{1}$ is the dual of VMO. More precisely, each continuous linear functional on $V M O$ has the form $\langle f, v\rangle=\int_{X} f v d \mu$ for all $v \in C_{0}$, where $f$ $\in H^{1}$, and $\|f\|_{H^{1}}$ is equivalent to the linear functional norm.

This result is a generalization of the theorem of F. and M. Riesz (see Sarason [52] in this connection). It shows that any Borel measure $v$ satisfying, for $v \in C_{0}(X)$,

$$
\left|\int v d v\right| \leqslant c\|v\|_{B M O}
$$

must be absolutely continuous with Radon-Nikodým derivative in $H^{1}$

Theorem (4.1) is a consequence of a general result in functional analysis ana a lemma that will be proved below. The functional analysis result (see Exercise 41 on p. 439 of [22]) is the following: Let $X$ be a locally convex linear topological space and $T$ a linear subspace of $X^{*}$. Then $T$ is $X$-dense in $X^{*}$ if and only if $T$ is a total set of functionals on $X$. The lemma we need is:

Lemma (4.2). Suppose $\left\|f_{k}\right\|_{H^{1}} \leqslant 1, k=1,2, \ldots$, then there exists $f \in H^{1}$ and a subsequence $\left(f_{k_{j}}\right)$ such that

$$
\lim _{j \rightarrow \infty} \int f_{k_{j}} v d \mu=\int f v d \mu
$$

for all $v \in C_{0}$.

33 These initials stand for "vanishing mean oscillation". A space VMO was introduced in the classical setting by Sarason [52]. It is somewhat different from ours; we adopted a modified version for which (4.1) holds. 
In our case $X=V M O$ and $T=H^{1}$. The fact that $T$ is a subspace of $X^{*}$ follows from the case $p=1$ of Theorem B (since $X$ is a subspace of $B M O$ ). In particular, if $\langle f, v\rangle=0$ for all $f \in H^{1}=T$ then $v$ must be the zero element of $V M O(\subset B M O)$. Thus, $T$ is total. By the above stated functional analysis result, it follows that $T$ is (weak*) dense in $X^{*}$. Since the spaces we are dealing with are separable this is equivalent to the statement: if $x^{*} \in X^{*}$ then we can find a sequence $\left\{f_{k}\right\}$ in $T\left(=H^{1}\right)$ such that $\left\langle f_{k}, v\right\rangle \rightarrow\left\langle x^{*}, v\right\rangle$ for all $v$ $\in X(=V M O)$. By the Banach-Steinhaus theorem we can conclude that the sequence of norms $\left\|f_{k}\right\|_{H^{1}}$ is bounded (this requires an elementary, but somewhat technical, argument showing that the supremum of $\left|\int \nu d \mu\right|$ over all $v \in C_{0}$ with $\|v\|_{B M O} \leqslant 1$ defines a norm that is equivalent to $\|f\|_{H^{1}}$ whenever $f \in H^{1}$. The principal step in this argument involves showing that $\left\|b_{\varepsilon}\right\|_{B M O}$ $\leqslant c\|b\|_{B M O}$ where $\left.b_{\varepsilon}(x)=\left[1 / \mu\left(S_{\varepsilon}(x)\right)\right] \int_{S_{\varepsilon}(x)} b(y) d \mu(y)\right)$. We can now invoke Lemma (4.2) to obtain a subsequence $\left\{f_{k_{j}}\right\}$ and an $f \in H^{1}$ such that

$$
\left\langle x^{*}, v\right\rangle=\lim _{j \rightarrow \infty}\left\langle f_{k_{j}}, v\right\rangle=\lim _{j \rightarrow \infty} \int f_{k_{j}} v d \mu=\int f v d \mu
$$

for all $v \in C_{0}$. From this it follows that the linear functional $x^{*}$ is represented by $f$ and we obtain Theorem (4.1).

In order to establish (4.2) we will need two lemmas. The first follows from a simple diagonalization argument:

Lemma (4.3). Suppose $\lambda_{j}^{k} \geqslant 0, j, k=1,2, \ldots$, satisfies $\sum_{j=1}^{\infty} \lambda_{j}^{k} \leqslant 1$ for each $k=1,2, \ldots$, then there exists an increasing sequence of natural numbers, $k_{1}<k_{2}<\cdots<k_{n}<\cdots$ such that $\lim _{n \rightarrow \infty} \lambda_{j}^{k_{n}}=\lambda_{j}$ for each $j$ and $\sum_{j=1}^{\infty} \lambda_{j}$ $\leqslant 1$.

The second lemma is of a geometric nature. For simplicity we consider the case where the spheres of radius $r$ have measure $r$ (and $\mu(X)=\infty$ ). By an "atom" we shall mean a $(1, \infty)$-atom.

Lemma (4.4). Suppose $n$ is an integer. Then $X$ is the union of balls $S_{i}^{n}, i=1$, $2, \ldots$, of measure $\beta^{n}$, where $\beta=\beta(X)>1$, such that no point belongs to more than $N$ of these balls $(N$ is independent of $n)$ and each $f \in H^{1}$ has the representation

$$
f=\sum_{i=1}^{\infty} \sum_{n=-\infty}^{\infty} \lambda_{i}^{n} a_{i}^{n}
$$

where $a_{i}^{n}$ is an atom supported in $S_{i}^{n}$ and $\sum_{i, n}\left|\lambda_{i}^{n}\right| \leqslant c\|f\|_{H^{1}}$.

We shall sketch the proof of this lemma. If $B$ is any sphere and $\bar{B}$ is the smallest sphere containing all those balls intersecting $B$ and having the same radius as $B$ then the ratio $\mu(\bar{B}) / \mu(B)$ is bounded independently of $B$. Let $\beta$ be such a bound. An argument similar to the one used to establish (3.2) shows that $X$ can be covered with balls $B_{1}^{n}, B_{2}^{n}, \ldots, B_{j}^{n}, \ldots$ of measure $\beta^{n}$ that are almost disjoint. Moreover, this can be done in such a way that $\left\{b_{j}^{n}\right\}$ is a sequence of mutually disjoint balls and, for each $j, b_{j}^{n}$ has the same center as $B_{j}^{n}$ and a radius a fixed multiple (depending only on $A$ and $K$ ) of the radius of $B_{j}^{n}$. Let $f=\sum \lambda_{k} a_{k}$. Without loss of generality we can assume each $a_{k}$ is an 
atom supported by a sphere of measure $\beta^{n}$ for some $n$ (the infimum of $\Sigma\left|\lambda_{j}\right|$ over all such decompositions differs from $\|f\|_{H^{\prime}}$ by a constant multiple that depends on $\beta$ but not on $f$ ). We make our choice of the representation of $f$ so that $\sum\left|\lambda_{j}\right| \leqslant c\|f\|_{H^{1}}(c$ independent of $f)$. Let $n=0$ and consider all atoms $a_{j}$ whose supporting spheres have measure 1 and intersect $B_{1}^{0}$. The sum of terms $\lambda_{k} a_{k}$ involving these atoms is supported in a sphere $S_{1}^{1}$ of measure $\beta$ and can be written in the form $\lambda_{1}^{1} a_{1}^{1}$, where $a_{1,1}$ is an atom supported in $S_{1}^{1}$. Now consider the remaining atoms whose supporting sphere has measure 1 . Collect those intersecting $B_{2}^{0}$ and, similarly, obtain $\lambda_{2}^{1} a_{2}^{1}$. Continue inductively until all atoms supported by spheres of measure 1 are exhausted. Now let $n=-1$ and do the same for atoms whose supporting spheres have measure $\beta^{-1}$ and intersect $B_{1}^{-1}$. We obtain $\lambda_{1}^{0} a_{1}^{0}, \lambda_{2}^{0} a_{2}^{0}, \ldots$ Next, consider $n=1$, etc. Observe that, by definition, $\lambda_{j}^{n} a_{j}^{n}$ is a sum $\sum \lambda_{k} a_{k}=\alpha_{j}^{n}$ over only indices $k$ such that $a_{k}$ has a supporting sphere of measure $\beta^{n-1}$ meeting $B_{j}^{n-1}$. Thus, $\left\|\alpha_{j}^{n}\right\|_{\infty}$ $\leqslant \sum\left|\lambda_{k}\right| \beta^{1-n}$ (the sum is over this set of indices). Hence, we let $\lambda_{j}^{n}$ $=\left\|\alpha_{j}^{n}\right\|_{\infty} \mu\left(S_{j}^{n}\right) \leqslant \beta \sum\left|\lambda_{k}\right|$. By our construction, these indices occur only once. Thus, we obtain the last inequality in (4.4). The almost disjointness of the sequence $S_{1}^{n}, S_{2}^{n}, \ldots$ follows from the disjointness of the sequence $b_{1}^{n-1}, b_{2}^{n-1}, \ldots$ and the fact that the balls in each of the two sequences have the same radius.

We are now ready to prove Lemma (4.2). We write $f_{k}=\sum_{i, n} \lambda_{i}^{n}(k) a_{i}^{n}(k)$ as in Lemma (4.4). We can suppose that the coefficients $\lambda_{i}^{n}(k)$ are nonnegative and, applying Lemma (4.3) we can assume $\lim _{k \rightarrow \infty} \lambda_{i}^{n}(k)=\lambda_{i}^{n}$ exists for each $(i, n)$ and $\sum_{i, n} \lambda_{i}^{n} \leqslant 2$. For each fixed $(i, n)$ the atoms $a_{i}^{n}(k)$ are supported by the sphere $S_{i}^{n}$ for all $k$ and are uniformly bounded. Thus, there exists a subsequence of $a_{i}^{n}(1), a_{i}^{n}(2), \ldots, a_{i}^{n}(k), \ldots$ converging weakly to a function $a_{i}^{n}$ (that is

$$
\int_{S_{i}^{n}} a_{i}^{n}\left(k_{j}\right) \varphi d \mu \rightarrow \int_{S_{i}^{n}} a_{i}^{n} \varphi d \mu
$$

for all $\left.\varphi \in L^{1}\left(S_{i}^{n}\right)\right)$. Applying, again, a diagonalization argument we can assume $a_{i}^{n}(k) \rightarrow a_{i}^{n}$ as $k \rightarrow \infty$ for all $(i, n)$. We let $f=\sum \lambda_{i}^{n} a_{i}^{n}$. It is clear that $f \in H^{1}$. It remains to be shown that $\lim _{k \rightarrow \infty} \int f_{k} v d \mu=\int f v d \mu$ for all $v \in C_{0}$. We write

$$
\int f_{k} v d \mu=\left(\sum_{-N \leqslant n \leqslant N}+\sum_{n<-N}+\sum_{n>N}\right) \sum_{i} \lambda_{i}^{n}(k) \int a_{i}^{n}(k) v d \mu .
$$

The last sum is dominated by a constant multiple of $\beta^{-N} \sum_{n>N} \sum_{i} \lambda_{i}^{n}(k)\|v\|_{1}$ which is small provided $N$ is large. Also if $N$ is large we have $\left|v(x)-v\left(x_{i}^{n}\right)\right|$ $<\varepsilon$ for $x \in S_{i}^{n}$ if $x_{i}^{n}$ is the center of $S_{i}^{n}$ and $n<-N$. Thus, the second sum is dominated by

$$
\sum_{n<-N} \sum_{i} \lambda_{i}^{n}(k)\left|\int a_{i}^{n}(k)(x)\left\{v(x)-v\left(x_{i}^{n}\right)\right\} d \mu(x)\right| \leqslant \varepsilon .
$$

The first sum involves only a finite number of terms, independently of $k$ (here we use the almost disjointness, for each $n$, of the spheres $S_{i}^{n}$ and the fact that 
the compact support of $v$ can only meet a finite number of these spheres since their radii range only from $\beta^{-N}$ to $\beta^{N}$ ). Thus, the first sum tends to $\int\left(\sum_{-N \leqslant n \leqslant N} \sum_{i} \lambda_{i}^{n} a_{i}^{n}\right) v d \mu$ which, for the above reasons, is close to $\int f v d \mu$ if $N$ is large. This establishes (4.2) and, consequently, Theorem (4.1) is proved.

This theorem shows, in particular, that whenever $L^{1}(X)$ is not a dual space ${ }^{34}$ $H^{1}(X)$ is a basically different space. Another way to see that $H^{1}(X)$ differs from $L^{1}(X)$ is to exhibit an unbounded function in $B M O(X)$. For a wide class of spaces of homogeneous type we obtain a collection of such functions by letting $g=\log v^{*}$, where $v$ is a nonnegative locally finite measure such that

$$
v^{*}(x)=\sup _{x \in S} \frac{\nu(S)}{\mu(S)}<\infty
$$

for almost every $x$ (with respect to $\mu$ ). In particular, if $\nu$ is a point mass at $x_{0}$ then $\log m\left(x, x_{0}\right) \in B M O$ (here $m$ denotes the "measure distance" mentioned in footnote 20). These functions cannot be bounded unless $x$ has a finite number of points.

Let us recall, once again, that a most basic result in the theory of $H^{p}$ spaces is their characterization in terms of maximal averages (in the classical case we are referring to [2]; for the $n$-dimensional case see [29]). During the course of a conversation with $Y$. Meyer, the three of us observed that a recent proof of the duality result between $H^{1}$ and $B M O$, due to $\mathrm{L}$. Carleson [8] can be extended to the general setting of spaces of homogeneous type provided a certain geometric assumption is added. From this one can then obtain a "maximal function" characterization of $H^{1}$. The result of Carleson, in the general setting, can be formulated in the following way:

Let $\left\{k_{r}(x, y)\right\}, r>0$, be a family of nonnegative valued functions on $X \times X$ satisfying

(1) $k_{r}(x, y)=0$ if $d(x, y) \geqslant r$;

(2) there exists constants $c$ and $C$ such that for all $x, y \in X$

$$
k_{r}(x, x) \geqslant \frac{c}{\mu\left(B_{r}(x)\right)} \text { and } k_{r}(x, y) \leqslant \frac{C}{\mu\left(B_{r}(x)\right)} ;
$$

(3) $\int_{X} k_{r}(x, y) d \mu(y)=1$ for all $x \in X$;

(4) there exists an $\alpha>0$ such that for all $x_{1}, x_{2}, y \in X$

$$
\left|k_{r}\left(x_{1}, y\right)-k_{r}\left(x_{2}, y\right)\right| \leqslant \frac{C}{\mu\left(B_{r}(y)\right)}\left[\frac{d\left(x_{1}, x_{2}\right)}{r}\right]^{\alpha} .
$$

When $X=\mathbf{R}$ such a family is obtained by choosing a nonnegative "bellshaped" Lipschitz function $\varphi$ supported by the interval $(-1 / 2,1 / 2)$, having integral 1 and putting $k_{r}(x, y)=(1 / r) \varphi((x-y) / r)$. The more general geometric assumptions needed for constructing such a family can be stated in terms of the measure distance $m$ as follows: there exists $\varepsilon>0$ such that

\footnotetext{
34 This is, in fact, the usual situation. The space $l^{1}$ of absolutely summable sequences is exceptional: it is the dual of $c_{0}$, the space of sequences converging to 0 . The proof we have just given, among other things, shows that $H^{1}(X)$, in general, is similar to $l^{1}$.
} 


$$
\mu\left(B_{x} \cap B_{y}\right) \leqslant\left\{\frac{m(x, y)}{\mu\left(B_{x} \cup B_{y}\right)}\right\}^{\varepsilon} \mu\left(B_{x} \cup B_{y}\right)
$$

whenever $B_{x}\left(B_{y}\right)$ is a ball containing $x(y)$. If this condition is satisfied Carleson's duality proof is based on a characterization of BMO functions:

THEOREM (4.5). Suppose $\varphi \in B M O$ has support within a ball $B \subset X$ then there exists a constant $C=C(X)>0$, a measurable function $r(y)>0$ and two bounded functions $b, \psi$ such that

(a) $\varphi(x)=\int k_{r(y)}(x, y) b(y) d \mu(y)+\psi(x)$;

(b) $\|b\|_{\infty} \leqslant C\|\varphi\|_{B M O}$ and $\|\psi\|_{\infty} \leqslant C\|\varphi\|_{B M O}$;

(c) the supports of $b$ and $\psi$ are within $B$.

The maximal function of $f$, with respect to the family $\left\{k_{r}\right\}$ is defined by letting its value at $x \in X$ be

$$
f^{*}(x)=\sup _{r>0} \int k_{r}(x, y) f(y) d \mu(y) .
$$

Making use of (4.5) one can show that an integrable $f$ belongs to $H^{1}$ if and only if $f^{*} \in L^{1}(X)$.

It is not clear to us to what extent the above geometric condition is necessary for the validity of this characterization of $H^{1}$. Moreover, the original one-dimensional methods in [10] and their extension to $n$ dimensions by Latter are applicable in several of the examples of spaces of homogeneous type we have presented here; they yield a direct atomic decomposition of these "maximal function" $H^{p}$ spaces, even for some $p<1$. It would be of interest to clarify this situation further.

Before ending this presentation we would like to say a few words about an important class of Hardy spaces for which an "atomic" theory has not been developed. These are the $H^{p}$ spaces on the polydiscs, orginally introduced by Zygmund [64], and their extensions to Reinhardt regions and tubes (see [57] and [51]). It is reasonable to suppose that, in $\mathbf{R}^{n}$, these spaces are related to an atomic $H^{1}$ space generated by atoms whose support lies within rectangles with sides parallel to the axes and the cancellation properties occur along each axis. For example, a rectangular $(1, \infty)$-atom on $\mathbf{R}^{2}$ is a function supported in the rectangle $R$, satisfying $|a(x, y)| \leqslant 1 /|R|$, for all $(x, y) \in \mathbf{R}^{2}$ and

$$
\int_{-\infty}^{\infty} a(x, y) d y=0=\int_{-\infty}^{\infty} a(x, y) d x .
$$

These atoms generate a subspace $\mathfrak{S}^{1}$ of our "cubic" $H^{1}\left(\mathbf{R}^{2}\right)$. The latter is characterized by the Riesz transforms. It is easy to see that $\mathfrak{G}^{1}$ is invariant under the iterated Hilbert transforms. Carleson and Fefferman showed, however, that this property does not characterize $\mathfrak{S}^{1}$. Nevertheless, it may turn out to be another useful substitute for $L^{1}$. A natural question is to see if the interpolation theorems we have considered in this exposition are valid here. A positive result along these lines would certainly imply some interesting multiplier theorems. Another question is to see if there are "natural operators" characterizing $\mathfrak{S}^{1}$. 


\section{REFERENCES}

1. R. Boas, Isomorphism between $H^{p}$ and $L^{p}$, Amer. J. Math. 77 (1955), 655-656. MR 17, 1080.

2. D. L. Burkholder, R. F. Gundy and M. L. Silverstein, A maximal function characterization of the class $H^{p}$, Trans. Amer. Math. Soc. 157 (1971), 137-153. MR 43 \#527.

3. A. P. Calderón and A. Torchinsky, Spaces of distributions in $L^{p}\left(\mathbf{R}^{n}\right)$ (to appear).

4. A. P. Calderón and A. Zygmund, Singular integral operators and differential equations, Amer. J. Math. 79 (1959), 901-921. MR 20 \#196.

5. - On the existence of certain singular integrals, Acta Math. 88 (1952), 85-139. MR 14, 637.

6. On higher gradients of harmonic functions, Studia Math. 24 (1964), 211-226. MR 29 \#4903.

7. S. Campanato, Proprietà di hölderianità di alcune classi di funzioni, Ann. Scuola Norm. Sup. Pisa (3) 17 (1963), 175-188. MR 27 \#6119.

8. L. Carleson, Two remarks on $H^{1}$ and $B M O$, Analyse Harmonique d'Orsay 164 (1975), 1-11.

9. F. Carlson, Une inégalité, Ark. Mat. Astronom. Fys. 25B, no. 1, (1934), 1-5.

10. R. R. Coifman, A real variable characterization of $H^{p}$, Studia Math. 51 (1974), 269-274. MR 50 \# 10784.

11. Characterizations of Fourier transforms of Hardy spaces, Proc. Nat. Acad. Sci. U. S. A. 71 (1974), no. 10, 4133-4134.

12. R. R. Coifman and M. de Guzmán, Singular integrals and multipliers on homogeneous spaces, Rev. Un. Mat. Argentina 25 (1970/71), 137-143. MR 47 \#9180.

13. R. R. Coifman and C. Fefferman, Weighted norm inequalities for maximal functions and singular integrals, Studia Math. 51 (1974), 241-250. MR 50 \#10670.

14. R. R. Coifman, R. Rochberg and G. Weiss, Factorization theorems for Hardy spaces in several variables, Ann. of Math. 103 (1976), 611-635.

15. R. R. Coifman and G. Weiss, On subharmonicity inequalities involving solutions of generalized Cauchy-Riemann equations, Studia Math. 36 (1970), 77-83. MR 42 \#6265.

16. - Invariant systems of conjugate harmonic functions associated with compact Lie groups, Studia Math. 44 (1972), 301-308.

17. - Analyse harmonique non-commutative sur certains espaces homogenes, Lecture Notes in Math., vol. 242, Springer-Verlag, Berlin and New York, 1971.

18. - Théorèmes sur les multiplicateurs de Fourier sur $S U(2)$ et $\Sigma_{2}$, C. R. Acad. Sci.Paris Sér. A-B 271 (1970), pp. A928-A930. MR 42 \#6157.

19. - A multiplier theorem for $S U(2)$ and $\Sigma_{2}$, Rev. Un. Mat. Argentina 25 (1970), $145-166$.

20. W. Connett and A. Schwartz, A multiplier theorem for ultraspherical series, Studia Math. 51 (1974), 51-70. MR 50 \# 10674.

21. M. Cotlar, $A$ unified theory of Hilbert transforms and ergodic theorems, Rev. Mat. Cuyana 1 (1955), 105-167 (1956). MR 18, 893.

22. N. Dunford and J. Schwartz, Linear operators. I, Interscience, New York and London, 1964 (1st ed., 1958; MR 22 \# 8302).

23. P. L. Duren, Theory of $H^{p}$ spaces, Academic Press, New York, 1970. MR 42 \#3552.

24. P. L. Duren, B. W. Romberg and A. L. Shields, Linear functionals on $H^{p}$ spaces with $0<p<1$, J. Reine Angew. Math. 238 (1969), 32-60. MR 41 \#217.

25. A. Erdélyi et al., Higher transcendental functions, Vol. II, McGraw-Hill, New York, 1953. MR 15, 419; 34 \# 1565.

26. E. B. Fabes and N. M. Rivière, Singular integrals with mixed homogeneity, Studia Math. 27 (1966), 19-38. MR 35 \#683.

27. C. Fefferman, $H^{p}$ spaces, Harmonic Analysis (Studies in Math.,Vol. 13, M. Ash, Ed.), Math. Assoc. of Amer., 1976, 38-75.

28. Characterizations of bounded mean oscillation, Bull. Amer. Math. Soc. 77 (1971), 587-588. MR 43 \#6713.

29. C. Fefferman and E. M. Stein, $H^{p}$ spaces of several variables, Acta Math. 129 (1972), 137-193.

30. C. Fefferman, N. M. Rivière and Y. Sagher, Interpolation between $H^{p}$ spaces: The real method, Trans. Amer. Math. Soc. 191 (1974), 75-81. 
31. G. B. Folland and E. M. Stein, Estimates for the $\bar{\partial}_{b}$-complex and analysis on the Heisenberg group, Comm. Pure Appl. Math. 27 (1974), 429-522.

32. J. Garcia-Cuerva, Weighted $H^{P_{-}}$-spaces, Ph.D. Thesis, Washington Univ., Missouri, 1975.

33. G. H. Hardy, The mean value of the modules of an analytic function, Proc. London Math. Soc. (2) 14 (1915), 269-277.

34. C. Herz, Bounded mean oscillation and regulated martingales, Trans. Amer. Math. Soc. 193 (1974), 199-215. MR 50 \#5930.

35. K. Hoffman, Banach spaces of analytic functions, Prentice-Hall, Englewood Cliffs, N. J., 1962. MR $24 \# 2844$.

36. L. Hörmander, Estimates for translation invariant operators in $L^{p}$ spaces, Acta Math. 104 (1960), 93-140. MR 22 \#12389.

37. S. Igari, An extension of the interpolation theorem of Marcinkiewicz. II, Tôhoku Math. J. (2) 15 (1963), 343-358. MR 28 \#1431.

38. F. John and L. Nirenberg, On functions of bounded mean oscillation, Comm. Pure Appl. Math. 14 (1961), 415-426. MR 24 \#A1348.

39. B. F. Jones, Jr., A class of singular integrals, Amer. J. Math. 86 (1964), 441-462. MR 28 \#4308.

40. A. W. Knapp and E. M. Stein, Intertwining operators for semi-simple Lie groups, Ann. of Math. (2) 93 (1971), 489-578.

41. - Singular integrals and the principal series. I, Proc. Nat. Acad. Sci. U.S.A. 63 (1969), 281-284. MR 41 \#8588.

42. A. Korányi and S. Vági, Singular integrals on homogeneous spaces and some problems of classical analysis, Ann. Scuola Norm. Sup. Pisa 25 (1971), 575-648.

43. P. Krée, Distributions quasi homogènes. Généralisations des intégrals singulières et du calcul symbolique de Calderón-Zygmund, C. R. Acad. Sci. Paris 261 (1965), 2560-2563. MR 32 \#6211.

44. V. Krylov, Functions regular in the half-plane, Sbornik 6 (1939), 95-138. (Russian)

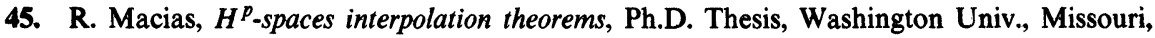
1975.

46. N. G. Meyers, Mean oscillation over cubes and Hölder continuity, Proc. Amer. Math. Soc. 15 (1964), 717-721. MR 29 \#5969.

47. B. Muckenhoupt and E. M. Stein, Classical expansions and their relation to conjugate harmonic functions, Trans. Amer. Math. Soc. 118 (1965), 17-92. MR 33 \#7779.

48. F. Riesz, Sur les valeurs moyennes du module des fonctions harmoniques et des fonctions analytiques, Acta Sci. Math. 1 (1922/23), 27-32.

49. N. M. Rivière, Singular integrals and multiplier operators, Ark. Mat. 9 (1971), no. 2 , 243-278.

50. L. P. Rothschild and E. M. Stein, Hypoelliptic differential operators and nilpotent groups (preprint).

51. W. Rudin, Function theory in polydiscs, Benjamin, New York, 1969. MR 41 \# 501.

52. D. Sarason, Functions of vanishing mean oscillation, Trans. Amer. Math. Soc. 207 (1975), 391-405.

53. E. M. Stein, Note on the class $L \log L$, Studia Math. 32 (1969), 305-310. MR 40 \#799.

54. - Classes $H^{p}$, multiplicateurs et fonctions de Littlewood-Paley, C. R. Acad. Sci. Paris

Sér. A-B 263 (1966), A716-A719, A780-A781; ibid. 264 (1967), A107-A108. MR 37 \#695a, b, c.

55. - Singular integrals and differentiability properties of functions, Princeton Univ. Press,

Princeton, N. J., 1970. MR 44 \# 7280.

56. - Boundary behavior of holomorphic functions of several complex variables, Math.

Notes, Princeton Univ. Press, Princeton, N. J., 1972.

57. E. M. Stein and G. Weiss, Introduction to Fourier analysis on Euclidean spaces, Princeton Univ. Press, Pinceton, N. J., 1971. MR 46 \#102.

58. - On the interpolation of analytic families of operators acting on $H^{P_{-}}$-spaces, Tôhoku Math. J. (2) 9 (1957), 318-339. MR 20 \# 1216.

59. - On the theory of harmonic functions of several variables. I, Acta Math. 103 (1960), 25-62. MR 22 \#12315.

60. - Generalization of the Cauchy-Riemann equations and representations of the rotation group, Amer. J. Math. 90 (1968), no. 1, 163-196. MR 36 \#6540. 
61. M. H. Taibleson, Fourier analysis on local fields, Math. Notes, Princeton Univ. Press, Princeton, N. J., 1975.

62. G. Weiss, Complex methods in harmonic analysis, Amer. Math. Monthly 77 (1970), no. 5, 465-474. MR 41 \# 5886.

63. - Harmonic analysis, Studies in real and complex analysis (Studies in Math., Vol. III), Math. Assoc. of Amer., Buffalo, N. Y.; Prentice-Hall, Englewood Cliffs, N. J., 1965, pp. 124-178. MR 32 \# 1080.

64. A. Zygmund, Trigonometric series, 2nd. rev. ed., Vols. I, II, Cambridge Univ. Press, New York, 1959. MR 21 \#6498.

Department of Mathematics, Washington University, St. Louis, Missouri 63130 